\section{Superconductivity in High Energy Particle Accelerators ${ }^{1}$ Peter Schmüser \\ Institut für Experimentalphysik Universität Hamburg}

\begin{abstract}
The basics of superconductivity are outlined with special emphasis on the features which are relevant for the application in magnets and radio frequency cavities for high energy particle accelerators. The special properties of superconducting accelerator magnets are described in detail: design principles, magnetic field calculations, magnetic forces, quench performance, persistent magnetization currents and eddy currents. The design principles and basic properties of superconducting cavities are explained as well as the observed performance limitations and the countermeasures. The ongoing research efforts towards maximum accelerating fields are addressed and the coupling of radio frequency power to the particle beam is treated.
\end{abstract}

\section{Introduction}

\subsection{Advantages and limitations of super- conductor technology in accelerators}

The vanishing electrical resistance of superconducting coils as well as their ability to provide magnetic fields far beyond those of saturated iron is the main motivation for using superconducting (sc) magnets in all new large proton, antiproton and heavy ion accelerators ${ }^{2}$. The first machine of this kind, the Tevatron [2] at the Fermi National Accelerator Laboratory (FNAL) near Chicago, USA, has been operating as a proton-antiproton collider for many years, featuring centre-of-mass energies of 1800 $\mathrm{GeV}$ with excellent luminosity. The successful dipole and quadrupole magnets developed at FNAL have strongly influenced the design of the superconducting magnets for the proton-electron collider HERA [3] at DESY, the Large Hadron Collider LHC [4] at CERN and the Relativistic Heavy Ion Collider RHIC [5] at Brookhaven. Superconductivity does not only open the way to much higher particle energies but at the same time leads to a substantial reduction of operating costs. In the normalconducting Super Proton Synchrotron SPS at CERN a power of $52 \mathrm{MW}$ is needed to excite the machine to an energy of $315 \mathrm{GeV}$ while at HERA a cryogenic plant with $6 \mathrm{MW}$ electrical power consumption is sufficient to provide the cooling of the superconducting magnets with a stored proton beam of $920 \mathrm{GeV}$. Hadron energies in

\footnotetext{
${ }^{1}$ Expanded version of a review article in Prog. Part. Nucl. Phys. 49 (2002) issue 1

${ }^{2}$ The parameters of high energy lepton and hadron colliders are summarized in [1].
}

the $\mathrm{TeV}$ regime are practically inaccessible with standard magnet technology. Another important application of superconducting materials is in the large experiments at hadron or lepton colliders where superconducting detector magnets are far superior to normal magnets.

In the case of accelerating cavities the advantage of superconductors is not at all that obvious. In fact three of the proposed linear electron positron colliders are based on copper acceleration structures (the 'Next Linear Collider' NLC [6] at Stanford, USA, the 'Japanese Linear Collider' JLC [7] at Tsukuba, Japan and the 'Compact Linear Collider' CLIC [8] at CERN, Switzerland) while only the international TESLA project $[9,10]$ uses sc niobium cavities. The traditional arguments against superconductor technology in linear colliders have been the low accelerating fields achieved in sc cavities and the high cost of cryogenic equipment. Superconducting cavities face a strong physical limitation: the microwave magnetic field must stay below the critical field of the superconductor. For the best superconductor for cavities, niobium, this corresponds to a maximum accelerating field of about $50 \mathrm{MV} / \mathrm{m}$ while normal-conducting cavities operating at high frequency (above $5 \mathrm{GHz}$ ) should in principle be able to reach $100 \mathrm{MV} / \mathrm{m}$ or more. In practice, however, sc cavities were often found to be limited at much lower fields of some $5 \mathrm{MV} / \mathrm{m}$ and hence were totally non-competitive for a linear collider. Great progress was achieved with the 340 five-cell cavities of the Continuous Electron Beam Accelerator Facility CEBAF [11] [11] at Jefferson Laboratory in Virginia, USA. These 1.5 $\mathrm{GHz}$ niobium cavities were developed at Cornell University and produced by industry. They exceeded the design gradient of $5 \mathrm{MV} / \mathrm{m}$ and achieved $8.4 \mathrm{MV} / \mathrm{m}$ after installation in the accelerator (in several specially prepared cavities even 15-20 MV/m were reached). Building upon the CEBAF experience the intensive $R \& D$ of the TESLA collaboration has succeeded in raising the accelerating field in multicell cavities to more than $25 \mathrm{MV} / \mathrm{m}$. There is a realistic chance to reach even $35 \mathrm{MV} / \mathrm{m}$, and to reduce substantially the cost for the cryogenic installation.

Superconducting magnets operated with direct current are free of energy dissipation, however, this is not the case in microwave cavities. The non-superconducting electrons (see sect. 2) experience forced oscillations in the time-varying magnetic field and dissipate power in the material. Although the resulting heat deposition is many orders of magnitude smaller than in normal cavities it constitutes a significant heat load on the refrigeration system. As a rule of thumb, $1 \mathrm{~W}$ of heat deposited at $2 \mathrm{~K}$ requires almost $1 \mathrm{~kW}$ of primary ac power in the refrigerator. There is now a worldwide consensus that the overall efficiency for converting primary electric power into beam power is about a factor two higher for a superconducting than for a normal-conducting linear collider with optimized parameters in either case [12]. Another definite advantage of a superconducting collider is the 
low resonance frequency of the cavities that can be chosen $(1.3 \mathrm{GHz}$ in TESLA). The longitudinal (transverse) wake fields generated by the ultrashort electron bunches upon passing the cavities scale with the second (third) power of the frequency and are hence much smaller in TESLA than in NLC $(f=11 \mathrm{GHz})$. The wake fields may have a negative impact on the beam emittance (the area occupied in phase space) and on the luminosity of the collider.

\subsection{Characteristic properties of super- conducting accelerator magnets}

Superconducting magnets have a number of properties which are not found in normal magnets and must be taken into consideration when designing the dipoles, quadrupoles and correction magnets of a superconducting accelerator.

Field quality. A high field quality is needed in the magnets of a hadron storage ring if one wants to store an intense particle beam for many hours. The relative deviation from the ideal dipole or quadrupole field must not exceed a few parts in $10^{4}$. This poses no particular problem with normal magnets whose field distribution is determined by accurately shaped iron yokes. In a superconducting coil, however, the field pattern is governed by the arrangement of the current conductors and a precise coil geometry is of utmost importance. The positional accuracy required for the conductors in the coil is in the $20 \mu \mathrm{m}$ range. This precision must be maintained in spite of the huge Lorentz forces acting on the current conductors: the two halves of a dipole coil repel each other with a typical force of $10^{6} \mathrm{~N}$ (100 tons) per metre length at a field of 5 Tesla. The coils are confined by strong clamps which take up the Lorentz forces and define the exact geometry.

Quenches and magnet protection. A quench is the transition from the superconducting to the normal state. Such a transition will invariably occur if any of the three parameters: temperature, magnetic field or current density exceeds a critical value. The origin may be heating by beam losses or by a conductor motion under the influence of Lorentz forces. At high coil currents a motion of a few $\mu \mathrm{m}$ may be sufficient since only a tiny energy deposition, in the order of $1 \mathrm{~mJ} / \mathrm{g}$, is needed to heat the conductor beyond the critical temperature. The reason for this extreme sensitivity is the very low heat capacity of metals at low temperature $\left(C \propto T^{3}\right.$ in the limit $T \rightarrow 0)$. Liquid helium is the only substance with an appreciable heat capacity in the $2-4 \mathrm{~K}$ range. A good thermal contact between the superconducting cable and the helium coolant is essential for the stability of the coil.

If a quench happens in a large dipole the current in the coil must be reduced to zero in a short time interval (typically in less than a second at $5 \mathrm{~T}$ ) to avoid overheating and possible destruction of the normal conducting part of the coil. A fast and reliable quench detection and mag- net protection system is one of the most important safety features of a superconducting accelerator. It is equally important to construct the magnets in such a way that they have a high inherent stability against quenches.

Persistent magnetization currents. The advantage of superconducting coils turns into a drawback at low fields. Any field variation induces bipolar magnetization currents in the superconductor which - in contrast to the eddy currents in conventional electromagnets - do not decay exponentially but flow indefinitely. These 'persistent currents' generate field distortions which may become intolerably large at low excitation. A well-known example is the sextupole component measured in all superconducting dipoles. In a machine with very low injection energy like HERA even multipoles of higher order play a role and require a compensation by correction coils. The persistent currents are not exactly constant but exhibit a slow, nearly logarithmic time dependence. The current in the correction coils has to be adjusted to compensate the drift.

\subsection{Characteristic properties of super- conducting cavities}

The fundamental advantage of superconducting niobium cavities is the extremely low surface resistance of about $10 \mathrm{n} \Omega$ at 2 Kelvin as compared to several $\mathrm{m} \Omega$ in copper cavities. The quality factor $Q_{0}(2 \pi$ times the ratio of stored energy to energy loss per cycle) is inversely proportional to the surface resistance and may exceed $10^{10}$. Only a tiny fraction of the incident radio frequency (rf) power is dissipated in the cavity walls, the lion's share is transferred to the beam. The physical limitation of a sc resonator is given by the requirement that the rf magnetic field at the inner surface has to stay below the critical field of the superconductor (about $200 \mathrm{mT}$ for niobium), corresponding to an accelerating field of $E_{a c c}=50 \mathrm{MV} / \mathrm{m}$. In principle the quality factor should stay constant when approaching this fundamental superconductor limit but in practice the curve $Q_{0}=Q_{0}\left(E_{a c c}\right)$ ends at considerably lower values, often accompanied with a strong decrease of $Q_{0}$ towards the highest gradient reached in the cavity. The main reasons for the performance degradation are excessive heating caused by impurities on the inner surface or by field emission of electrons. The cavity becomes partially normal-conducting, associated with strongly enhanced power dissipation. Because of the exponential increase of surface resistance with temperature this may result in a run-away effect and eventually a quench of the entire cavity.

Field emission of electrons from sharp tips is the most severe limitation in high-gradient superconducting cavities. Small particles on the cavity surface act as field emitters. By applying the clean room techniques developed in semiconductor industry it has been possible to raise the threshold for field emission in multicell cavi- 
ties from about $10 \mathrm{MV} / \mathrm{m}$ to more than $20 \mathrm{MV} / \mathrm{m}$ in the past few years. The preparation of a smooth and almost mirror-like surface by electrolytic polishing is another important improvement.

\subsection{Superconducting materials}

Both magnets and cavities would profit from a superconductor of as high a critical temperature as possible. Technological difficulties have up to now prevented the usage of the ceramic high- $T_{c}$ conductors, hence heliumcooled niobium and its alloys are still the best choice. The most common superconductor used in magnets is niobium-titanium alloy. NbTi magnets are limited to about $6.5 \mathrm{~T}$ at a helium temperature of $4.4 \mathrm{~K}$ (pressurized normal helium) and about $8.5 \mathrm{~T}$ at $2 \mathrm{~K}$ (superfluid helium). In principle niobium-tin $\left(\mathrm{Nb}_{3} \mathrm{Sn}\right)$ would be a better conductor for high-field magnets but the material is very brittle and leads to high costs in the coil production.

The demands on the superconductor in a microwave cavity are rather different. Magnetic flux pinning must be avoided as it is coupled with hysteretic losses in alternating magnetic fields. This excludes an alloy like $\mathrm{NbTi}$, and pure niobium turns out the best sc material. Secondly, a high heat conductivity is needed to guide the heat generated at the inner cavity surface through the wall to the liquid helium coolant. There remain two choices for the cavity layout: the cavity is made from copper and the inner surface is coated with a thin layer of $\mathrm{Nb}$ or, alternatively, the cavity is made from solid $\mathrm{Nb}$. The former approach has been taken with great success with the $350 \mathrm{MHz}$ cavities of the Large Electron Positron ring LEP [13] at CERN. In the TESLA linear collider, however, gradients of more than $25 \mathrm{MV} / \mathrm{m}$ are needed, and these are presently only accessible with cavities made from solid niobium. The material must be of extreme purity with contaminations in the ppm range to achieve a high heat conductivity at $2 \mathrm{~K}$.

\subsection{Outline of the article}

The main goal of this article is to present an introduction into the physical principles and technological challenges of superconducting magnets and cavities for high energy particle accelerators. It is not my intention to give a complete survey of all types of magnets and cavities built in various laboratories and industries. The paper is organized as follows. In Sect. 2 the basics of superconductivity are outlined with special emphasis on the features which are relevant for magnets and microwave cavities. Sect. 3 deals with field calculations of sc accelerator magnets. The layout and performance of practical magnets are described in Sect. 4, the persistent current effects in Sect. 5. The effect of eddy currents in the copper matrix of the superconducting cable and their interplay with the persistent currents are addressed in Sect.
6. Many other interesting and important aspects of superconducting magnets had to be left out. For further reading I refer to the text books Superconducting Magnet Systems by H. Brechna [14], Superconducting Magnets by M.N. Wilson [15], Superconducting Accelerator Magnets by K.-H. Mess, P. Schmüser and S. Wolff [16] and to various review articles [17, 18, 19, 20, 21, 22].

The design principles and basic properties of sc cavities are described in Sect. 7. The observed performance limitations are discussed as well as the measures taken to overcome them. In Sect. 8 the fabrication, preparation and test of practical cavities are presented and the ongoing research and development towards the highest possible accelerating fields is addressed. A topic of considerable interest for practical accelerators is the coupling of rf power into the cavity and the energy transfer to the particle beam. An introduction to these issues is given in Sect. 9. A short appendix deals with thermodynamic properties of superconductors.

For further reading the book RF Superconductivity for Particle Accelerators by H. Padamsee, J. Knobloch and T. Hays [23] and several review articles [24, 25, 26, 27] are recommended.

Within the scope of this article it is impossible to fully cover the enormous amount of work in the field of sc magnets and cavities. I hope my selection was fair. A thorough description of superconductor technology at accelerators can be found in the proceedings of the CERN Accelerator Schools 1988 and 1995 on Superconductivity in Particle Accelerators [28]. Moreover, the Handbook of Accelerator Physics and Technology (A. W. Chao, M. Tigner Ed.) [29] and the proceedings of recent US Particle Accelerator Conferences (PAC) and European Particle Accelerator Conferences (EPAC) contain a wealth of information on new magnet and cavity developments.

\section{Basics of Superconductivity}

The unusual features of superconducting magnets and cavities are closely linked to the physical properties of the superconductor itself. For this reason a basic understanding of superconductivity is indispensable for the design, construction and operation of superconducting accelerator components. Only the traditional 'lowtemperature' superconductors are treated since up to date the use of 'high-temperature' ceramic superconductors in these devices is rather limited [30, 24]. For more comprehensive presentations I refer to the excellent text books by W. Buckel [31] and by D.R. Tilley and J. Tilley [32].

\section{$2.1 \quad$ Overview}

Superconductivity - the infinitely high conductivity below a "critical temperature' $T_{c}$ - is observed in a large variety of materials but, remarkably, not in some of the best normal conductors like copper, silver and gold, except at 
very high pressures. This is illustrated in Fig. 1 where the resistivity of copper, tin and the 'high-temperature" superconductor $\mathrm{YBa}_{2} \mathrm{Cu}_{3} \mathrm{O}_{7}$ is sketched as a function of temperature. Table 2.1 lists some important superconductors together with their critical temperatures at vanishing magnetic field.

\begin{tabular}{|c|c|c|c|c|c|c|c|}
\hline $\mathrm{Al}$ & $\mathrm{Hg}$ & $\mathrm{Sn}$ & $\mathrm{Pb}$ & $\mathrm{Nb}$ & $\mathrm{Ti}$ & $\mathrm{NbTi}$ & $\mathrm{Nb}_{3} \mathrm{Sn}$ \\
1.14 & 4.15 & 3.72 & 7.9 & 9.2 & 0.4 & 9.4 & 18 \\
\hline
\end{tabular}

Table 1: Critical temperature $T_{c}$ in $K$ of selected superconducting materials for vanishing magnetic field.

There is an intimate relation between superconductivity and magnetic fields. W. Meissner and R. Ochsenfeld discovered in 1933 that a superconducting element like lead completely expels a weak magnetic field from its interior when cooled below $T_{c}$, while in stronger fields superconductivity breaks down and the material goes to the normal state. The spontaneous exclusion of magnetic fields upon crossing $T_{c}$ cannot be explained in terms of the Maxwell equations of classical electrodynamics and indeed turned out to be of quantum-theoretical origin. In $1935 \mathrm{H}$. and F. London proposed an equation which offered a phenomenological explanation of the field exclusion. The London equation relates the supercurrent density $J_{s}$ to the magnetic field:

$$
\vec{\nabla} \times \overrightarrow{J_{s}}=-\frac{n_{s} e^{2}}{m_{e}} \vec{B}
$$

where $n_{s}$ is the density of the super-electrons. In combination with the Maxwell equation $\vec{\nabla} \times \vec{B}=\mu_{0} \overrightarrow{J_{s}}$ we get the following equation for the magnetic field in a superconductor

$$
\nabla^{2} \vec{B}-\frac{\mu_{0} n_{s} e^{2}}{m_{e}} \vec{B}=0 .
$$

For a simple geometry, namely the boundary between a superconducting half space and vacuum, and with a magnetic field parallel to the surface, Eq. (2) reads

$$
\frac{d^{2} B_{y}}{d x^{2}}-\frac{1}{\lambda_{L}^{2}} B_{y}=0 \quad \text { with } \quad \lambda_{L}=\sqrt{\frac{m_{e}}{\mu_{0} n_{s} e^{2}}} .
$$

Here we have introduced a very important superconductor parameter, the London penetration depth $\lambda_{L}$. The solution of the differential equation is

$$
B_{y}(x)=B_{0} \exp \left(-x / \lambda_{L}\right)
$$

So the magnetic field does not abruptly drop to zero at the superconductor surface but penetrates into the material with exponential attenuation (Fig. 2). For typical material parameters the penetration depth is quite small, namely $20-50 \mathrm{~nm}$. In the bulk of a thick superconductor the magnetic field vanishes which is just the Meissner-Ochsenfeld effect.
The justification of the London equation remained obscure until the advent of the microscopic theory of superconductivity by Bardeen, Cooper and Schrieffer in 1957. The BCS theory is based on the assumption that the supercurrent is not carried by single electrons but rather by pairs of electrons of opposite momenta and spins, the socalled Cooper pairs. The London penetration depth remains invariant under the replacements $n_{s} \rightarrow n_{c}=n_{s} / 2$, $e \rightarrow 2 e$ and $m_{e} \rightarrow m_{c}=2 m_{e}$.

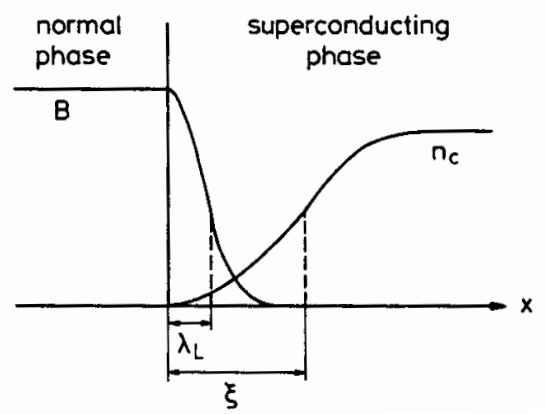

Figure 2: The exponential drop of the magnetic field and the rise of the Cooper-pair density at a boundary between a normal and a superconductor.

The BCS theory revolutionized our understanding of superconductivity. All Cooper pairs occupy a single quantum state, the BCS ground state, whose energy is separated from the single-electron states by an energy gap $2 \Delta(T)$. The critical temperature is related to the energy gap at $T=0$ by

$$
1.76 k_{B} T_{c}=\Delta(0) .
$$

Here $k_{B}=1.38 \cdot 10^{-23} \mathrm{~J} / \mathrm{K}$ is the Boltzmann constant. The magnetic flux through a superconducting ring is found to be quantized, the smallest unit being the elementary flux quantum

$$
\Phi_{0}=\frac{h}{2 e}=2.07 \cdot 10^{-15} \mathrm{Vs} .
$$

These and many other predictions of the BCS theory, like the temperature dependence of the energy gap and the existence of quantum interference phenomena, have been confirmed by experiment and often found practical application.

A discovery of enormous practical consequences was the finding that there exist two types of superconductors with rather different response to magnetic fields. The elements lead, mercury, tin, aluminium and others are called 'type I' superconductors. They do not admit a magnetic field in the bulk material and are in the superconducting state provided the applied field stays below a critical field $H_{c}\left(B_{c}=\mu_{0} H_{c}\right.$ is usually less than 0.1 Tesla). All superconducting alloys like lead-indium, niobium-titanium, niobium-tin and also the element niobium belong to the large class of 'type $\mathrm{II}^{\prime}$ superconductors. They are characterized by two critical fields, $H_{c 1}$ 

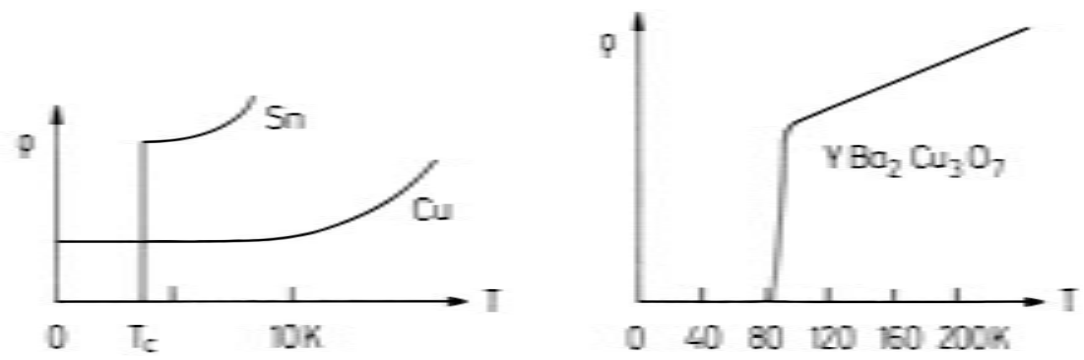

Figure 1: The low-temperature resistivity of copper, tin and $\mathrm{YBa}_{2} \mathrm{Cu}_{3} \mathrm{O}_{7}$.

and $H_{c 2}$. Below $H_{c 1}$ these substances are in the Meissner phase with complete field expulsion while in the range $H_{c 1}<H<H_{c 2}$ they enter the mixed phase in which the magnetic field pierces the bulk material in the form of flux tubes. Many of these materials remain superconductive up to much higher fields (10 Tesla or more).

\subsection{Energy balance in a magnetic field}

A material like lead makes a phase transition from the normal to the superconducting state when it is cooled below $T_{c}$ and when the magnetic field is less than $H_{c}(T)$. This is a phase transition comparable to the transition from water to ice below $0^{\circ} \mathrm{C}$. Phase transitions take place when the new state is energetically favoured. The relevant thermodynamic energy is here the so-called Gibbs free energy $G$ (see appendix A). Free energies have been measured for a variety of materials. For temperatures $T<T_{c}$ they are found to be lower in the superconducting than in the normal state while $G_{\text {sup }}$ approaches $G_{\text {norm }}$ in the limit $T \rightarrow T_{c}$, see Fig. 3a. What is now the impact of a magnetic field on the energy balance? A magnetic field has an energy density $\mu_{0} / 2 \cdot H^{2}$, and according to the Meissner-Ochsenfeld effect the magnetic energy must be pushed out of the material when it enters the superconducting state. Hence the free energy per unit volume in the superconducting state increases quadratically with the applied field:

$$
G_{\text {sup }}(H)=G_{\text {sup }}(0)+\frac{\mu_{0}}{2} H^{2}
$$

while the normal-state energy remains unaffected. The material stays superconductive as long as $G_{\text {sup }}(H)<$ $G_{\text {norm }}$. Equation (7) implies the existence of a maximum tolerable field, the 'critical field', above which superconductivity breaks down. It is defined by the condition that the free energies in the superconducting and in the normal state be equal

$$
G_{\text {sup }}\left(H_{c}\right)=G_{\text {norm }} \Rightarrow \frac{\mu_{0}}{2} H_{c}^{2}=G_{\text {norm }}-G_{\text {sup }}(0) \text {. }
$$

Figure 3 b illustrates what we have said. For $H>H_{c}$ the normal phase has a lower energy, so the material goes to the normal state. Equation (8) is also meaningful for type II superconductors and defines in this case the
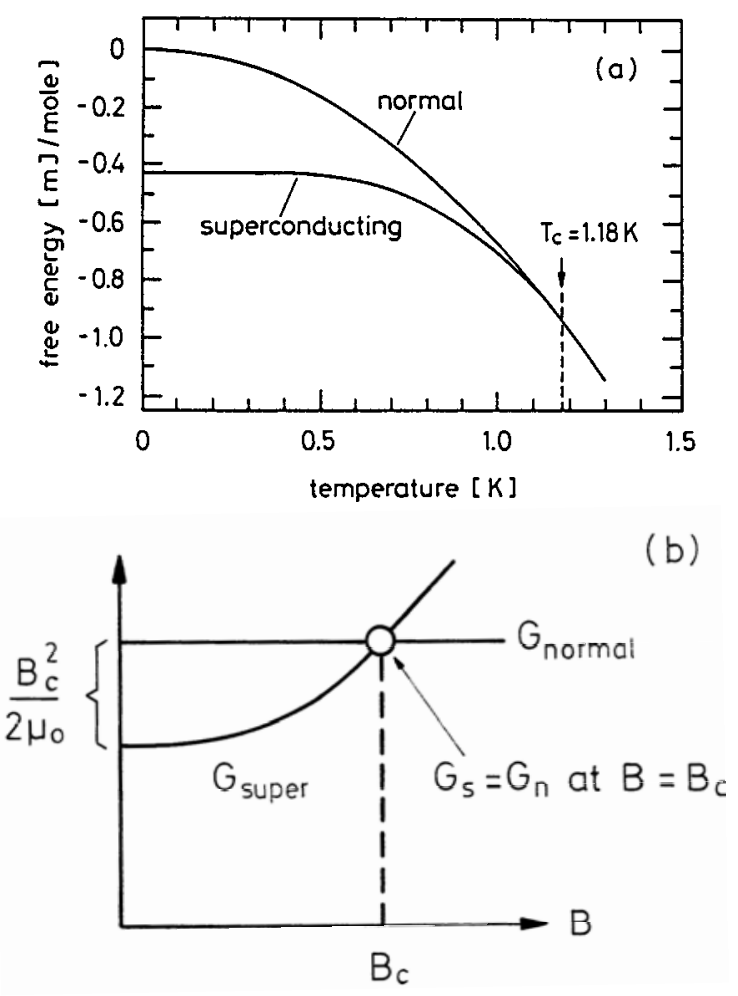

Figure 3: (a) Free energy of aluminium in the normal and superconducting state as a function of $T$ (after N.E. Phillips). The normal state is achieved by applying a magnetic field larger than $B_{c}$. (b) Schematic sketch of the free energies $G_{n o r m}$ and $G_{\text {sup }}$ as a function of the applied magnetic field.

thermodynamic critical field which lies between $H_{c 1}$ and $H_{c 2}$. The quantity $\mu_{0} / 2 \cdot H_{c}^{2}=G_{\text {norm }}-G_{\text {sup }}(0)$ can be interpreted as the Cooper-pair condensation energy per unit volume.

\subsection{Coherence length and distinction be- tween type I and type II supercon- ductors}

In very thin sheets of superconductor (thickness $<\lambda_{L}$ ) the magnetic field does not drop to zero at the centre. Consequently less magnetic energy needs to be expelled which implies that the critical field of a thin sheet may be 
much larger than the $H_{c}$ of a thick slab. From this point of view it might appear energetically favourable for a thick slab to subdivide itself into an alternating sequence of thin normal and superconducting slices. The magnetic energy is indeed lowered that way but there is another energy to be taken into consideration, namely the energy required to create the normal-superconductor interfaces. At the boundary between the normal and the superconducting phase the density $n_{c}$ of the super-current carriers (the Cooper pairs) does not jump abruptly from zero to its value in the bulk but rises smoothly over a finite length $\xi$, called coherence length, see Fig. 2.

The relative size of the London penetration depth $\lambda_{L}$ and the coherence length $\xi$ decides whether a material is a type I or a type II superconductor. Creation of a boundary means a loss of Cooper-pair condensation energy in a thickness $\xi$ but a gain of magnetic energy in a thickness $\lambda_{L}$. There is a net energy gain if $\lambda_{L}>\xi$. So a subdivision of the superconductor into an alternating sequence of thin normal and superconducting slices is energetically favourable if the London penetration depth exceeds the coherence length.

A more refined treatment is provided by the GinzburgLandau theory (see e.g. [32]). Here one introduces the Ginzburg-Landau parameter

$$
\kappa=\lambda_{L} / \xi
$$

The criterion for type I or II superconductivity is found to be

$$
\begin{aligned}
\text { type I: } & \kappa<1 / \sqrt{2} \\
\text { type II: } & \kappa>1 / \sqrt{2} .
\end{aligned}
$$

The following table lists the penetration depths and coherence lengths of some important superconducting elements. Niobium is a type II conductor but close to the border to type I, while indium, lead and tin are clearly in the type I class.

\begin{tabular}{|c|cccc|}
\hline material & In & $\mathrm{Pb}$ & $\mathrm{Sn}$ & $\mathrm{Nb}$ \\
\hline$\lambda_{L}[\mathrm{~nm}]$ & 24 & 32 & $\approx 30$ & 32 \\
\hline$\xi[\mathrm{nm}]$ & 360 & 510 & $\approx 170$ & 39 \\
\hline
\end{tabular}

The coherence length $\xi$ is proportional to the mean free path of the conduction electrons in the metal. In alloys the mean free path is generally much shorter than in pure metals hence alloys are always type II conductors.

In reality a type II superconductor is not subdivided into thin slices but the field penetrates the sample in the form of flux tubes which arrange themselves in a triangular pattern which can be made visible by evaporating iron atoms onto a superconductor surface sticking out of the liquid helium. The fluxoid pattern shown in Fig. 4a proves beyond any doubt that niobium is indeed a type II superconductor. Each flux tube or fluxoid contains one elementary flux quantum $\Phi_{0}$ which is surrounded by a Cooper-pair vortex current. The centre of a fluxoid is normal-conducting and covers an area of roughly $\pi \xi^{2}$. When we apply an external field $H$ fluxoids keep moving into the specimen until their average magnetic flux density is identical to $B=\mu_{0} H$. The fluxoid spacing in the triangular lattice $d=\sqrt{2 \Phi_{0} /(\sqrt{3} B)}$ amounts to $20 \mathrm{~nm}$ at 6 Tesla. The upper critical field is reached when the current vortices of the fluxoids start touching each other at which point superconductivity breaks down. In the Ginzburg-Landau theory the upper critical field is given by

$$
B_{c 2}=\sqrt{2} \kappa B_{c}=\frac{\Phi_{0}}{2 \pi \xi^{2}}
$$

For niobium-titanium with an upper critical field $B_{c 2}=$ $14 \mathrm{~T}$ this formula yields $\xi=5 \mathrm{~nm}$. The coherence length is larger than the typical width of a grain boundary in NbTi which means that the supercurrent can freely move from grain to grain. In high- $T_{c}$ superconductors the coherence length is often shorter than the grain boundary width, and then current flow from one grain to the next is strongly impeded.

\subsection{Flux flow resistance and flux pinning}

For application in accelerator magnets a superconducting wire must be able to carry a large current in the presence of a field of $5-10$ Tesla. Type I superconductors are definitely ruled out because their critical field is far too low (below 0.1 Tesla). Type II conductors appear promising at first sight: they feature large upper critical fields, and high currents are permitted to flow in the bulk material. However there is the problem of flux flow resistance. A current flowing through an ideal type II superconductor, which is exposed to a magnetic field, exerts a Lorentz force on the flux lines and causes them to move through the specimen, see Fig. 4b. This is a viscous motion and leads to heat generation. So although the current itself flows without dissipation the sample acts as if it had an Ohmic resistance. The statement is even formally correct. The moving fluxoids represent a moving magnetic field which, according to theory of special relativity, is equivalent to an electric field $\vec{E}_{\text {equiv }}=\vec{B} \times \vec{v} / c^{2}$. It is easy to see that $\vec{E}_{\text {equiv }}$ and $\vec{J}$ point in the same direction just like in a normal resistor. Flux flow resistance has been experimentally established [33].

To obtain useful wires for magnet coils flux flow has to be prevented by capturing the fluxoids at pinning centres. These are defects or impurities in the regular crystal lattice. The most important pinning centres in niobiumtitanium are normal-conducting titanium precipitates in the so-called $\alpha$ phase whose size is in the range of the fluxoid spacing ( $\approx 10 \mathrm{~nm}$ at 6 Tesla). Figure 5 shows a microscopic picture of a conductor with very high current density $\left(3700 \mathrm{~A} / \mathrm{mm}^{2}\right.$ at $5 \mathrm{~T}$ and $\left.4.2 \mathrm{~K}\right)$.

A type II superconductor with strong pinning is called a hard superconductor. Hard superconductors are very well suited for high-field magnets, they permit dissipationless current flow in high magnetic fields. There is a penalty, however: these conductors exhibit a strong magnetic hysteresis which is the origin of the very annoying 

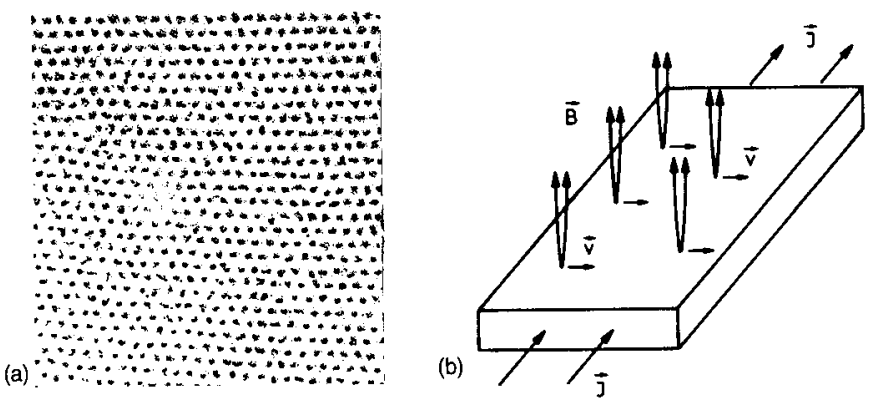

Figure 4: (a) Fluxoid pattern in niobium (courtesy U. EsSmann). The distance between adjacent flux tubes is $0.2 \mu \mathrm{m}$. (b) Fluxoid motion in a current-carrying type II superconductor.

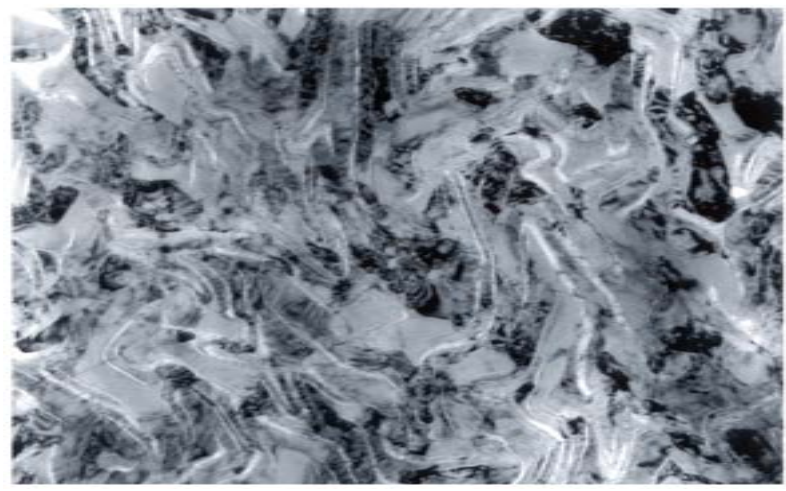

Figure 5: Micrograph of NbTi. The $\alpha$-titanium precipitates appear as lighter strips. The area covered is $840 \mathrm{~nm}$ wide and $525 \mathrm{~nm}$ high. Courtesy P.J. Lee and D.C. Larbalestier.

'persistent-current' multipoles in superconducting accelerator magnets.

\subsection{Magnetization of a hard supercon- ductor}

A type I superconductor shows a reversible response ${ }^{3}$ to a varying external magnetic field $H$. The magnetization is given by the straight line $M(H)=-H$ for $0<H<H_{c}$ and then drops to zero. An ideal type II conductor without any flux pinning should also react reversibly. A hard superconductor, on the other hand, is only reversible in the Meissner phase because then no magnetic field enters the bulk, so no flux pinning can happen. If the field is raised beyond $H_{c 1}$ magnetic flux enters the sample and is captured at pinning centres. When the field is reduced again these flux lines remain bound and the specimen keeps a frozen-in magnetization even for vanishing external field. One has to invert the field polarity to achieve $M=0$ but the initial state $(H=0$ and no captured flux in the bulk material) can only be recovered by warming up the specimen to destroy superconductiv-

\footnotetext{
${ }^{3}$ This statement applies only for long cylindral or elliptical samples oriented parallel to the field.
}

ity and release all pinned flux quanta, and by cooling down again.

A typical hysteresis curve is shown in Fig. 6. There is a close resemblence with the hysteresis in iron except for the sign: the magnetization in a superconductor is opposed to the magnetizing field because the physical mechanism is diamagnetism. The magnetic hysteresis is

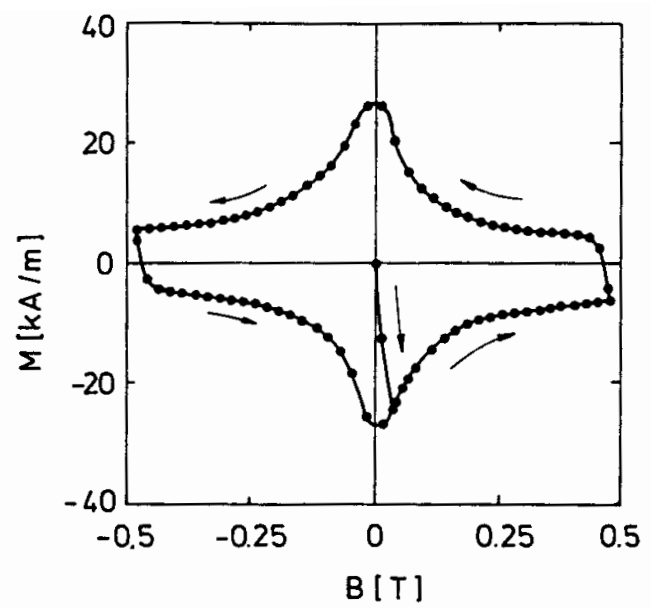

Figure 6: Measured magnetization $M$ of a multifilamentary niobium-titanium conductor [34]. Shown is the initial excitation, starting at $B=\mu_{0} H=0$ and $M=0$, and the magnetic hysteresis for an external field $B$ varying between $+0.5 \mathrm{~T}$ and $-0.5 \mathrm{~T}$. Note that the hysteresis curve is not exactly symmetric with respect to the horizontal axis. The slight asymmetry is due to surface currents (Meissner-Ochsenfeld effect) whose magnetic moment is always opposed to the applied field.

associated with energy dissipation. When a hard superconductor is exposed to a time-varying field and undergoes a cycle like the loop in Fig. 6, the energy loss is given by the integral

$$
Q_{\text {hyst }}=\oint \mu_{0} M(H) d H .
$$

It is equal to the area enclosed by the loop. This energy must be provided by the power supply of the fieldgenerating magnet and is transformed into heat in the superconductor when magnetic flux quanta are moved in and out of the specimen.

\subsection{Critical current density}

For a hard superconductor, not only temperature $T$ and magnetic field $H$ have to be specified but also current density $J$. The material can be conveniently characterized by its critical surface in a $(T, H, J)$ coordinate system. For the most important conductor used in magnets, niobium-titanium, this surface is depicted in Fig. 7. Superconductivity prevails everywhere below the surface and normal conductivity above it. A hard superconductor is not exactly free of any resistance. The critical current density (at a given temperature and field) 
is usually defined by the criterion that the resistivity be $\rho=\rho_{c}=10^{-14} \Omega \mathrm{m}$. In the vicinity of this point the resistivity is a very steep function of current density. It can be parametrised with a power law

$$
\rho(J)=\rho_{c}\left(\frac{J}{J_{c}}\right)^{n} .
$$

The exponent $n$ is a quality index which may be as large as 50 for a good multifilamentary NbTi conductor.

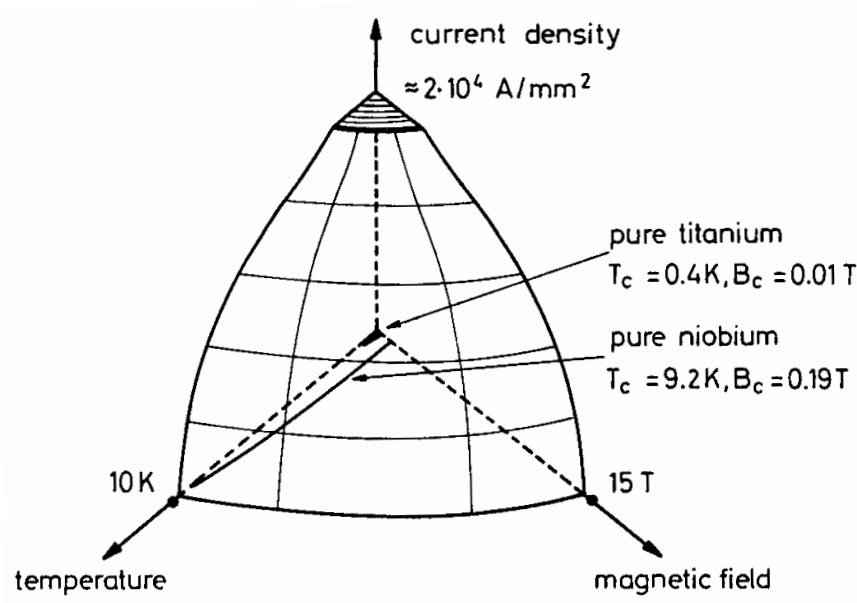

Figure 7: Sketch of the critical surface of NbTi. Also indicated are the regions where pure niobium and pure titanium are superconducting. The critical surface has been truncated in the regime of very low temperatures and fields where only sparse data are available.

\subsection{Superconductors in microwave fields}

Superconductivity in microwave fields is not treated adequately in standard text books. For this reason I present in this section a simplified explanation of the important concepts. A similar treatment can be found in [23]. Superconductors are free from energy dissipation in directcurrent (dc) applications, but this is no longer true for alternating currents (ac) and particularly not in microwave fields. The reason is that the high-frequency magnetic field penetrates a thin surface layer and induces oscillations of the electrons which are not bound in Cooper pairs. The power dissipation caused by the motion of the unpaired electrons can be characterized by a surface resistance. In copper cavities the surface resistance is given by (see sect. 7.4 .3 )

$$
R_{\text {surf }}=\frac{1}{\delta \sigma}
$$

where $\delta$ is the skin depth and $\sigma$ the conductivity of the metal.

The response of a superconductor to an ac field can be understood in the framework of the two-fluid model ${ }^{4}$.

\footnotetext{
${ }^{4} \mathrm{~A}$ similar model is used to explain the unusual properties of
}

An ac current in a superconductor is carried by Cooper pairs (the superfluid component) as well as by unpaired electrons (the normal component). Let us study the response to a periodic electric field. The normal current obeys Ohm's law

$$
J_{n}=\sigma_{n} E_{0} \exp (i \omega t)
$$

while the Cooper pairs receive an acceleration $m_{c} \dot{v}_{c}=$ $-2 e E_{0} \exp (i \omega t)$, so the supercurrent density becomes

$$
J_{s}=-i \frac{n_{c} 2 e^{2}}{m_{e} \omega} E_{0} \exp (i \omega t) .
$$

If we write for the total current density

$$
J=J_{n}+J_{s}=\sigma E_{0} \exp (i \omega t)
$$

we get a complex conductivity:

$$
\sigma=\sigma_{n}-i \sigma_{s} \quad \text { with } \quad \sigma_{s}=\frac{2 n_{c} e^{2}}{m_{e} \omega}=\frac{1}{\mu_{0} \lambda_{L}^{2} \omega} .
$$

We know already that the rf magnetic field penetrates a superconductor much less than a normal conductor, namely only to a depth $\lambda_{L}$. The surface resistance is the real part of the complex surface impedance

$$
R_{\text {surf }}=\operatorname{Re}\left(\frac{1}{\lambda_{L}\left(\sigma_{n}-i \sigma_{s}\right)}\right)=\frac{1}{\lambda_{L}} \cdot \frac{\sigma_{n}}{\sigma_{n}^{2}+\sigma_{s}^{2}} .
$$

Since $\sigma_{n}^{2} \ll \sigma_{s}^{2}$ at microwave frequencies one can disregard $\sigma_{n}^{2}$ in the denominator and obtains $R_{\text {surf }} \propto$ $\sigma_{n} /\left(\lambda_{L} \sigma_{s}^{2}\right)$. So we arrive at the surprising result that the microwave surface resistance is proportional to the normal-state conductivity.

The conductivity of a normal metal is given by the classic Drude expression

$$
\sigma_{n}=\frac{n_{n} e^{2} \ell}{m_{e} v_{F}}
$$

where $n_{n}$ is the density of the unpaired electrons, $\ell$ their mean free path and $v_{F}$ the Fermi velocity. The normal electrons are created by thermal breakup of Cooper pairs. There is an energy gap $E_{g}=2 \Delta(T)$ between the BCS ground state and the free electron states. By analogy with the conductivity of an intrinsic (undoped) semiconductor we get $n_{n} \propto \exp \left(-E_{g} /\left(2 k_{B} T\right)\right)$ and hence

$$
\sigma_{n} \propto \ell \exp \left(-\Delta(T) /\left(k_{B} T\right)\right) .
$$

Using $1 / \sigma_{s}=\mu_{0} \lambda_{L}^{2} \omega$ and $\Delta(T) \approx \Delta(0)=1.76 k_{B} T_{c}$ we finally obtain for the BCS surface resistance

$$
R_{B C S} \propto \lambda_{L}^{3} \omega^{2} \ell \exp \left(-1.76 T_{c} / T\right) .
$$

This formula displays two important aspects of microwave superconductivity: the surface resistance depends exponentially on temperature, and it is proportional to the square of the rf frequency.

liquid helium below $2.17 \mathrm{~K}$ in terms of a normal and a superfluid component. 


\section{Field Calculations for Acceler- ator Magnets}

The field calculation for superconducting magnets is quite different from that of normal magnets with iron yokes. The mathematical methods are elegant and of general interest, so I present them in some detail.

\subsection{Multipole expansion for a single cur- rent conductor}

A schematic view of a superconducting dipole for a large accelerator is given in Fig. 8. The length of the magnet

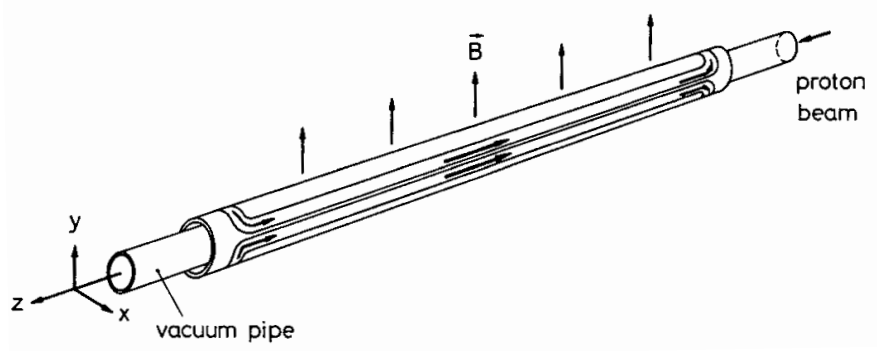

Figure 8: Schematic view of a superconducting dipole coil.

is much larger than its aperture and the current conductors run parallel to the beam over the longest part of the magnet, except for the short coil heads. The dipole magnets follow the beam orbit but the deviation from a straight line is small so one can consider the magnetic field essentially as two-dimensional and apply the theory of analytic functions. In a region in space which is free of any currents and magnetized materials, the magnetic field fulfils the following two equations

$$
\vec{\nabla} \cdot \vec{B}=0, \quad \vec{\nabla} \times \vec{B}=0 .
$$

This implies that we can express $\vec{B}$ either as the curl of the familiar vector potential $\vec{A}$ or as the gradient of a scalar magnetic potential $V$

$$
\vec{B}=\vec{\nabla} \times \vec{A}, \quad \vec{B}=-\vec{\nabla} V .
$$

For our two-dimensional problem the vector potential has only a $z$ component. The $x$ and $y$ components of the magnetic field vector can be computed in two ways

$$
B_{x}=-\frac{\partial V}{\partial x}=\frac{\partial A_{z}}{\partial y}, \quad B_{y}=-\frac{\partial V}{\partial y}=-\frac{\partial A_{z}}{\partial x} .
$$

The complex potential function

$$
\tilde{A}(x, y)=A_{z}(x, y)+i V(x, y)
$$

is an analytic function of the complex variable $\zeta=x+i y$ since the equations (24) are identical with the CauchyRiemann conditions for the real and imaginary part of an analytic function. So one can expand $\tilde{A}$ in a power series about the origin

$$
\tilde{A}(x, y)=\sum_{n=0}^{\infty} c_{n}(x+i y)^{n}
$$

which converges in the largest circle which contains neither current nor magnetized material. Taking the derivatives of (26), we obtain the multipole expansions for the components of the magnetic field vector ${ }^{5}$.

Having shown that any two-dimensional field in vacuum can be expanded in a multipole series, we now compute this series explicitely for a very simple case, namely a single current-carrying wire. The magnet axis is chosen as the $z$ direction of a cylindrical coordinate system $(r, \theta, z)$, shown in Fig. 9a. In the almost straight section of the magnet all current conductors are parallel to the $z$ axis and can be considered as infinitely long since the transverse dimensions are small. Consider first a line current in the positive $z$ direction which flows exactly on the $z$ axis. The magnetic field lines are concentric circles around the $z$ axis, so the field is purely azimuthal and has the familiar form $B_{\theta}=\mu_{0} I /(2 \pi r)$. The vector potential generated by the current has only a $z$ component: $A_{z}(r, \theta)=-\mu_{0} I \ln r /(2 \pi)$.

Take now a current parallel to the $z$ axis but located at an arbitrary point $(r=a, \theta=\phi)$ in the $(r, \theta)$ plane, see Fig. 9b. The vector potential has almost the same form:

$$
A_{z}(r, \theta)=-\frac{\mu_{0} I}{2 \pi} \ln \left(\frac{R}{a}\right)
$$

with $R=\sqrt{a^{2}+r^{2}-2 a r \cos (\theta-\phi)}$ the distance between the current and the point $P=(r, \theta)$ at which we want to evaluate $A_{z}$. Let us consider the case $r<a$.
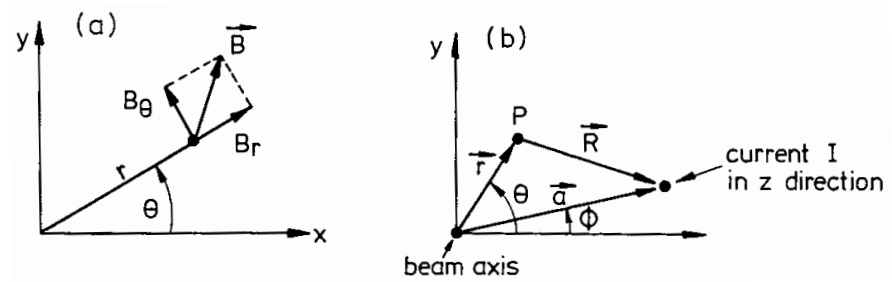

Figure 9: (a) Coordinate system for the multipole expansion. (b) Field calculation for a line current.

Then we write with $\alpha=\theta-\phi$

$$
R^{2}=a^{2}[1-(r / a) \exp (i \alpha)][1-(r / a) \exp (-i \alpha)]
$$

\footnotetext{
${ }^{5}$ Another frequently used possibility is to define an analytic function by $\tilde{B}=B_{y}+i B_{x}$. We prefer the vector potential since a single scalar quantity, $A_{z}$ in this case, is sufficient to compute the magnetic field pattern. For current-dominated magnets the vector potential is in fact very practical [14] because the vector $\vec{A}$ is parallel to the current density $\vec{J}$. For conventional magnets the scalar potential is more adequate because the iron pole shoes are equipotential surfaces for $\mu \gg 1$.
} 
$\ln (R / a)=\frac{1}{2} \ln [1-(r / a) \exp (i \alpha)]+\frac{1}{2} \ln [1-(r / a) \exp (-i \alpha)]$

Now we use the Taylor expansion of the logarithm

$$
\ln (1-\xi)=-\xi-\frac{1}{2} \xi^{2}-\frac{1}{3} \xi^{3}-\ldots-\frac{1}{n} \xi^{n}-\ldots
$$

which converges for arbitrary complex numbers $\xi$ with $|\xi|<1$. The vector potential for $r<a$ is then given by the multipole expansion

$$
A_{z}(r, \theta)=\frac{\mu_{0} I}{2 \pi} \sum_{n=1}^{\infty} \frac{1}{n}\left(\frac{r}{a}\right)^{n} \cos [n(\theta-\phi)] .
$$

A single line current produces multipole fields of all orders $n$.

\subsection{Generation of pure multipole fields}

We consider now an arrangement of current conductors, running parallel to the $z$ direction, which are mounted on a cylinder of radius $a$. A pure multipole field, containing just the single order $n=m$, is obtained inside the cylinder if the current distribution as a function of the azimuthal angle $\phi$ is given by

$$
I(\phi)=I_{0} \cos (m \phi) \text {. }
$$

The statement is easily proved by computing the vector potential resulting from the current distribution (29):

$A_{z}(r, \theta)=\frac{\mu_{0} I_{0}}{2 \pi} \sum_{n=1}^{\infty} \frac{1}{n}\left(\frac{r}{a}\right)^{n} \int_{0}^{2 \pi} \cos (m \phi) \cos [n(\theta-\phi)] d \phi$.

From $\cos [n(\theta-\phi)]=\cos (n \theta) \cos (n \phi)+\sin (n \theta) \sin (n \phi)$ and the orthogonality of the trigonometric functions follows immediately that the integral vanishes unless $n=$ $m$, so only a single term in the sum remains:

$$
A_{z}(r, \theta)=\frac{\mu_{0} I_{0}}{2} \cdot \frac{1}{m}\left(\frac{r}{a}\right)^{m} \cos (m \theta) .
$$

The magnetic field components are

$$
\begin{aligned}
& B_{\theta}(r, \theta)=-\frac{\partial A_{z}}{\partial r}=-\frac{\mu_{0} I_{0}}{2 a}\left(\frac{r}{a}\right)^{m-1} \cos (m \theta) \\
& B_{r}(r, \theta)=\frac{1}{r} \frac{\partial A_{z}}{\partial \theta}=-\frac{\mu_{0} I_{0}}{2 a}\left(\frac{r}{a}\right)^{m-1} \sin (m \theta) .
\end{aligned}
$$

For $m=1$ and 2 we obtain dipole and quadrupole fields. The coils are shown in Fig. 10, together with the iron yokes of the corresponding normal magnets. The fields (31) are called normal multipole fields. If we rotate the current distribution (29) by an angle of $\pi /(2 m)$, we obtain a $\sin (m \phi)$ distribution leading to skew multipole fields. A skew dipole, for instance, has a horizontal field. Such magnets are needed to correct the particle orbit in the vertical plane. All other skew multipoles are quite undesirable in an accelerator. Skew quadrupole fields arise from an angular misalignment of the normal quadrupoles. They have the unpleasant feature of coupling horizontal and vertical betatron oscillations. A few correction quadrupoles, rotated by $45^{\circ}$ around their axis, are usually needed to eliminate the coupling.

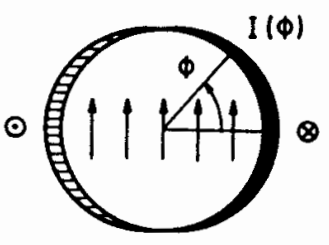

Quadrupole $1(\Phi)=I_{0} \cos 2 \Phi$

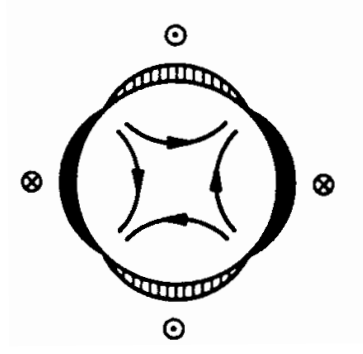

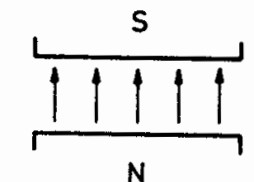

N

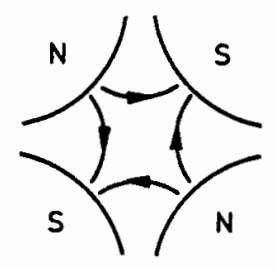

Figure 10: Generation of pure dipole and quadrupole fields by $\cos (\phi)$ resp. $\cos (2 \phi)$ current distributions and by conventional magnets with iron pole shoes.

\subsection{Approximation of pure multipole coils by current shells}

Current distributions with a $\cos (m \phi)$ dependence are difficult to fabricate with a superconducting cable of constant cross section. They can be approximated with sufficient accuracy by current shells or current blocks.

The quality of such an approximation can be judged from the general multipole expansion:

$$
\begin{aligned}
& B_{\theta}(r, \theta)=B_{\text {ref }} \sum_{n=1}^{\infty}\left(\frac{r}{r_{0}}\right)^{n-1}\left[b_{n} \cos (n \theta)-a_{n} \sin (n \theta)\right], \\
& B_{r}(r, \theta)=B_{\text {ref }} \sum_{n=1}^{\infty}\left(\frac{r}{r_{0}}\right)^{n-1}\left[a_{n} \cos (n \theta)+b_{n} \sin (n \theta)\right] .
\end{aligned}
$$

Here $r_{0}$ is a reference radius, which should be in the same order as the maximum deviation of the particles from the centre axis of the magnet. The quantity $B_{\text {ref }}$ is a reference field, namely the magnitude of the main field at the reference radius, so $B_{r e f}=B_{1}$ for a dipole and $B_{r e f}=B_{2}\left(r_{0}\right)=g \cdot r_{0}$ for a quadrupole $(g$ is the gradient). The $b_{n}$ are called the normal multipole coefficients, the $a_{n}$ are the skew coefficients ${ }^{6}$. With the above choice of $B_{\text {ref }}$ the main coefficient is normalized to unity: $b_{1}=1$ in a dipole, $b_{2}=1$ in a quadrupole. The remaining coefficients should be very small for a good magnet, typically $\left|a_{n}\right|,\left|b_{n}\right|<1 \cdot 10^{-4}$.

\footnotetext{
${ }^{6}$ In the American literature it is convention to start the multipole series with $n=0: B_{y}+i B_{x}=B_{\text {ref }} \sum_{n=0}^{\infty}\left(b_{n}+\right.$ $\left.i a_{n}\right)(x+i y)^{n} / r_{0}^{n}$. The multipole indices are therefore lower by one unit, so a normal dipole, quadrupole, sextupole is denoted by $b_{0}, b_{1}, b_{2}$, respectively, and similarly for the skew multipoles.
} 
We observe that the ideal multipole coils of Fig. 10 have well defined symmetries. In a dipole coil, for any line current $+I$ at an angle $\phi$, there exist three more currents: $+I$ at $-\phi$ and $-I$ at $\pi-\phi$ and $\pi+\phi$, see Fig. 11a. The vector potential of these four currents, using Eq. (28), is

$$
A_{z}(r, \theta)=\frac{2 \mu_{0} I}{\pi} \sum_{n=1,3,5, \ldots} \frac{1}{n}\left(\frac{r}{a}\right)^{n} \cos (n \phi) \cos (n \theta) .
$$

Only the $\cos (n \theta)$ terms remain, hence we can draw an important conclusion: a coil with dipole symmetry possesses only normal multipoles of odd order. Even orders and skew multipoles are absent. Similarly, a coil with quadrupole symmetry has only normal multipoles and the 'allowed' orders are odd multiples of the lowest order 2: $n=2,6,10,14, \ldots$. (a)

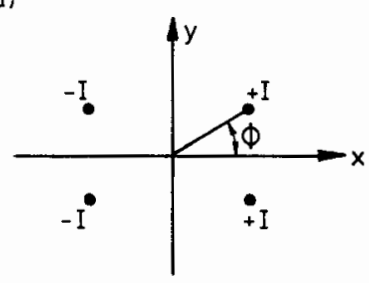

(b)

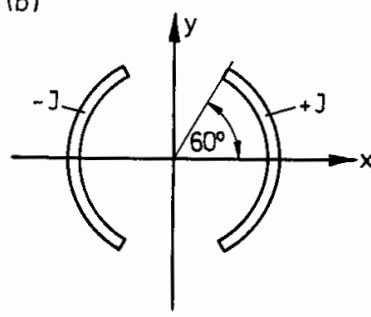

Figure 11: (a) Four line currents with dipole symmetry. (b) Simplest current-shell arrangement for a dipole coil.

The simplest current shell arrangement with dipole symmetry is shown in Fig. 11b. We take a constant current density $J$ and compute the vector potential inside the coil using Eq. (33)

$A_{z}(r, \theta)=C \sum_{n} \frac{\cos (n \theta)}{n} \int_{a_{1}}^{a_{2}}\left(\frac{r}{a}\right)^{n} a d a \int_{0}^{\phi_{l}} \cos (n \phi) d \phi$

with $C=2 \mu_{0} J / \pi$. The limiting angle of the current shell is denoted by $\phi_{l}$ and the radii are $a_{1}, a_{2}$. The integrations can be done analytically. For a thin current shell with $\Delta a=a_{2}-a_{1} \ll a=\frac{1}{2}\left(a_{1}+a_{2}\right)$ the result is

$A_{z}(r, \theta)=\frac{2 \mu_{0} J a \Delta a}{\pi} \sum_{n=1,3,5, \ldots} \frac{1}{n^{2}}\left(\frac{r}{a}\right)^{n} \sin \left(n \phi_{l}\right) \cos (n \theta)$.

The multipole coefficients of this coil are

$$
b_{n}=\frac{1}{n}\left(\frac{r_{0}}{a}\right)^{n-1} \frac{\sin \left(n \phi_{l}\right)}{\sin \left(\phi_{l}\right)}, \quad n=1,3,5, \ldots
$$

Choosing a limiting angle of $\phi_{l}=60^{\circ}$ the sextupole term $b_{3}$ vanishes. Then the first non-vanishing higher multipole is the decapole $n=5$. For typical coil dimensions $b_{5}$ is a few percent, two orders of magnitude larger than is tolerable. A single-layer current shell with constant current density is therefore too rough an approximation for a dipole coil. With two current shells, the sextupole and decapole can both be made to vanish by choosing a limiting angle of about $72^{\circ}$ in the inner and of $36^{\circ}$ in the outer layer. The Tevatron dipoles are built this way. There remain higher coefficients $\left(b_{7}, b_{9}\right)$ which are in the order of $10^{-3}$. A further reduction of these and all higher multipoles below the $10^{-4}$ level is possible by placing wedge-shaped insulators into the inner and outer coil layer. The HERA and LHC magnets are constructed in this manner (Fig. 12a).

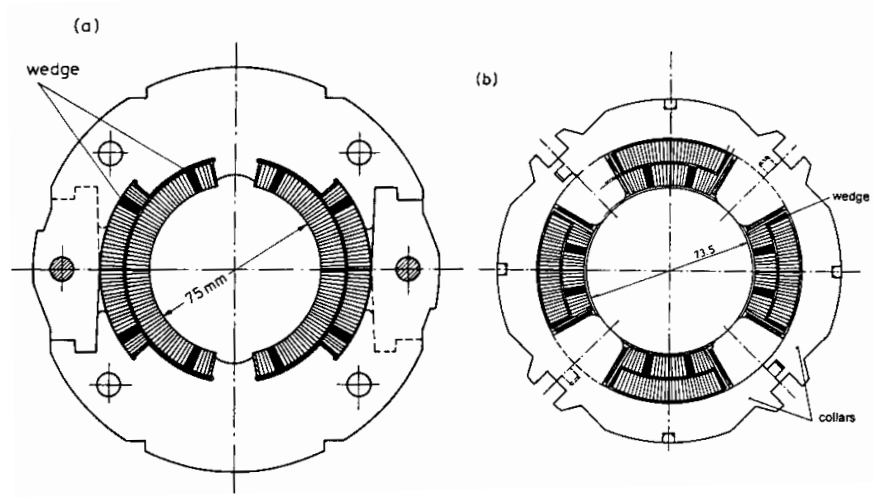

Figure 12: (a) A two-shell dipole coil with longitudinal wedges in the inner and outer layer for improved field homogeneity. The coil is confined by non-magnetic collars. Coils of this type are used in the HERA and LHC magnets.

(b) Cross section of a quadrupole coil (Tevatron, HERA).

A single-layer quadrupole coil has a vanishing 12-pole $(n=6)$ but a $b_{10}$ of about $2 \%$. In the Tevatron and HERA quadrupoles two shells with additional wedges are used (Fig. 12b), and then most of the higher multipoles are in the $10^{-4}$ range or lower.

\subsection{Iron yoke}

The dipole and quadrupole magnets are equipped with an iron yoke with a cylindrical inner bore which confines the magnetic field. Its influence on the field at the particle beam can be analyzed with the method of image currents provided the iron is not saturated and the permeability $\mu$ is uniform. Consider a current $I$ at a radius $a$ inside a hollow iron yoke whose inner surface is a cylinder of radius $R_{y}$. The effect of the iron on the inner field is equivalent to that of an image current $I^{\prime}$ which is located at the image radius $a^{\prime}=R_{y}^{2} / a$ :

$$
I^{\prime}=\frac{\mu-1}{\mu+1} \cdot I, \quad a^{\prime}=\frac{R_{y}^{2}}{a} .
$$

The image current $I^{\prime}$ flows parallel to the real current $I$ and enhances the inner field. Fig. 13 shows the images of a single line current and of a current shell. In the latter case the image current density is lower due to the increased area. 


$$
J^{\prime}=\frac{\mu-1}{\mu+1} \cdot J \cdot\left(\frac{a}{R_{y}}\right)^{4} \quad \text { with } \quad a=\sqrt{a_{1} a_{2}}
$$
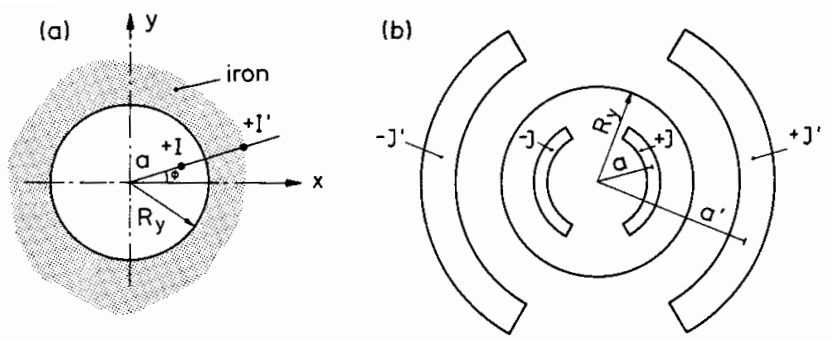

Figure 13: (a) Image of a line current inside a hollow iron yoke. (b) Image of a single-shell dipole coil.

For a single-layer dipole coil with concentric iron yoke the dipole component is

$$
B_{1}=\frac{2 \mu_{0} \sin \left(\phi_{l}\right)}{\pi}\left(J \Delta a+J^{\prime} \Delta a^{\prime}\right) .
$$

Here the first term in the bracket is the coil contribution and the second term the iron contribution. Now $J^{\prime} \Delta a^{\prime}=$ $(\mu-1) /(\mu+1) \cdot J \Delta a \cdot\left(a / R_{y}\right)^{2}$. So for $\mu \gg 1$

$$
\left(B_{1}\right)_{\text {iron }} /\left(B_{1}\right)_{\text {coil }}=\left(a / R_{y}\right)^{2} .
$$

As a simple example we consider the inner coil shell in the HERA dipole with an average radius $a=42.5 \mathrm{~mm}$ and a yoke radius $R_{y}=88.4 \mathrm{~mm}$. In this case the relative iron contribution to the total dipole field on the axis is $19 \%$.

For higher multipole orders $n$ the iron contribution is much smaller:

$$
\left(B_{n}\right)_{i r o n} /\left(B_{n}\right)_{c o i l}=\left(a^{2} / R_{y}^{2}\right)^{n} .
$$

For the sextupole field $B_{3}$ this amounts to about $1.3 \%$ in the above example. The normalized sextupole coefficient $b_{3}=B_{3} / B_{1}$, however, is reduced by about $18 \%$ because of the $19 \%$ iron contribution to the dipole field. Note that in a two-layer coil the sextupole and the allowed higher poles are modified by the yoke because the mirror image inverts the inner and outer coils. The limiting angles of the coil shells are adjusted in such a way that the sextupole vanishes when the coil is mounted inside the yoke. An important observation is that an unsaturated iron yoke does not create any new multipoles.

\subsection{Iron yoke saturation}

The image current method fails when the iron yoke approaches saturation and the permeability $\mu$ becomes position-dependent. Finite element programs are needed to compute the field pattern. With iron saturation the relation between dipole field $B_{1}$ and current $I$ is no longer linear and current-dependent sextupole and decapole coefficients arise. The saturation effects depend strongly on the proximity between coil and yoke. Three typical cases shall be considered.

\subsection{1 'Warm-iron' dipole}

In the Tevatron magnets (Fig. 14) the yoke is outside the cryostat and thus fairly far away from the coil. In this type of magnet saturation is almost negligible up to the critical current of the conductor. The iron contribution to the dipole field is about $10 \%$; the field depends linearly on the current and no higher multipoles are observed.

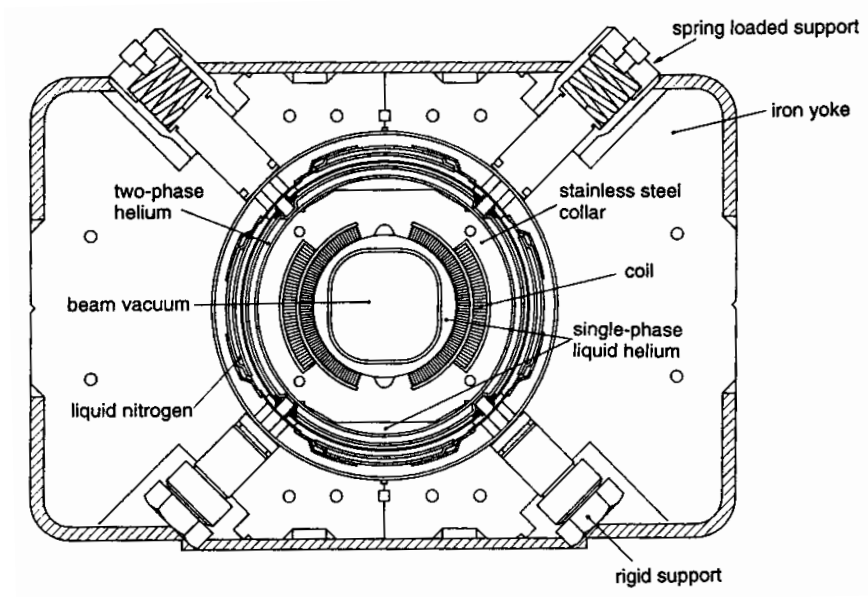

Figure 14: The Tevatron 'warm-iron' dipole [17].

\subsection{2 'Cold-iron' dipole}

For the RHIC collider a dipole was developed type whose coil is surrounded by a soft-iron yoke that is contained in the liquid helium cryostat. The yoke contributes about $35 \%$ to the central field, so a substantial saving in superconductor is possible. In the first version of the RHIC dipole strongly current-dependent sextupole and decapole coefficients were present but in the final design considerable progress has been achieved by increasing the thickness of the glass-phenolic spacer between coil and yoke and by punching holes into the iron yoke laminations at suitable positions. In the range of operation the saturation-induced multipoles deviate from the average by only $\pm 2.5 \cdot 10^{-4}$ for $b_{3}$ and $\pm 0.4 \cdot 10^{-4}$ for $b_{5}$, see Fig. 15.

\subsection{3 'HERA-type' dipole}

A third type, devised at DESY, combines the coil of the warm-iron design, confined by non-magnetic clamps, with an iron yoke inside the cryostat (Fig. 16). Here, the non-linearity in field versus current is quite moderate $(<0.5 \%$ at $6 \mathrm{~T})$ and the sextupole variation stays below $1 \cdot 10^{-4}$. 
In the Large Hadron Collider LHC the two counterrotating proton beams are bent and focused by twinaperture magnets, having two coils of opposite polarity in a common iron yoke. A cross section is shown in Fig. 17. At the design field of $8.36 \mathrm{~T}$, corresponding to a proton energy of $7000 \mathrm{GeV}$, there is significant iron saturation in the centre region. The resulting normal quadrupole component is minimized by a suitable hole pattern in the iron yoke. The remaining $b_{2}$ of about $2 \cdot 10^{-4}$ at high field is compensated by the quadrupole magnets in the LHC ring which are powered independently from the dipoles.
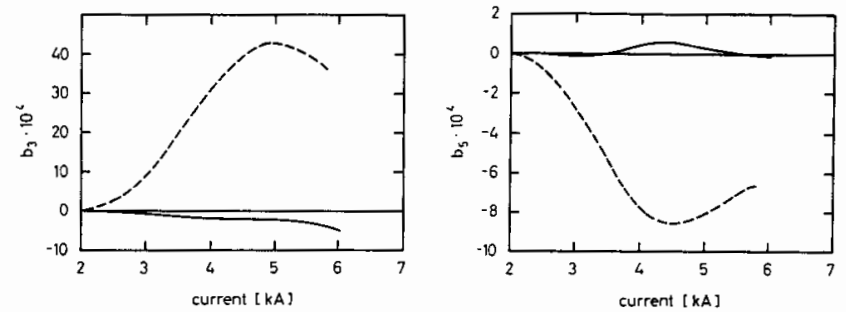

Figure 15: The current dependence of $b_{3}$ and $b_{5}$ in the first design (dotted curves) and in the final design (solid curves) of the RHIC dipole. The persistent-current contributions have been subtracted (R. Gupta, private communication).

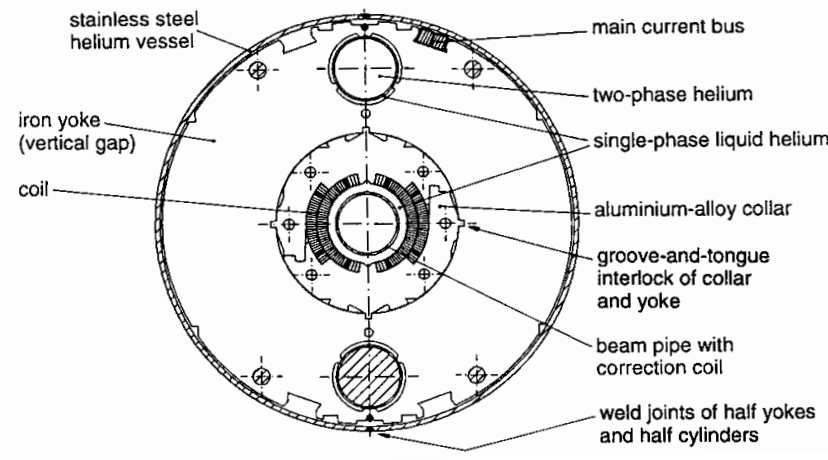

Figure 16: Cross section of the HERA dipole magnet. The coil is clamped by an aluminium-alloy collar and then surrounded by a cold-iron yoke.

\subsection{End field}

Due to the complexity of the current distribution the field calculation at the coil end can only be done numerically. For long magnets the end fields play a minor role but the perturbations may be strong enough to require a compensation in the straight section. A simple coil end without spacers between the windings yields a large negative sextupole field. In the Tevatron dipoles this endfield sextupole is compensated by a purposely introduced positive sextupole in the straight section. In more recent designs the windings in the coil head are spread out by

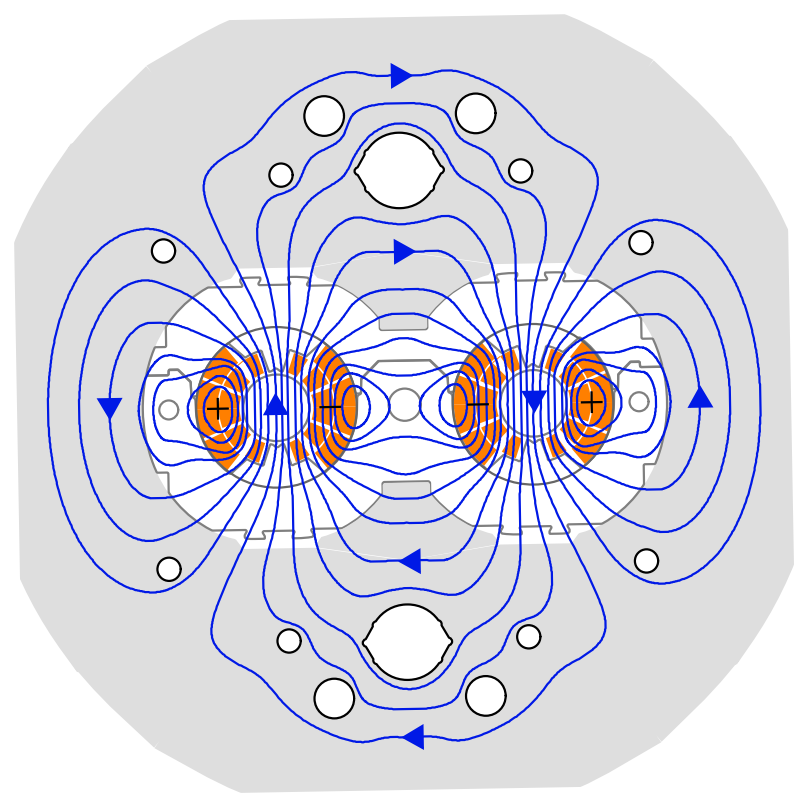

Figure 17: A cross section of the twin-aperture LHC dipole with computed field lines in the iron. One can see very clearly that the field pattern is influenced by the arrangement and size of the holes in the iron yoke. For the RHIC and LHC magnets great care was taken to optimize the hole pattern in the yoke for minimum field distortions. This cannot be done analytically but needs numerical optimization codes. (Courtesy S. Russenschuck).

epoxy-fibreglass spacers. This is shown schematically in Fig. 18a. With a suitable choice of spacers the sextupole produced by the coil ends has both positive and negative values (Fig. 18b) and averages to zero. The spacers have the additional benefit of preventing local field enhancements.

\section{Layout and Properties of Prac- tical Accelerator Magnets}

\subsection{Superconducting cable}

Of the large variety of superconducting alloys and compounds only two materials are commercially available for large scale magnet production, niobium-titanium NbTi and niobium-tin $\mathrm{Nb}_{3} \mathrm{Sn}$. The 'work horse' is niobiumtitanium in spite of the fact that its upper critical field is only $10 \mathrm{~T}$ at $4.2 \mathrm{~K}$. The outstanding feature of $\mathrm{NbTi}$ is its extreme ductility which permits effective and simple fabrication methods for wires and cables. For this reason it is widely used in magnets of moderate field strength (up to $6.5 \mathrm{~T}$ at $4.2 \mathrm{~K}$ ). Cooling with superfluid helium of $2 \mathrm{~K}$ increases the field level to about $9 \mathrm{~T}$. The optimum titanium proportion in the alloy is $46.5 \%$ (by weight). For higher fields $\mathrm{Nb}_{3} \mathrm{Sn}$ with an upper critical fields of about $18 \mathrm{~T}$ at $4.2 \mathrm{~K}$ is used. Niobium-tin is an intermetallic compound of well-defined stoichiome- 


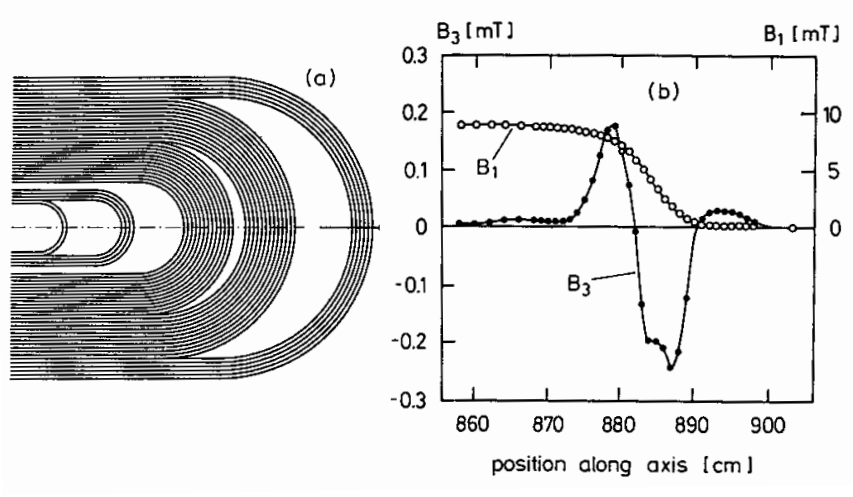

Figure 18: (a) Schematic view of coil head with spacers. (b) Measured dipole and sextupole end field of a HERA dipole for a current of $10 \mathrm{~A}$ in the normal state.

try. Because of its brittleness $\mathrm{Nb}_{3} \mathrm{Sn}$ cannot be drawn to thin filaments but must be formed in the final shape by a long heat treatment at $700^{\circ} \mathrm{C}$. Multifilamentary $\mathrm{Nb}_{3} \mathrm{Sn}$ strands are very sensitive to mechanical stress; bending with a small radius of curvature such as needed in the coil heads of accelerator magnets leads to a severe loss in current-carrying capacity.

The cables used in the coils of accelerator magnets consist of 20-40 wires (strands) of about $1 \mathrm{~mm}$ diameter. It is impossible to use wires made from pure superconductor. They would be extremely vulnerable against flux jumps during magnet excitation, the release of magnetic flux bundles from their pinning centres. Such flux jumps are accompanied with a heating of the material, often beyond the critical temperature. Since NbTi has a fairly high resistivity in the normal state the transport current would then cause a quench of the coil. Only thin superconductor filaments (diameter below $50 \mu \mathrm{m}$ for $\mathrm{NbTi}$ at $5 \mathrm{~T}$ and $4.2 \mathrm{~K}$ ) are stable against flux jumps [15]. Therefore one uses multifilamentary wires which consist of a large number of thin filaments embedded in a copper matrix which provides mechanical stability and serves as an electrical bypass of high conductivity. Cross sections of two typical strands are shown in Fig. 19.

If a filament should be heated beyond the critical temperature, for instance by a small flux jump, the current is taken over by the copper, allowing the NbTi to cool down and recover superconductivity. In order to fulfil these tasks the copper must be in as good an electrical and thermal contact with the superconductor as possible. High purity copper with excellent electrical and thermal conductivity at $4.2 \mathrm{~K}$ is needed. The so-called residual resistivity ratio $R R R$, the ratio of the resistivities at 300 $\mathrm{K}$ and $10 \mathrm{~K}$, should exceed 100 .

The most stringent constraint on the filament size derives from the field distortions due to persistent magnetization currents which are proportional to the filament diameter. With present technology, NbTi filament diameters of 5-6 $\mu \mathrm{m}$ are an optimum. A further reduction is costly and will eventually lead into the wrong direc-
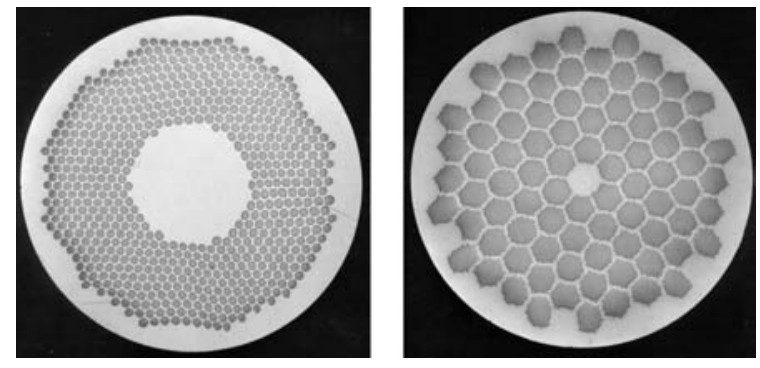

Figure 19: Cross sections of two NbTi multifilamentary strands made by Vacuumschmelze. Left side: strand used in the HERA quadrupoles, 636 filaments of $19 \mu \mathrm{m}$ diameter. Right side: prototype strand for an LHC dipole with 10164 filaments of $5 \mu \mathrm{m}$ diameter, made by a two-stage extrusion and stacking procedure. (Courtesy H. Krauth).

tion because at very small inter-filament spacings a proximity coupling occurs, basically a quantum-mechanical tunnelling of Cooper pairs through the normal material between adjacent filaments. In the coupling regime the superconductor magnetization grows with decreasing filament diameter.

The filaments in a strand are twisted with a pitch of typically $25 \mathrm{~mm}$ to suppress induced currents between different filaments during a field sweep. Also the strands in the cable are transposed with a pitch length of about $100 \mathrm{~mm}$. The ramp rate in proton storage rings is small, for instance $0.01 \mathrm{~T} / \mathrm{s}$ in HERA. In this case it is unnecessary to insulate the strands in the cable against each other. In the Tevatron magnets, every second strand is covered with an oxide layer to permit higher ramp rates.

Most superconducting accelerator magnets are made from a multi-strand cable of the so-called Rutherford type, sketched in Fig. 20. The wires are twisted and compressed into a flat two-layer cable. The cable insulation is permeable to liquid helium so the surface of all strands is wetted with the coolant. Owing to its high heat capacity the helium acts as a heat sink and stabilizes the conductor in case of transient heat production caused by wire motion.
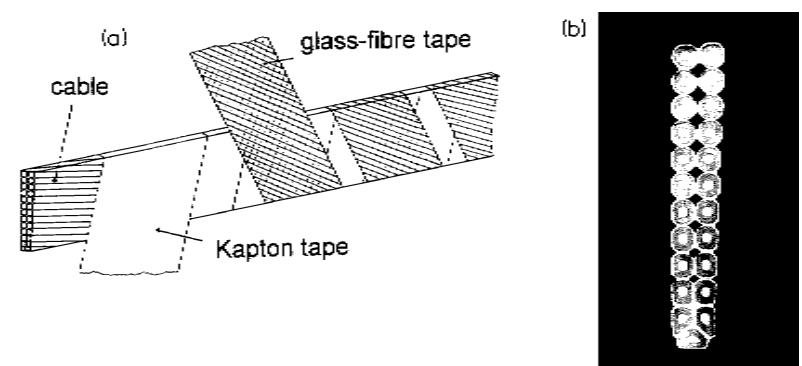

Figure 20: (a) Rutherford cable with Kapton and glass-fibre insulation such as used in the Tevatron and HERA magnets. The glass-fibre tape is pre-impregnated with B-stage epoxy which cures at temperatures above $120^{\circ} \mathrm{C}$. In the RHIC and $\mathrm{LHC}$ coils the second insulation layer is also made from Kapton. (b) Cross section of the cable. 
Ideally the cable should have a trapezoidal cross section matching an appropriate azimuthal subdivision of the current shells. This is technically possible for magnets with an inner diameter of $75 \mathrm{~mm}$ or more (Tevatron, HERA, RHIC) but meets some difficulties at smaller diameters (LHC). The cable design has to ensure tight packing of the strands to prevent wire motion during excitation.

\subsection{Coil production}

The superconducting coil is the most critical component of a magnet and a sound design is the prerequisite for achieving a high field level. The presently favoured design has evolved over the past 25 years. The basic principles stem from the dipoles and quadrupoles of the Tevatron. The demanding task of fabricating long magnets with cross sectional accuracies in the order of a few hundredths of a millimetre was first solved at Fermilab with the introduction of laminated tooling. The basic idea is that the coil has to conform with such tight tolerances at any cross section whereas in longitudinal direction the requirements are more relaxed. Attempts to produce solid mandrels and moulds for coil winding and curing by standard machining techniques turned out too costly and did not comply with the required precision. Precise laminations can be punched at moderate cost and are then assembled to long units of tooling.

Here I shortly describe the fabrication of the half coils of the HERA dipoles. The winding mandrel is stacked from punched steel laminations providing a geometrical accuracy of $0.02 \mathrm{~mm}$ at any coil cross section. The Rutherford cable is wound with an electronically controlled tension of 200 N. First, the inner half-coil comprising 32 turns is wound. It is covered with a mould, transferred to a hydraulic press and heated to $90^{\circ} \mathrm{C}$. When the B-stage epoxy in the glass-fibre insulation has become soft the coil is compressed to the required shape and afterwards cured at $160^{\circ} \mathrm{C}$. The assembly is shown in Fig. 21. The outer half-coil with 20 turns is wound on top of the cured inner half-coil and then the whole assembly is baked out again. A 100-mm-long solder joint serves as an electrical connection. The resistance of about $10^{-9} \Omega$ is sufficiently low that the produced heat is easily conducted away by the liquid helium. The inner and outer coil are separated by a $0.5 \mathrm{~mm}$ thick epoxyfibreglass layer with slots for helium passage.

The two half coils are put together and surrounded with clamps or 'collars' which provide the precise coil geometry and, most importantly, the large pre-stress in the coil needed for good performance at high field. Also the collars are assembled from precision-stamped laminations. The material is aluminium alloy or stainlesssteel (316 LN, Nitronic 40 or DIN 1.4429). Care must be taken that material preserves its low magnetic susceptibility when cooled to liquid-helium temperature.

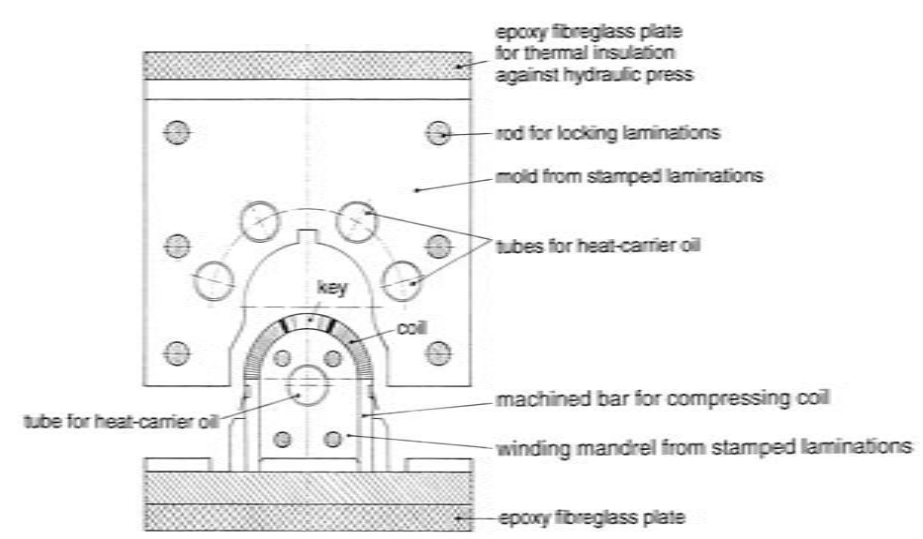

Figure 21: The inner half coil of a HERA dipole after compression and curing. For clarity the curing mould has been shifted upwards.

\subsection{Mechanical accuracies}

In Sect. 3 we have shown that the ideal $\cos \phi$ current distribution of a dipole can be approximated by using two coil layers with properly adjusted limiting angles and by spreading the current distribution with the help of longitudinal wedges. Theoretical multipole coefficients below $10^{-4}$ are easy to achieve. There is not much use to strive for smaller harmonics since in real magnets the manufacturing tolerances readily outweigh these theoretical numbers. For a single-layer dipole coil whose field is easily computed analytically we want to discuss two typical geometrical errors and evaluate their influence on field quality. If the limiting angle $\phi_{l}$ of the current shell differs from $60^{\circ}$ the sextupole coefficient is no longer zero. It can be computed from Eq. (35)

$$
b_{3}=\frac{1}{3}\left(\frac{r_{0}}{a}\right)^{2} \frac{\sin \left(180^{\circ}+3 \delta \phi_{l}\right)}{\sin \left(60^{\circ}+\delta \phi_{l}\right)}
$$

where $\delta \phi_{l}$ is the angular error. The condition that $\left|b_{3}\right| \leq 1 \cdot 10^{-4}$ requires $\delta \phi_{l} \leq 0.25 \mathrm{mrad}$, i.e. the arc length of a half coil must be accurate to $10 \mu \mathrm{m}$ for an average coil radius of $40 \mathrm{~mm}$. The position of the mid-plane between the top and bottom half coil of a magnet may move as a consequence of fabrication tolerances. During assembly of the clamps under high pressure the half-coils are compressed like two springs. The mid-plane adjusts itself depending on the relative size and spring constants of the half-coils. A typical displacement of $20 \mu \mathrm{m}$ produces a skew quadrupole $a_{2}=1.3 \cdot 10^{-4}$.

In the general case, various conceivable distortions of the coil geometry can be characterized by a transformation matrix which deforms an ideal coil in such a way that the matrix elements influence either only the normal multipoles $b_{n}$ or the skew poles $a_{n}$. From the measured multipole coefficients of a magnet one can then compute by matrix inversion the geometrical errors in the coil and apply appropriate corrections, for instance by shimming, that is by placing thin stainless-steel strips between the insulated coil package and the confining structure. 
From a comprehensive analysis one concludes that mechanical accuracies in the $20 \mu \mathrm{m}$ range are needed to satisfy the field homogeneity requirements. The best quality control on the coil accuracy is provided by multipole measurements. Figure 22 shows the normal and skew multipole coefficients of the HERA dipoles at a field of 4.7 T. Most of the coefficients are very small and well within the limits of $\pm 0.5 \cdot 10^{-4}$, which were used in the particle tracking programs for determining the dynamic aperture (maximum stable beam size) of the HERA storage ring. Two coefficients show a larger scattering: the normal sextupole $b_{3}$, which is particularly sensitive to slight changes in the limiting angles of the coil shells, and the skew quadrupole $a_{2}$, which may arise from an up-down asymmetry of the coil.

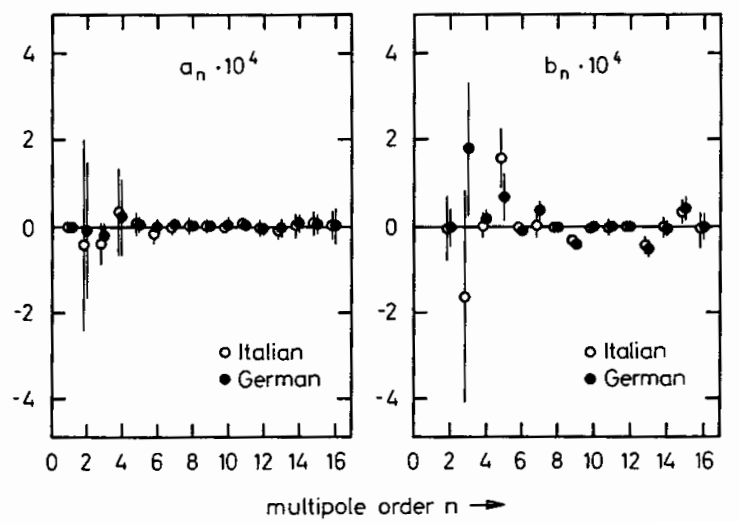

Figure 22: The normal $\left(b_{n}\right)$ and skew $\left(a_{n}\right)$ multipole coefficients of the HERA dipoles at $4.7 \mathrm{~T}$, corresponding to a proton energy of $800 \mathrm{GeV}$. Plotted are the average values with their rms standard deviations from 200 magnets of Italian and German production. The data have been averaged over the whole length of the magnets, including the coil heads. The dipoles from Italian and German production are mounted in different sections of the HERA ring to permit a compensation of the systematic sextupole by means of sextupole correction coils. Moreover, the dipoles have been sorted in the accelerator in order to minimize the effects of random sextupole variations.

\subsection{Magnetic forces}

In a dipole coil large radial and azimuthal Lorentz forces act on the superconducting cable. Summed over all windings we obtain a horizontal force of $1.5 \cdot 10^{6} \mathrm{~N}$ for a 1-m-long coil section of the HERA coil at a field of $6 \mathrm{~T}$. The clamps have to be stiff enough to sustain the huge force. Figure 23 shows the computed deformation of the aluminium-collared HERA dipole coil in two states: at room temperature and zero field and in the superconducting state at 6 Tesla. Close to the limiting angles of the coil shells, the forces are mainly azimuthal and directed towards the median plane. To prevent a motion of the coil, which would usually be accompanied with frictional heating, the coil package is mechanically prestressed by a such a high pressure (60 $\mathrm{MPa}$ or more) that the highest magnetic force per unit area is exceeded. The high prestress is a potential danger for the Kapton insulation which starts to yield at about $70 \mathrm{MPa}$ at room temperature. However, more than a decade of experience at the Tevatron and HERA has shown that the insulation is stable if the magnets are kept at 4 Kelvin most of the time.
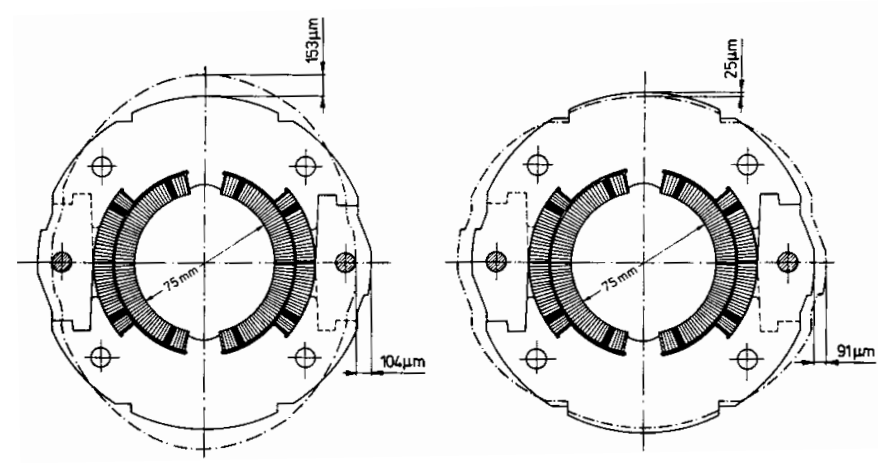

Figure 23: Calculated deformation of the collared HERA coil. Left side: room temperature, vertical deformation of collar by the precompressed coil. Right side: superconducting state, horizontal deformation of collar by the magnetic forces at $6 \mathrm{~T}$. The collar material is aluminium alloy $\mathrm{Al} \mathrm{Mg4.5 \textrm {Mn }}$ with a yield strength of $350 \mathrm{MPa}$.

\subsection{Quenches and magnet protection}

\subsubsection{Quench origins}

Basically one can distinguish two types of quenches. A 'natural' quench occurs when the critical surface of the superconductor is crossed. This happens in magnet tests where quenches are initiated on purpose by raising current and field simultaneously until the critical values are exceeded. The other type, a 'disturbance' quench, may occur with the nominal working point below the critical surface. The origin is usually a local overheating beyond the critical temperature. One reason can be heating by beam loss in an accelerator, another one conductor motion under the influence of the magnetic forces. At typical operating conditions, the critical temperature of $\mathrm{NbTi}$ is significantly lower than $9.2 \mathrm{~K}$, for instance $T_{c}(B, J) \approx 5 \mathrm{~K}$ at $B=5 \mathrm{~T}$ and $J=2000 \mathrm{~A} / \mathrm{mm}^{2}$. The heat capacity of metals is extremely small in the $4 \mathrm{~K}$ regime. An energy input of a few milli-Joules per $\mathrm{cm}^{3}$ is sufficient to raise the temperature of the conductor beyond $T_{c}$ if no helium cooling is present. This tiny energy corresponds to the work done by the Lorentz force if the conductor moves by just a few $\mu \mathrm{m}$. This illustrates how small the safety margin against quenches is. Conductor motion under the action of the huge magnetic forces can only be prevented if the coil is manufactured with high precision and clamped with a large pre-stress. 


\subsubsection{Stability}

The stability of a coil is a measure of its ability to recover superconductivity after a disturbance. We can distinguish two limiting cases:

- each wire is surrounded with liquid helium so that produced heat is directly transferred to the coolant;

- the wires are embedded in an insulating medium like epoxy and cooling of a hot spot is only possible via heat conduction along the wire.

In accelerator magnets the first mechanism dominates but heat conduction plays also a role.

We consider now a multifilamentary conductor of radius $r$ immersed in a liquid helium bath of temperature $T_{0}$, and we assume that the wire carries a current just above the critical current at $T_{0}$. Then the NbTi filaments become slightly resistive and the current is shared between the NbTi and the copper. The conductor temperature rises to $T>T_{0}$. The heat generation per unit length is found to be [16]

$$
G=\rho_{C u} J_{c}^{2} \frac{\eta^{2}}{(1-\eta)} \cdot \frac{T-T_{0}}{T_{c}-T_{0}} \cdot \pi r^{2}
$$

where $\rho_{C u}$ is the resistivity of the copper matrix and $\eta$ the fraction of superconductor in the strand. The heat flow to the helium bath is

$$
Q=2 \pi r h\left(T-T_{0}\right)
$$

where $h$ is the heat transfer coefficient. Superconductivity is recovered if the power generated is less than the power removed, $G<Q$. The ratio of the two quantities is called the Stekly parameter [35]:

$$
\alpha_{S t}=\frac{G}{Q}=\frac{\eta^{2} J_{c}^{2} \rho_{C u} r}{2(1-\eta) h\left(T_{c}-T_{0}\right)} .
$$

Complete cryogenic stability is achieved for $\alpha_{S t}<1$. A coil complying with this criterion can basically not quench since the copper alone can carry the current with the available helium cooling. For a strand with NbTi filaments in a copper matrix, cryogenic stability would require a superconductor fraction $\eta$ of less than $10 \%$. The large superconducting solenoid coils in storage ring experiments are often built as cryostable magnets by cladding the Rutherford cable with high purity aluminium which features a thousandfold increase in conductivity when cooled to $4.2 \mathrm{~K}$. In accelerator magnets a much higher superconductor proportion must be chosen since otherwise the coils would become extremely bulky and expensive. So $\alpha_{S t}$ is much larger than unity (e.g. $\alpha_{S t}=22$ in the HERA dipoles) which means that these magnets are definitely not cryostable and may quench, for instance as a consequence of beam losses.

The helium inside a Rutherford-type cable has nevertheless a beneficial effect on a not fully stabilized conductor. Baynham et al. [36] initiated quenches in superconducting wires by inductive heating. For a conductor in vacuum an energy deposition of a few $\mathrm{mJ} / \mathrm{cm}^{3}$ was sufficient to produce a quench while with liquid helium around the wire the energy had to be ten times higher. The Rutherford-type cable with heliumtransparent Kapton and glass tape insulation (see Fig. 20) provides optimum cooling as each wire is surrounded by liquid helium. Moreover, the enclosed helium increases the overall heat capacity of the Rutherford cable by nearly two orders of magnitude.

\subsubsection{Quench propagation}

The stored magnetic energy in a large accelerator dipole may be in the Mega-Joule range. When the coil quenches at some localized spot, a rapid propagation of the normal zone is of great importance. The huge magnetic energy must be distributed over a sizeable fraction of the coil volume to prevent local overheating and possible destruction of the conductor ${ }^{7}$. The quench propagation velocity is therefore an important property of a superconducting coil. The quench propagates mainly along the cable; transverse propagation is impeded by the insulation and the helium contents in the cable. The longitudinal quench propagation velocity in the adiabatic limit, neglecting heat transfer to the helium surrounding the strands, is given by the expression [16]

$$
v \equiv v_{a d i a b}=\frac{J}{C} \sqrt{\frac{\rho \lambda}{T_{c}-T_{0}}} .
$$

Here the heat capacity $C$ and the longitudinal heat conductivity $\lambda$ of the cable have been treated as independent of $T$. Inserting reasonable numbers yields velocities in the order of $50-100 \mathrm{~m} / \mathrm{s}$. Extensive measurements of the propagation of heater-induced quenches were carried out on prototype dipoles for the former SSC (Superconducting Super Collider) project. Figure 24a shows that velocities of more than $200 \mathrm{~m} / \mathrm{s}$ were observed. The surprisingly high speeds near the critical current cannot be explained in terms of an adiabatic model and are probably caused by a supersonic pressure wave in the liquid helium.

\subsubsection{Heating of the coil after a quench}

We have seen that economic and spatial reasons preclude the use of a cryostable conductor in accelerator magnets. It is then mandatory to investigate how much the coil may heat up after a quench. A conservative limit is $100 \mathrm{~K}$ because then the thermal expansion is very small and mechanical stress in the coil and support structure is avoided. Common practice is to admit heating well beyond room temperature, and the experience with hundreds of magnets has shown that this causes no damage.

\footnotetext{
${ }^{7}$ In laboratory magnet tests one extracts most of the stored energy and dissipates it in an external dump resistor. This is not possible in the accelerator where many magnets are connected in series. Here each coil has to be able to absorb its own field energy.
} 

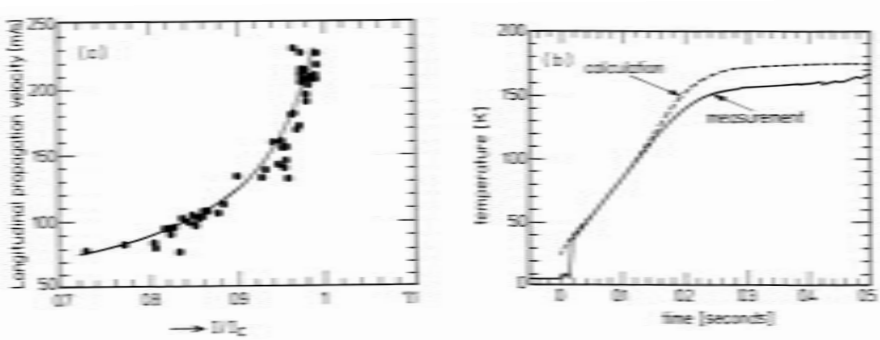

Figure 24: (a) Longitudinal quench propagation velocity in SSC dipoles as a function of $I / I_{c}$. (b) Time evolution of the hot-spot temperature during a quench induced at 6500 A on turn 1 of the inner coil of an SSC dipole (Courtesy A. Devred).

Once a quench has been detected the power supply is switched off and the stored magnet energy is dissipated in a dump resistor and in the normal-conducting part of the coil. The current decays with a typical time constant of a few $100 \mathrm{~ms}$. A relation can be established between the time dependence of the current after a quench and the highest temperature in the coil. The power density in a normal coil section is $\rho(T) J^{2}(t)$. During a time interval $d t$ the section is heated by $d T=\frac{\rho(T)}{C(T)} J^{2}(t) d t$. Separation of variables and integration yields

$$
\int_{0}^{\infty} J^{2}(t) d t=\int_{T_{0}}^{T_{\max }} \frac{C(T)}{\rho(T)} d T=F\left(T_{\max }\right) .
$$

From the temperature-dependent material properties the integral on the right hand side can be evaluated as a function of the maximum temperature $T_{\max }$. The integral on the left hand side can be measured. This way it is possible to establish a relation between the time integral of $J^{2}$ and the so-called hot spot temperature $T_{\text {max }}$, the highest temperature in the coil. The temperature rise of a quenched coil section is plotted in Fig. 24b as a function of time after the quench has been detected and the power supply switched off. Within $200 \mathrm{~ms}$ the temperature reaches a plateau of $150 \mathrm{~K}$. The data agree well with model calculations.

\subsubsection{Protection of a string of magnets}

An accelerator contains a large number of magnets which are connected in series and constitute a large inductance (25 Henry in HERA). Magnet protection is a serious challenge since it is impossible to discharge this large inductance fast enough that overheating of a quenched coil is avoided. If one tried to extract the stored magnetic energy via a single external resistor within a few hundred milliseconds the induced voltage would exceed $250 \mathrm{kV}$ and cause spark discharges all around the ring. The solution is to reduce the current in the string slowly but guide it around a quenched magnet by means of a thyristor (Tevatron) or a diode (HERA, RHIC, LHC). Cottingham [37] first proposed to mount silicon diodes inside the liquid helium cryostat. This concept has proven very reliable.

\subsubsection{Quench performance of practical magnets}

It cannot be taken for granted that superconducting magnets reach the upper critical field of the superconductor. Historically, premature quenching has been quite common, see [15] for a discussion. Accelerator magnets can fortunately be built so well that the critical field is achieved with little if any 'training'. There are two prerequisites for such an optimal performance:

- the Rutherford-type cable features excellent mechanical stability with good fixation of the strands, and the Kapton insulation is transparent to liquid helium,

- the clamps provide a mechanical compression of the coil in excess of the Lorentz forces such that motion of a winding turn is inhibited.

The RHIC dipoles exhibited very little training and feature an ample safety margin. The same applies for the HERA magnets. All dipoles passed 5 Tesla at the first attempt, and for the majority one or two steps sufficed to arrive at the critical field of $6 \mathrm{~T}$ (the test temperature was $4.75 \mathrm{~K}$ ).

The LHC magnets with a design field of $8.4 \mathrm{~T}$ enter a new regime. The magnetic forces are a factor af two larger than in 5-6 T magnets and important structural materials like Kapton or even the soft copper matrix in the cable are close to their plastic limit. Nevertheless, the quench performance achieved with prototypes leaves no doubt that dipoles of the LHC type can be operated reliably and with sufficient safety margin.

\section{Persistent Magnetization Cur- rents}

\subsection{Superconductor magnetization}

Persistent magnetization currents in the superconductor filaments are the source of severe field distortions at low excitation of a superconducting accelerator magnet. These bipolar currents generate all multipoles which are allowed by coil symmetry: $b_{1}, b_{3}, b_{5}, b_{7}, \ldots$ in a dipole, $b_{2}, b_{6}, b_{10}, b_{14}, \ldots$ in a quadrupole. A distinct hysteresis behaviour is observed: the multipole fields are of opposite sign for increasing and decreasing main field, respectively.

We can distinguish three types of bipolar currents which are induced in the coil by the time-varying magnetic field: eddy currents between different strands in the cable, coupling currents between different filaments inside a strand, and finally the magnetization currents inside individual filaments. To suppress the eddy and 
coupling currents the strands in the cable are transposed with a pitch length of about $100 \mathrm{~mm}$ and the filaments in the strands are twisted with a twist length of typically $25 \mathrm{~mm}$. These currents decay exponentially with typical time constants in the order of a second or less. It should be mentioned, however, that there exist so-called 'boundary-induced' currents with life times of many hours [38], see sect. 6.4.

Truly persistent currents occur only within individual filaments. Their computation is based on the 'critical state model' [39] according to which a hard superconductor tries to shield itself from any external field variation by generating a bipolar current distribution with the highest possible density, namely the critical current density. $J_{c}(B, T)$ depends on the local field in the coil and on temperature. Let us study the response of a superconductor filament to a homogeneous external field $B_{\text {ext }}$ which is first raised from zero and then lowered again. With increasing $B_{\text {ext }}$, a $\cos \phi$-like current distribution is induced (Fig. 25a) producing a homogeneous shielding field $B_{\text {shield }}$ which just cancels $B_{\text {ext }}$ in the current-free region of the filament. Following Wilson [15] we approximate the boundary of the current-free region by an ellipse with large half axis $a=r_{f}$ (filament radius), small half axis $b$ and eccentricity $\varepsilon=\sqrt{1-(b / a)^{2}}$. The shielding field can be computed analytically (see e.g. [16] )

$$
B_{\text {shield }}=-\frac{2 \mu_{0} J_{c} r_{f}}{\pi}\left(1-\sqrt{1-\varepsilon^{2}} \cdot \frac{\arcsin \varepsilon}{\varepsilon}\right) .
$$

The highest field which can be shielded from the interior of the filament is called the 'penetrating' field $B_{p}=2 \mu_{0} J_{c} r_{f} / \pi$ and is obtained for an ellipse shrunk to a line, i.e. $\varepsilon=1$. Figure $25 \mathrm{~b}$ shows the currents in the 'fully penetrated' filament. The applied field may be raised to much larger values than $B_{p}$ which is only about $0.13 \mathrm{~T}$ for the HERA conductor. In that case the same current pattern is obtained as in Fig. 25b but with a non-vanishing field throughout the filament. If now the field is decreased again, persistent currents with opposite polarity are superimposed because again the superconductor tries to avoid a change of its inner field. A more complicated current pattern arises as indicated in Fig. 25c. The current loops are assumed to be closed at the coil ends.

The magnetization of the NbTi filament, defined as the magnetic moment per unit volume, is given by [16]

$$
M=-\frac{4}{3 \pi} J_{c} r_{f} \varepsilon^{2}
$$

It assumes its peak value $M_{p}=|M|_{\max }=4 J_{c} r_{f} /(3 \pi)$ for the fully penetrated filament (Fig. 25b). Note that $M_{p}$ is not a constant but decreases proportional to the critical current density $J_{c}(B, T)$ when the external field is raised beyond the penetrating field. The field-generating transport current density $J_{t}$ in the superconducting coil reduces the filament area available for the magnetization currents by the factor $\left(1-\left(J_{t} / J_{c}\right)^{2}\right)$. This correction is negligible near the injection field where $J_{t} \ll J_{c}$ but becomes significant at high excitation of the magnet.

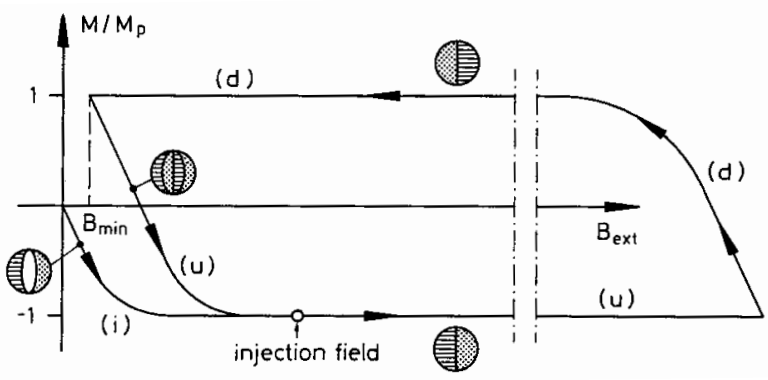

Figure 26: The normalized magnetization $M / M_{p}$ of a $N b T i$ filament as a function of the external field. (i): initial curve, (u): up-ramp branch, (d): down-ramp branch. Also shown are the current distributions in the filament. The field dependence of $J_{c}$ has been neglected.

The magnetization as a function of the external field is plotted schematically in Fig. 26. We observerve a hysteresis with three different states: Starting at the virgin state the magnetization follows an initial curve (i) and reaches its peak value at $B_{\text {ext }}=B_{p}$. After going up to high field the ramp direction is reversed and $M$ follows the 'down-ramp' branch (d). At a certain minimum current the field is increased again and the magnetization follows the 'up-ramp' branch (u) which has the remarkable feature that $M$ changes its sign from positive to negative values. This is exactly what is observed in the 6 -pole and 12-pole coefficients (see Fig. 28 below). In Fig. 26 also the current pattern in the filament is indicated at different positions of the hysteresis loop.

\subsection{Calculation of persistent-current multipole fields and comparison with data}

The field distortions from persistent currents have been computed by various authors $[40,41,42]$. In the following we describe a program [43] developed at DESY to model the persistent-current multipoles of the HERA magnets with high accuracy. We start with four symmetrically arranged filaments in the dipole coil in which bipolar currents have been induced by the increasing main field (see Fig. 27a). The current distribution in each filament can be replaced by a pair of line currents $+I$ and $-I$ whose strength equals the integrated current density and whose separation $d$ is adjusted to yield the computed filament magnetization (the result is $d \approx r_{f}$ ). Since $d \ll R$ the vector potential of the four current pairs in Fig. 27b can be derived by first-order Taylor expansion from the vector potential $A$ of four single currents (use Eq. (33) with $a$ replaced by $R$ ):

$$
A^{\text {pair }}=\frac{\partial A}{\partial R} \Delta R+\frac{\partial A}{\partial \phi} \Delta \phi
$$




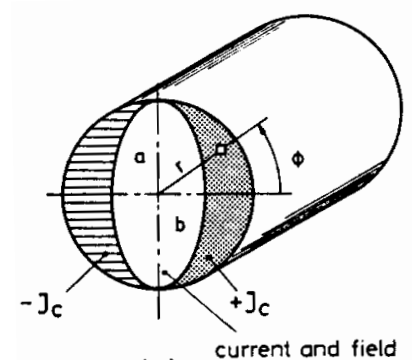

(a)

tree region

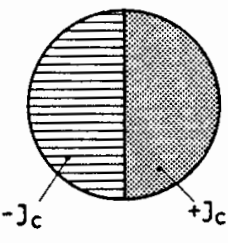

(b)

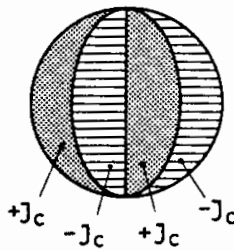

(c)

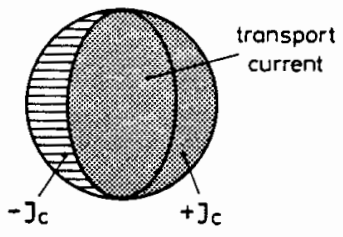

(d)

Figure 25: Schematic view of the persistent currents which are induced in a superconducting filament by a varying external field. (a) The external field is raised from zero to a value $B_{e x t}$ less than the penetrating field $B_{p}$. (b) $A$ 'fully-penetrated' filament, i.e. $B_{e x t} \geq B_{p}$. (c) Current distribution which results when the external field is first increased from zero to a value above $B_{p}$ and then decreased again. (d) Same as (b) but with a large transport current.
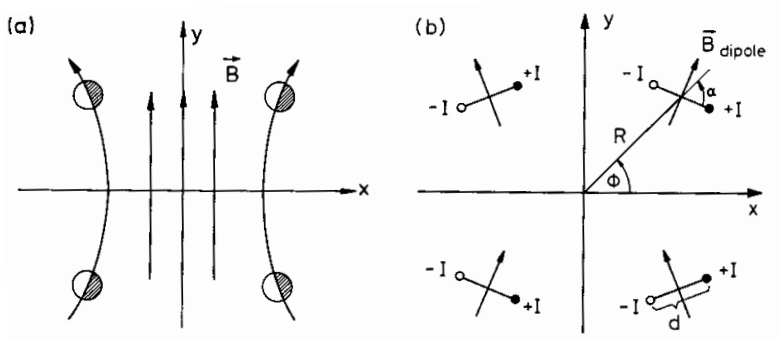

Figure 27: (a) Magnetization currents induced by the timevarying main field in four symmetrically arranged filaments inside the dipole coil. (b) Equivalent pairs of line currents. The separation $d$ between the positive and negative currents is grossly exaggerated.

With the relations $\Delta R=d \cos \alpha, \Delta \phi=-d \sin \alpha / R$ we obtain

$$
A^{\text {pair }}(r, \theta)=C \sum_{n=1,3, \ldots}\left(\frac{r}{R}\right)^{n} \cos (n \theta) \cos (n \phi+\alpha) .
$$

with $C=-2 \mu_{0}(I \cdot d) /(\pi R)$. For the product $(I \cdot d)$ we insert the magnetic moment of the filament per unit length $\pi r_{f}^{2} M=-4 J_{c} \varepsilon^{2} r_{f}^{3} / 3$. Expression (49) has to be summed over all NbTi filaments in one quarter of the dipole coil and divided by the main dipole field to obtain the multipole coefficients.

With Eq. (49) we have derived a remarkable result: the bipolar persistent currents generate exactly the same 'allowed' multipoles as the unidirectional transport current, namely the normal multipoles $b_{n}$ of the orders $n=1,3,5, \ldots$. The even orders $n=2,4, \ldots$ are absent and so are the skew multipoles $a_{n}$. 'Unallowed' poles can only be present when the superconductor properties, for instance the critical current density, are not uniform over the coil cross section. An important ingredient to the model is the critical current density $J_{c}(B, T)$ at low fields which is derived from magnetization measurements with an uncertainty of about $10 \%$. This is the dominant error in the calculation of persistent-current multipoles, the filament diameter is known to about $5 \%$.
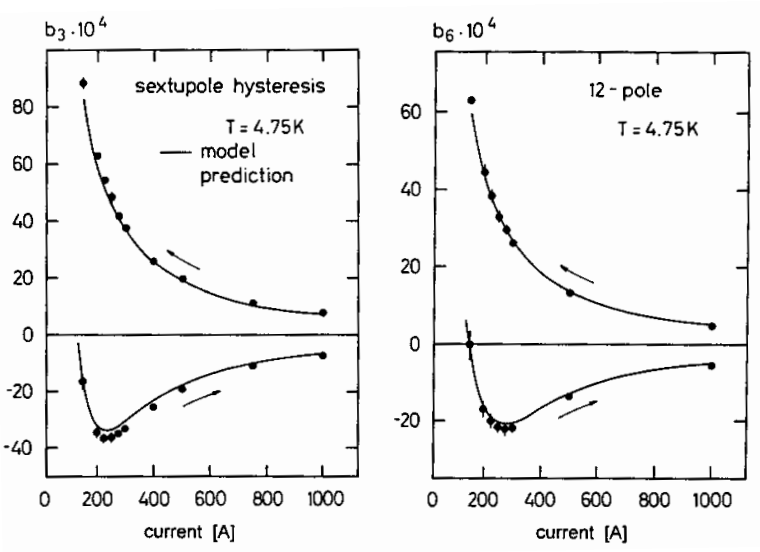

Figure 28: The averaged sextupole coefficients $b_{3}$ with rms errors from 315 HERA dipoles (12-pole coefficients $b_{6}$ from 236 quadrupoles) as a function of coil current. The curves represent absolute model predictions. The ramp direction of the current is indicated by arrows. Before starting the measurements, a current cycle $50 \mathrm{~A} \rightarrow 6000 \mathrm{~A} \rightarrow 50 \mathrm{~A}$ was performed to establish a well-defined initial condition for the superconductor magnetization.

The averaged sextupole data of 315 HERA dipoles and the 12-pole data of 236 quadrupoles are shown in Fig. 28 for increasing and decreasing main field. The predictions of the model, shown as continuous curves, are in excellent agreement with the measurements. Note that these are absolute predictions without adjustable parameters, the only input is the critical current density as a function of field. The persistent currents have also a significant influence on the main dipole field and quadrupole gradient. Again a hysteresis is observed and the data are in good agreement with the model prediction. At the HERA injection energy the main dipole field (quadrupole gradient) is $0.5 \%(0.2 \%)$ lower than the value computed from the coil current. Of course a correction is needed to match HERA to the energy of the pre-accelerator. 
The superconductor magnetization and the resulting multipoles are proportional to the filament diameter. When the HERA superconductor was specified in 1984, an optimization of costs and critical current resulted in a filament diameter above $10 \mu \mathrm{m}$. In recent years great progress has been made towards finer filaments. In the LHC magnets, the diameter is $6 \mu \mathrm{m}$.

\subsection{Time dependence of persistent- current effects}

Since the magnetization currents flow entirely within the superconducting filaments it came as a total surprise when a time dependence of the sextupole component was observed in the Tevatron dipoles [44]. The data could not be described in terms of a single exponential but required two or more time constants. Similar observations were made on HERA magnets and it was found that the multipole fields are much better described by a logarithmic instead of an exponential time dependence. As an example we show in Fig. 29a the time dependence of the sextupole coefficient in a dipole magnet. The drift is well represented by a function of the form $A-R \log t$. A curious observation is that the 'decay rate' $R$, measured at low field, depends strongly on the previous excitation level of the magnet.

A logarithmic time dependence in hard superconductors was first observed in 1962 and attributed to thermally-activated flux creep [45], [46]. This flux creep is indeed visible in a sample of the HERA conductor, see Fig. 29b, but in contrast to the magnet measurement the logarithmic time variation of the cable magnetization is comparatively small and totally independent of the preceding field cycle. From the data in Fig. 29 it is obvious
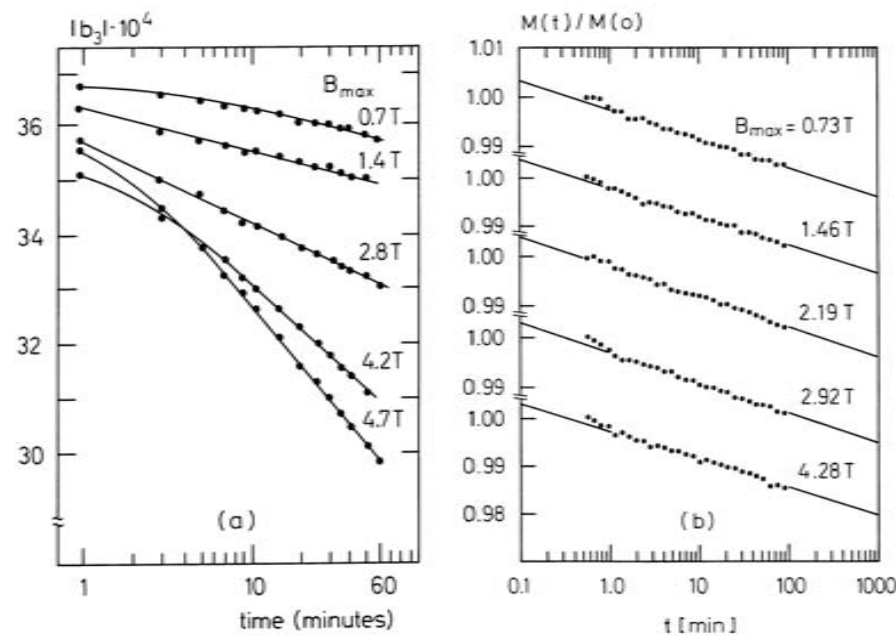

Figure 29: (a) Decay of the sextupole in a HERA dipole at a field of $0.23 \mathrm{~T}$ for different values of the maximum field in the initializing cycle $0 \rightarrow B_{\max } \rightarrow 0.04 \mathrm{~T} \rightarrow 0.23 \mathrm{~T}$ [47]. (b) Magnetization decay at zero field in a long sample of HERA cable for different values of the maximum field in the initializing cycle $0 \rightarrow B_{\max } \rightarrow 0$ [48]. that thermally activated flux creep can explain only part of the time dependence of multipoles in magnets. The decay rates measured in magnets are usually much larger than those in cable samples, and there is a considerable variation from magnet to magnet. In 1995 experimental results [38] and model calculations [49] were presented showing that the time dependence is due to a complex interplay between magnetization currents in the NbTi filaments and eddy currents among the strands of the cable. Quantitative predictions are not possible because of too many unknown parameters. For a more detailed discussion see Sect. 6 .

\subsection{Longitudinal periodicity of multipole fields}

In a measurement of the time-varying persistent-current sextupole at various positions in a HERA dipole the surprising discovery was made [50] that the sextupole field exhibits a pronounced periodic pattern along the axis of the magnet. Similar observations were subsequently made in other magnets.

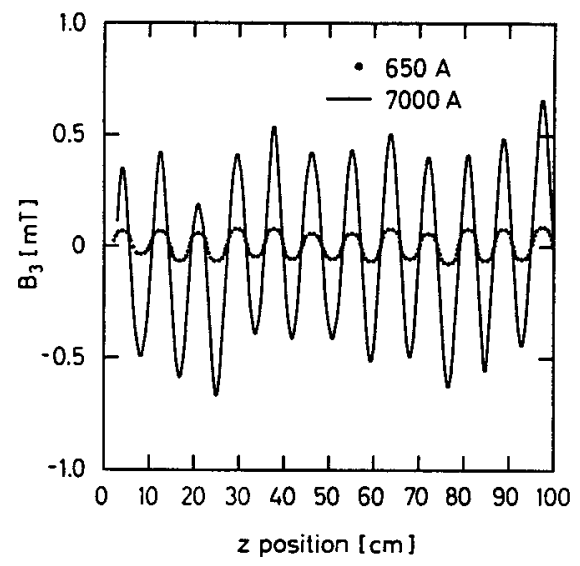

(a)
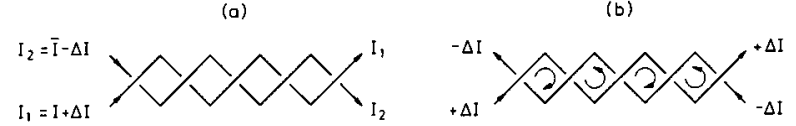

Figure 30: Top: Longitudinal periodicity of the sextupole in an SSC dipole at $650 \mathrm{~A}$ and $7000 \mathrm{~A}$ [51] (C) 1993 IEEE). Bottom: (a) Simplest model for the generation of longitudinally periodic field perturbations. A two-strand Rutherford cable is considered in which one stand carries more current than the other. (b) Subtracting the average transport current, a bipolar current pattern is obtained which is equivalent to a periodic sequence of alternating magnetic moments.

The oscillation is particularly pronounced when the magnet has been excited to high field, as can be seen from Fig. 30, where the sextupole in a prototype dipole for the terminated SSC project is plotted for magnet currents of 650 and $7000 \mathrm{~A}$ [51]. At $7000 \mathrm{~A}$ the oscillation amplitude is much larger than the average value of the sextupole field. An interesting observation is that the 
large oscillation amplitude persists when the current is afterwards reduced to a low value.
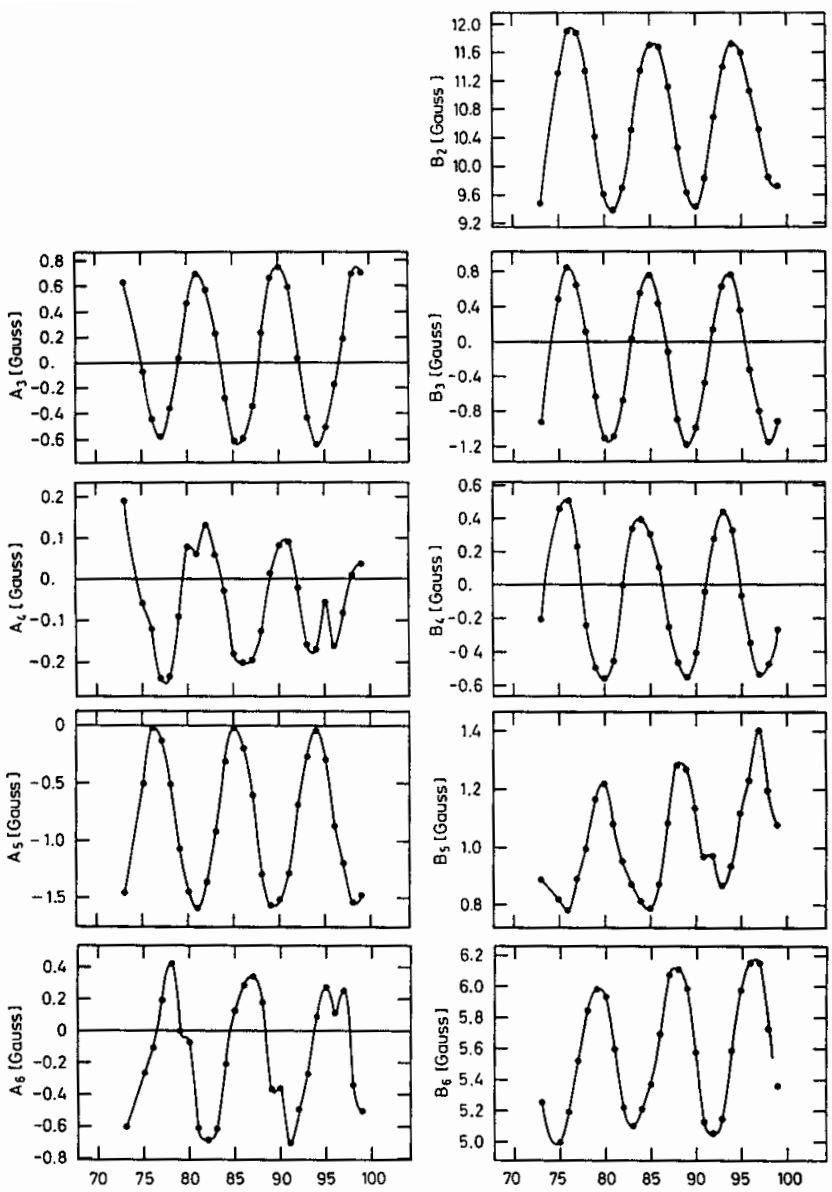

position along quadrupole axis $[\mathrm{cm}]$

Figure 31: Periodicity of the skew $\left(A_{n}\right)$ and normal $\left(B_{n}\right)$ multipole fields at $r_{0}=25 \mathrm{~mm}$ in a HERA quadrupole. The data were taken at coil current $I=0$ after a current cycle 0 $\rightarrow 6000 A \rightarrow 0$ had been perfomed. Note the large average values of the allowed multipole fields $B_{2}$ and $B_{6}$. Remark for experts: The skew quadrupole field $A_{2}$ could not be measured since the rotating pick-up coil system was not equipped with an absolute angle sensor.

In Fig. 31 we show data from a HERA quadrupole taken with a $20-\mathrm{mm}$-long rotating pick-up coil. Not only the allowed multipole fields $B_{2}, B_{6}, \ldots$ are found to be modulated but all $A_{n}$ and $B_{n}$ exhibit the periodic pattern. This proves that the currents responsible for this pattern do not obey any coil symmetries. Note, however, that the unallowed multipoles average to zero while the average values of the allowed multipoles agree with the persistent-current induced multipole fields.

A clue to an understanding of the longitudinal periodicity is the observation that the wavelength of $94 \mathrm{~mm}$ in the HERA magnets agrees with the transposition pitch of $95 \pm 2 \mathrm{~mm}$ in the Rutherford cable. These findings were confirmed at Brookhaven and CERN. The periodic pat- tern can be qualitatively explained by assuming a current imbalance between the strands of the cable. The simplest model is that of a two-strand cable in which one wire carries a higher current than the other. This is sketched schematically in Fig. 30. The resulting zig-zag pattern of currents leads to a sequence of alternating magnetic moments and to an almost sinusoidal field perturbation along the axis. The origin of the current imbalance are the 'boundary-induced' currents [38] mentioned above, see Sect. 6 for further discussion.

It is worth mentioning that the oscillating multipole fields have a negligible effect on the proton beam emittance because their period is orders of magnitude smaller that the betatron oscillation wavelength.

\section{Eddy Current Effects in Super- conducting Magnets}

Most superconducting accelerator magnets are made from the Rutherford-type cable described in Sect. 4. The cable consists of 20 to 40 strands of 0.7 to $1.3 \mathrm{~mm}$ diameter which are twisted around each other and shaped into a two-layer flat cable. Usually the cable is compressed to a trapezoidal cross section and its average thickness is less than twice the strand diameter. At their cross-over points, the strands are indented and have a fairly large contact area which, in combination with the high prestress in the collared coil, leads to small inter-strand resistances of about $10 \mu \Omega$. The two layers of strands thus form an arrangement of loops in which eddy currents of sizeable strength can be induced by a time-varying magnetic field. These eddy currents between different strands will be referred to as cable eddy currents. Another type are the coupling currents between different filaments inside a strand. Additional eddy currents arise in the copper wedges of the coil, in the collars, the yoke and other conducting materials.

\subsection{Coupling currents within a strand}

The filaments inside a strand are strongly coupled through the copper matrix. An effective means to reduce the filament coupling is provided by twisting the wire. Thereby the length of the loop that is exposed to the time-varying magnetic field is considerably shortened and the contributions from adjacent loops alternate in sign. In a multifilamentary twisted superconducting wire, the eddy currents run in a zig-zag fashion along the wire. The path is partly superconductive (inside the filaments), partly normal (in the copper matrix). A very clear analysis of the problem can be found in the book by Wilson [15]. The screening currents obey a $\cos \phi$ dependence and thus produce a homogeneous shielding field $B_{\text {shield. }}$. The inner field $B_{\text {int }}=B_{\text {ext }}-B_{\text {shield }}$ is also homogeneous. In case of a constant ramp rate, the strand 
magnetization resulting from the coupling

$$
M_{\text {strand }}=2 \dot{B}_{\text {int }} \tau / \mu_{0} \text {. }
$$

Here, $\tau$ is a time constant that is related to the twist pitch length $l_{\text {twist }}$ of the strand and the effective transverse resistivity $\rho_{t}$ of the copper-NbTi composite:

$$
\tau=\frac{\mu_{0}}{2 \rho_{t}}\left(\frac{l_{\text {twist }}}{2 \pi}\right)^{2}
$$

A typical value for the twist pitch is $25 \mathrm{~mm}$, a typical time constant is $10 \mathrm{~ms}$. So the strand magnetization resulting from the intra-strand eddy currents is quite shortlived and its contribution to the field distortions during the acceleration of the particle beam is small in comparison with that of the persistent-currents. The dissipated power $P$ per unit volume is derived from the formula

$$
P d t=M_{\text {strand }} d B_{i n t}=\frac{2 \dot{B}_{i n t} \tau d B_{i n t}}{\mu_{0}}=\frac{2 \dot{B}_{i n t}^{2} \tau}{\mu_{0}} d t
$$

from which follows

$$
P=\frac{2 \dot{B}_{i n t}^{2} \tau}{\mu_{0}}
$$

The ramp time $T_{\text {ramp }}$ from zero to the maximum field is large compared to the time constant $\tau$, so $\dot{B}_{\text {int }} \approx \dot{B}_{\text {ext }}=$ $B_{\max } / T_{\text {ramp }}$. The intra-strand energy dissipation per unit volume and per ramp cycle is thus

$$
Q_{\text {strand }}=\frac{B_{\max }^{2}}{\mu_{0}} \cdot \frac{4 \tau}{T_{\text {ramp }}} .
$$

This is part of the a.c. loss of the magnet.

From the equations (50) to (53) it is obvious that an untwisted superconductor ( $l_{\text {twist }}$ typically $>1000 \mathrm{~m}$ ) would be totally useless for accelerator application.

\subsection{Cable eddy currents}

\subsubsection{One dimensional model}

Following a model originally developed by Morgan ([52] and expanded by Devred and Ogitsu [53], we replace the Rutherford cable by a two-layer network of wires that are connected by small resistors at the cross-over points. A schematic drawing of a cable with six strands is given in Fig. 32. Let $N$ be the total number of strands. Across the cable we can distinguish $N-1$ different loops, labelled by the letter $n(1 \leq n \leq N-1)$. The magnetic flux through loop $n$ is denoted by $\Phi_{n}$. Let $R_{n}$ be the resistance at the $n$th transition and $i_{n}$ the cross-over current. Neglecting a dependence on the longitudinal coordinate we get the following set of equations for the inner loops $(2 \leq n \leq N-2)$

$$
\frac{d \Phi_{n}}{d t}=2 R_{n} i_{n}-R_{n-1} i_{n-1}-R_{n+1} i_{n+1}
$$

while at the edges of the cable we have

$$
\begin{aligned}
\frac{d \Phi_{1}}{d t} & =2 R_{1} i_{1}-R_{2} i_{2}, \\
\frac{d \Phi_{N-1}}{d t} & =2 R_{N-1} i_{N-1}-R_{N-2} i_{N-2} .
\end{aligned}
$$

Let $I_{n}$ be the induced current flowing in the wire section between the cross-over points $n-1$ and $n$. From Kirchhoff's law we get

$$
I_{n}=I_{n+1}+i_{n} .
$$

The sum of all currents $I_{n}$ must vanish since the timevarying field is unable to induce a net current in the $z$ direction, hence

$$
\sum_{n=1}^{N} I_{n}=0
$$

The equations (54) to (57) lead to a set of $N-1$ independent equations for the $N-1$ unknowns $i_{n}$. The solutions are for $n=1$ resp. $2 \leq n \leq N-1$

$$
\begin{aligned}
i_{1} & =\frac{1}{N R_{1}} \sum_{m=1}^{N-1} \sum_{k=1}^{m} \frac{d \Phi_{k}}{d t}, \\
i_{n} & =\frac{1}{R_{n}}\left[n R_{1} i_{1}-\sum_{m=1}^{n-1} \sum_{k=1}^{m} \frac{d \Phi_{k}}{d t}\right] .
\end{aligned}
$$

With the simplifying assumptions that the contact resistances at the cross-over points are all identical, $R_{n}=R_{c}$, and that moreover the magnetic flux across the cable is uniform, it is easy to express the solution in closed form

$$
\begin{aligned}
I_{n} & =\frac{1}{24 R_{c}}\left[N^{3}-N-2\left(n^{2}-n\right)(3 N-2 n+1)\right] \frac{d \Phi}{d t} \\
i_{n} & =\frac{n}{2 R_{c}}(N-n) \frac{d \Phi}{d t} .
\end{aligned}
$$

Figure 32 shows schematically the resulting current pattern in our model cable with six strands. The induced branch currents flow in a zig-zag fashion with different directions in the lower and upper half of the cable. It is obvious that this bipolar current distribution represents a magnetic moment and will contribute to the multipole contents of the magnet.

The contact resistances lead of course to Ohmic heat generation in time-varying magnetic fields. If we call $l_{p}$ the transposition pitch length of the cable, the overall heat generation per metre of cable (the a.c. loss) is given by the expression

$$
G=\sum_{n=1}^{N-1} R_{c} i_{n}^{2} N / l_{p}=\frac{N}{4 R_{c} l_{p}}\left(\frac{d \Phi}{d t}\right)^{2} \frac{\left(N^{4}-1\right) N}{30}
$$

The time derivative of the flux is

$$
\frac{d \Phi}{d t} \approx \frac{w l_{p}}{N^{2}} \frac{d B}{d t}
$$

where $w$ is the cable width. For $N \gg 1$ Eq. (60) reduces to

$$
G \approx \frac{w^{2} l_{p} N^{2}}{120 R_{c}}\left(\frac{d B}{d t}\right)^{2}
$$




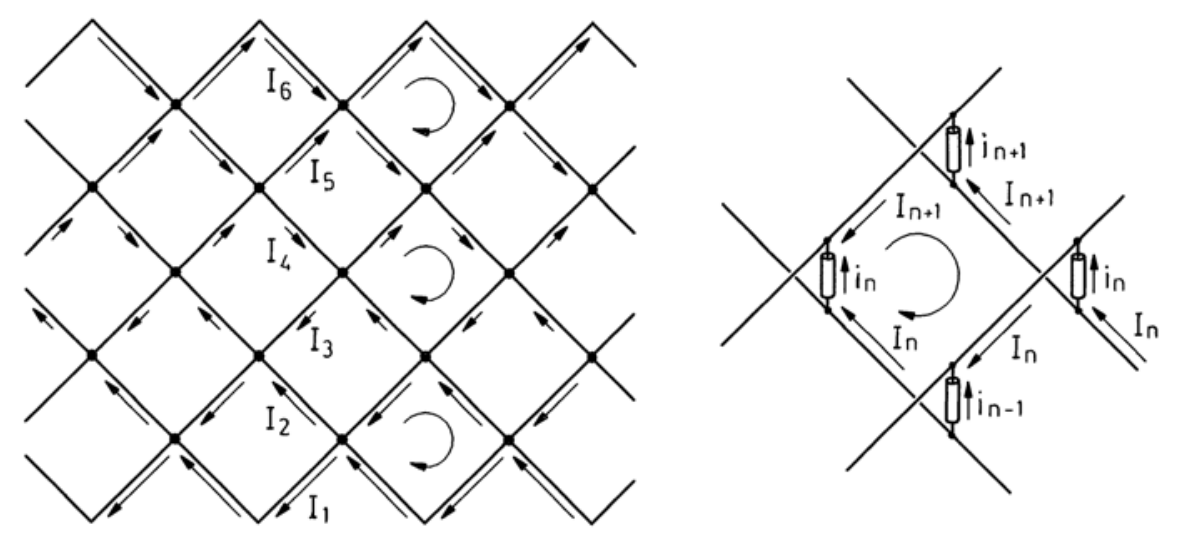

Figure 32: Equivalent resistive network of a Rutherford cable with $N=6$ strands. The cross-over resistances are indicated as black dots. Also shown are the branch currents $I_{n}$ along the strands as computed by Eq. (59). A single loop with the cross-over currents $i_{n}$ is shown on the right-hand side.

\subsubsection{Two dimensional model}

Akhmetov, Devred and Ogitsu [54] have extended the above analysis to the case that the inter-strand resistances and the branch currents are allowed to vary along the magnet axis. They subdivide the cable into columns along the axis which are labelled by an index $k$. This is sketched in Fig. 33.

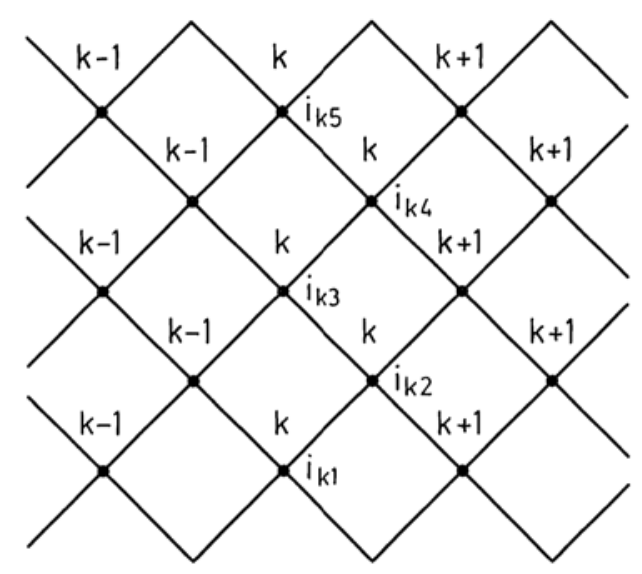

Figure 33: Resistive network with a longitudinal and transverse variation of the cross-over currents. Indicated are the 5 cross-over currents corresponding to the longitudinal index $k$.

The induced cross-over currents $i_{k, n}$ depend now on the longitudinal index $k$ and the transverse index $n$. The most general case can be treated only numerically. With the assumption of a uniform cross-over resistance both across and along the cable and a uniform magnetic flux along the cable, but allowing for a flux variation transverse to the cable (which is indeed the case in all magnet coils), one arrives at an interesting observation: the cross-over currents $i_{k, n}$ exhibit a longitudinal periodicity:

$$
i_{k+N, n}=i_{k, n} .
$$

The periodicity interval is identical with the cable twist pitch length $l_{p}$. We illustrate this again by studying the model cable with six strands. The equations (54) and (55) for the cross-over currents have to be modified to encorporate the longitudinal dependence.

$$
\begin{aligned}
i_{k, 1}+i_{k+1,1}-i_{k, 2} & =f_{1} \\
i_{k, 2}+i_{k+1,2}-i_{k+1,1}-i_{k+1,3} & =f_{2} \\
i_{k, 3}+i_{k+1,3}-i_{k, 2}-i_{k, 4} & =f_{3} \\
i_{k, 4}+i_{k+1,4}-i_{k+1,3}-i_{k+1,5} & =f_{4} \\
i_{k, 5}+i_{k+1,5}-i_{k, 4} & =f_{5} .
\end{aligned}
$$

Here we have used the abbreviation $f_{n}=\frac{1}{R_{c}} \frac{d \Phi_{n}}{d t}$. From these equations one can compute the currents at position $k+1$ from the values at position $k$. For this purpose it is convenient to use matrix notation. We define a $5 \times 5$ (generally $(N-1) \times(N-1))$ matrix $\mathbf{A}$ by

$$
\mathbf{A}=\left[\begin{array}{rrrrr}
-1 & 1 & 0 & 0 & 0 \\
-1 & 1 & -1 & 1 & 0 \\
0 & 1 & -1 & 1 & 0 \\
0 & 1 & -1 & 1 & -1 \\
0 & 0 & 0 & 1 & -1
\end{array}\right]
$$

and the vectors

$$
\mathbf{I}_{k}=\left(\begin{array}{c}
i_{k, 1} \\
i_{k, 2} \\
i_{k, 3} \\
i_{k, 4} \\
i_{k, 5}
\end{array}\right), \quad \mathbf{F}=\left(\begin{array}{c}
f_{1} \\
f_{1}+f_{2}+f_{3} \\
f_{3} \\
f_{3}+f_{4}+f_{5} \\
f_{5}
\end{array}\right)
$$

Then the equations (62) read

$$
\mathbf{I}_{k+1}=\mathbf{A} \cdot \mathbf{I}_{k}+\mathbf{F} .
$$


The matrix A has two important properties which are easily verified by direct computation:

$$
\mathbf{A}^{N}=\mathbf{1}, \quad \sum_{k=0}^{N-1} \mathbf{A}^{k}=0 .
$$

Applying equation (65) repeatedly and using these relations we get

$$
\mathbf{I}_{k+N}=\mathbf{A}^{N} \cdot \mathbf{I}_{k}+\sum_{k=0}^{N-1} \mathbf{A}^{k} \mathbf{F}=\mathbf{I}_{k}
$$

This shows explicitly that the cross-over currents are periodic in the longitudinal index $k$ with a period $N$, the number of strands in the cable. This conclusion rests on the assumption that the cross-over resistances and the magnetic flux are independent of the $z$ coordinate. In the previous section the eddy currents have been taken as independent of the longitudinal coordinate $z$. The present treatment shows that this is a special case which will be realized only if the appropriate initial conditions are satisfied. In general the cross-over currents will vary periodically with $z$ with a period $l_{p}$.

In addition to the cross-over resistances $R_{c}$ one can also take into account the resistances $R_{a}$ between adjacent strands which, however, are of minor importance concerning field distortions and a.c. losses. The most general case can only be studied in a computer simulation, see e.g. [55].

\subsection{Effect of cable eddy currents on field quality and magnet performance}

The small-loop cable eddy current create a bipolar current pattern as sketched in Fig. 32 and this will certainly generate multipole fields. In contrast to the filament magnetization currents, the eddy currents do not obey any coil symmetry because the cross-over resistances can vary from turn to turn and also in longitudinal direction. For this reason all allowed and unallowed multipoles are expected to appear during a ramp of the magnetic field, and their strength has to be proportional to the time derivative of $B$. Numerous measurements, especially from the former SSC laboratory, confirm this expectation. A good example are the strongly ramp-rate dependent quadrupole and sextupole fields in an SSC dipole (Fig. 34). The skew multipole fields $A_{2}, A_{3}, A_{5}, A_{5}$ are influenced in a similar manner. The time constants for the eddy-current multipoles are in the order of a second. It is interesting to note that the eddy-current induced sextupole is of opposite sign as the sextupole due to superconductor magnetization. The same has been observed in HERA dipoles. The apperance of 'forbidden' multipoles (normal quadrupole and skew multipoles) proves that the cable eddy currents do indeed violate the dipole coil symmetry, as stated above.

The multipoles can be correlated with measured a.c. losses and with a strong decrease of quench current at high ramp rates. In Fig. 35 the energy loss per current cycle $500 \mathrm{~A} \rightarrow 5000 \mathrm{~A} \rightarrow 500 \mathrm{~A}$ is plotted as a function of current ramp rate $d I / d t$. The reduction in quench current at a given ramp rate, say $d I / d t=90 \mathrm{~A} / \mathrm{s}$, is found to be correlated with the loss per cycle which proves that eddy-current heating of the coil is responsible for the premature quenches at higher ramp rates. The magnets with a particularly strong ramp rate sensitivity were characterized by unusually low contact resistances of about $2 \mu \Omega$.

All SSC magnets were made from cables with bare copper surface. It has been suspected that in problematic magnets like DCA312 the oxide layer formed during cable and coil production was too thin. Magnets with such an extreme ramp rate sensitivity are practically useless for an accelerator. Control of interstrand resistance is thus an important aspect of cable production. At ramp rate zero, the loss curves extrapolate to finite values which represent the hysteretic loss of the superconductor (compare Eq. (11)). For the current cycle chosen this loss is in the order of 600 to $800 \mathrm{~J}$ for the 15-m-long SSC magnets.

In Table 2 we reproduce part of a table from [53] showing predicted field distortions and power dissipation due to cable eddy currents in various dipole designs. The numbers refer to a uniform interstrand resistance $R_{c}=10 \mu \Omega$ and a ramp rate $d I / d t=10 \mathrm{~A} / \mathrm{s}$. The dipole, sextupole and decapole fields are given in $10^{-4}$ Tesla at a reference radius $r_{0}=25 \mathrm{~mm}$.

Loss measurements on a HERA dipole with ramp rates between 5 and $28 \mathrm{~A} / \mathrm{s}$ and a current cycle $1500 \mathrm{~A} \rightarrow 5500$ $\mathrm{A} \rightarrow 1500$ A revealed a linear relationship between energy loss $Q$ and ramp rate $d I / d t$ (H. Brück and M. Stolper, private communication):

$$
Q=Q_{h y s t}+Q^{\prime} \frac{d I}{d t}
$$

The constant term $Q_{\text {hyst }}=360 \mathrm{~J}$ represents the hysteretic loss, while the slope $Q^{\prime}=7.5 \mathrm{~J} /(\mathrm{A} / \mathrm{s})$ describes the eddy-current loss. The instantaneous power, dissipated by the eddy currents, is thus about $90 \mathrm{~mW}$ in the 9-m-long magnet for a ramp rate of $10 \mathrm{~A} / \mathrm{s}$. Using this value and Table 2 the average cross-over resistance is found to be $R_{c} \approx 10 \mu \Omega$. Then, for the standard HERA ramp rate of $10 \mathrm{~A} / \mathrm{s}$, the predicted field distortions caused by eddy currents should be in the order of $1.5 \cdot 10^{-4} \mathrm{~T}$ for the dipole and $0.4 \cdot 10^{-4} \mathrm{~T}$ for the sextupole term. Both numbers are in fairly good agreement with direct determinations of ramp-rate dependent multipoles. It is interesting to note that the hysteretic loss exceeds the eddy-current loss for the low ramp rates used in storage rings. 

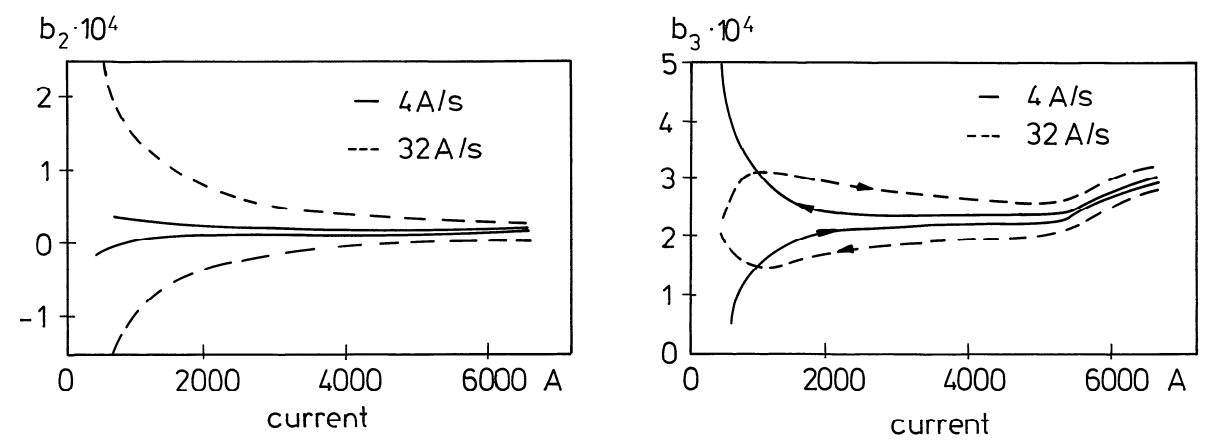

Figure 34: Hysteresis of the normal quadrupole and sextupole coefficients as a function of magnet current for ramp rates of 4 and $32 \mathrm{~A} / \mathrm{s}$. The ramp direction is indicated by arrows. The width of the hysteresis curves is proportional to $d I / d t$ which proves that eddy currents are the source. The measurements were performed at Brookhaven on dipole DCA312.
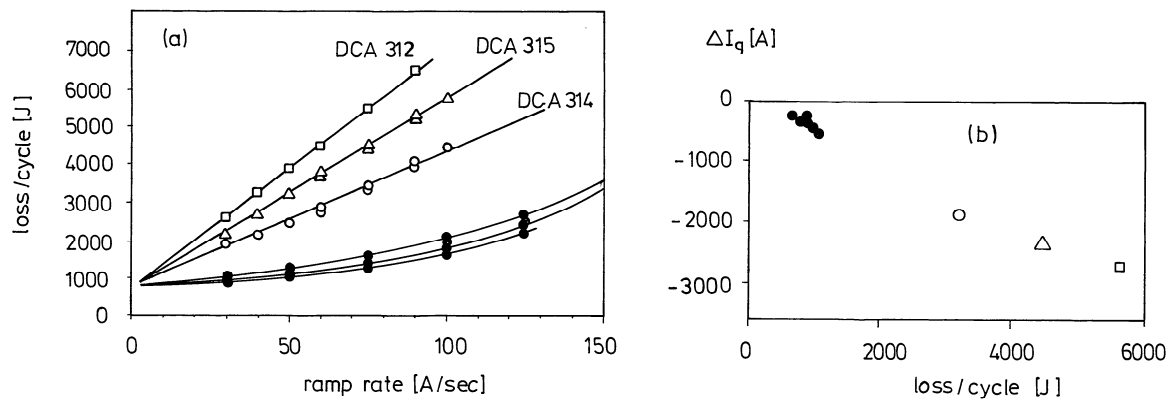

Figure 35: (a) Energy loss $Q$ per cycle as a function of current ramp rate $d I / d t$ for various SSC dipoles. Five dipoles with small losses are indicated by black dots. The three dipoles with particularly large losses are labelled by their serial numbers.

(b) Correlation between quench current reduction $\Delta I_{q}$ and energy loss $Q$ per cycle (both at $d I / d t=90 \mathrm{~A} / \mathrm{s}$ ) [56].

\subsection{Eddy currents in longitudinally vary- ing fields}

\subsubsection{Theoretical model}

It is suggestive to search for a relation between the periodic cross-over currents in the cable and the longitudinal periodicity observed in the persistent-current multipole fields. Krempaski and Schmidt [49] and independently Verweij and ten Kate [38] have investigated the possibility that the time derivative of the magnetic field varies along the cable direction. In accelerator magnets this is indeed realized since in the coil heads and at the current leads the magnetic field and hence $d B / d t$ differ from the values in the straight section. Also when going from one winding turn to another the local field and its time derivative will change.

Following the lucid treatment in [49] we consider a twostrand Rutherford cable whose length $l_{0}$ is much larger than the transposition pitch length $l_{p}$. We assume that $\dot{B}$ is nonzero in a limited length $b$ around the centre $z=l_{0} / 2$ and vanishes elsewhere. The arrangement is sketched in Fig. 36. The two wires are connected every half pitch by the contact resistance $R_{c}$. Since $l_{p} \ll l_{0}$ we may treat the two-wire system as a continuous transmission line and introduce a transverse conductance and

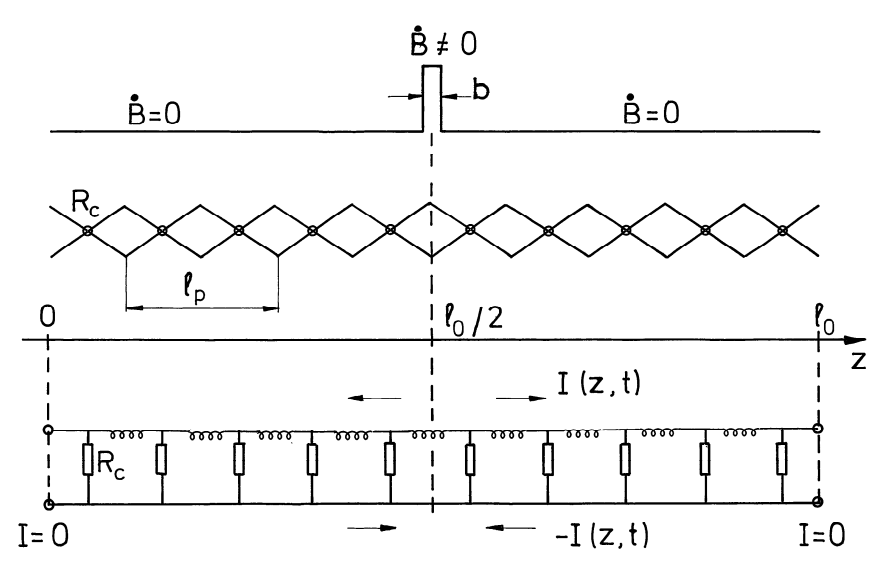

Figure 36: A two-strand cable subjected to a time-varying magnetic field in the centre region and the equivalent electric circuit.

an inductance per unit length:

$$
G^{\prime}=2 /\left(R_{c} l_{p}\right), \quad L^{\prime}=\left(\mu_{0} / \pi\right)\left(\ln \left(w / d_{s}\right)+0.25\right) .
$$

Here $w$ is the cable width and $d_{s}$ the strand diameter. The maximum induced voltage is obtained if the length $b$ is an odd multiple of half the transposition pitch length: $U=U_{\max }=l_{p} w \dot{B} / 4$ for $b=(2 k+1) l_{p} / 2$ while $U=0$ 
Table 2: Predicted field distortions and power dissipation due to cable eddy currents for various superconducting dipole magnet designs [53]. The numbers refer to a uniform cross-over resistance $R_{c}=10 \mu \Omega$ and a ramp rate $d I / d t=10$ A/s.

\begin{tabular}{|lccc|}
\hline & HERA & RHIC & Tevatron \\
\hline$B(I) / I \quad(\mathrm{~T} / \mathrm{kA})$ & 0.935 & 0.709 & 0.953 \\
& & & \\
Inner cable & & & \\
width $(\mathrm{mm})$ & 10 & 9.73 & 7.8 \\
twist pitch $(\mathrm{mm})$ & 95 & 74 & 57 \\
number of strands & 24 & 30 & 23 \\
& & & \\
Outer cable & & & \\
width $(\mathrm{mm})$ & 10 & - & 7.8 \\
twist pitch $(\mathrm{mm})$ & 95 & - & 57 \\
number of strands & 24 & - & 23 \\
& & & \\
Field distortions & & & 0.6 \\
$B_{1}\left(10^{-4} \mathrm{~T}\right)$ & 1.5 & 0.5 & 0.2 \\
$B_{3}\left(10^{-4} \mathrm{~T}\right)$ & +0.4 & +0.2 & -0.01 \\
$B_{5}\left(10^{-4} \mathrm{~T}\right)$ & -0.05 & -0.02 & \\
& & & \\
Power dissipation & & & \\
$\mathrm{P}\left(10^{-3} \mathrm{~W} / \mathrm{m}\right)$ & 9 & 3 &
\end{tabular}

for $b=k \cdot l_{p}$.

The currents $\pm I(z, t)$ in the wires must fulfill the differential equation of a transmission line.

$$
\frac{\partial^{2} I}{\partial z^{2}}=L^{\prime} C^{\prime} \frac{\partial^{2} I}{\partial t^{2}}+\left(R^{\prime} C^{\prime}+L^{\prime} G^{\prime}\right) \frac{\partial I}{\partial t}+R^{\prime} G^{\prime} I
$$

For vanishing longitudinal resistance and transverse capacitance $\left(R^{\prime}=0, C^{\prime}=0\right)$ the equation reduces to

$$
\frac{\partial^{2} I}{\partial z^{2}}=L^{\prime} G^{\prime} \frac{\partial I}{\partial t}
$$

This is a diffusion-like equation with diffusivity $D=$ $1 /\left(L^{\prime} G^{\prime}\right)$. In the steady state, when $t$ is large compared to the time constant of the system, the right-hand side of the equation is zero and the current is obviously a linear function of $z$ which vanishes at $z=0$ and $z=l_{0}$. The maximum $I_{\max }=U G^{\prime} l_{0} / 4$ occurs around $z=l_{0} / 2$. For a short extension of the magnetic field region, $b \ll l_{0}$, this is a triangle-like function ${ }^{8}$ which can be expanded in a Fourier series of period $2 l_{0}$.

$$
I(z)=\frac{8 I_{\max }}{\pi^{2}} \sum_{n=1,3,5 \ldots} \frac{(-1)^{(n-1) / 2}}{n^{2}} \sin \left(\frac{n \pi z}{l_{0}}\right) .
$$

\footnotetext{
${ }^{8}$ The more general case of an extended region with $\dot{B} \neq 0$ is treated in [49].
}

The solution $I(z, t)$ for the decay from the steady state is easy to construct. When the field increase is stopped at $t=0$, which means that $\dot{B}$ vanishes for $t>0$, one has to multiply the $n$th Fourier term with $\exp \left(-t / \tau_{n}\right)$. From Eq. (68) follows that the time constant $\tau_{n}$ of the Fourier term $n$ is given by

$$
\tau_{n}=\tau / n^{2} \quad \text { with } \quad \tau=\frac{L^{\prime} G^{\prime} l_{0}^{2}}{\pi^{2}} .
$$

The time constant $\tau=\tau_{1}$ can be quite large. If we consider a single winding turn in a 10-m-long dipole and assume a cross-over resistance $R_{c}=5 \mu \Omega$ and a transposition pitch length of $l_{p}=0.1 \mathrm{~m}$ then $\tau \approx 160 \mathrm{~s}$. The time constant grows with the square of the cable length. Moreover, in a multistrand Rutherford cable there are many more cross-over points than in our twostrand model, so the transverse conductance is much larger. As a consequence the time constant of a complete subcoil of the dipole may be in the order of many hours or even days.

The solution for the charging period is found in a similar way. If $\dot{B}$ is switched on at $t=0$ the current as a function of time is

$$
I(z, t)=\hat{I} \sum_{n=1,3,5 \ldots} \frac{(-1)^{(n-1) / 2}}{n^{2}} \sin \left(\frac{n \pi z}{l_{0}}\right)\left(1-\exp \left(-t / \tau_{n}\right)\right)
$$

with $\hat{I}=8 I_{\max } / \pi^{2}$. If charging is stopped at $t=t_{1}$ the components $I_{n}\left(z, t_{1}\right)$ of the sum in Eq. (70) decay with their respective time constants $\tau_{n}=\tau / n^{2}$ and the current for $t>t_{1}$ is

$$
I(z, t)=\sum_{n=1,3,5 \ldots} I_{n}\left(z, t_{1}\right) \exp \left(-\left(t-t_{1}\right) / \tau_{n}\right) .
$$

Of particular interest for the accelerator is a linear ramp cycle $0 \rightarrow B_{\max } \rightarrow 0$ as sketched in Fig. 37a. We assume a ramp-up time $t_{1}=\tau / 10$ and take the same interval for the dwell time at high field and the rampdown time. Using equations (68) to (71) the current $I(z, t)$ can be computed at all times. The parameters chosen are: cable length $l_{0}=20 \mathrm{~m}$, magnetic field region $b=l_{p} / 2=0.05 \mathrm{~m}$, contact resistance $R_{c}=5 \mu \Omega$, field ramp rate $\dot{B}=0.05 \mathrm{~T} / \mathrm{s}$. The results are plotted in Figs. $37 \mathrm{~b}, \mathrm{c}$. When the magnetic field has undergone the ramp cycle and is kept at $B=0$ afterwards $\left(t>t_{3}\right)$ there is still a considerable time dependence in the current. Eventually of course the current $I$ approaches zero but the decay time may be many hours for a complete magnet coil. It is obvious that the zig-zag current pattern generates a magnetic field with a longitudinal periodicity.

It should be noted that the induced currents are in the $50-100$ A range and thus not negligible in comparison with the typical transport current carried by a strand. This effect contributes to the ramp rate dependence of quench current, shown in Fig. 35, because the sum of transport and induced current may exceed the critical current of a strand. Eddy-current heating of the coil leads to an additional reduction of quench current. 

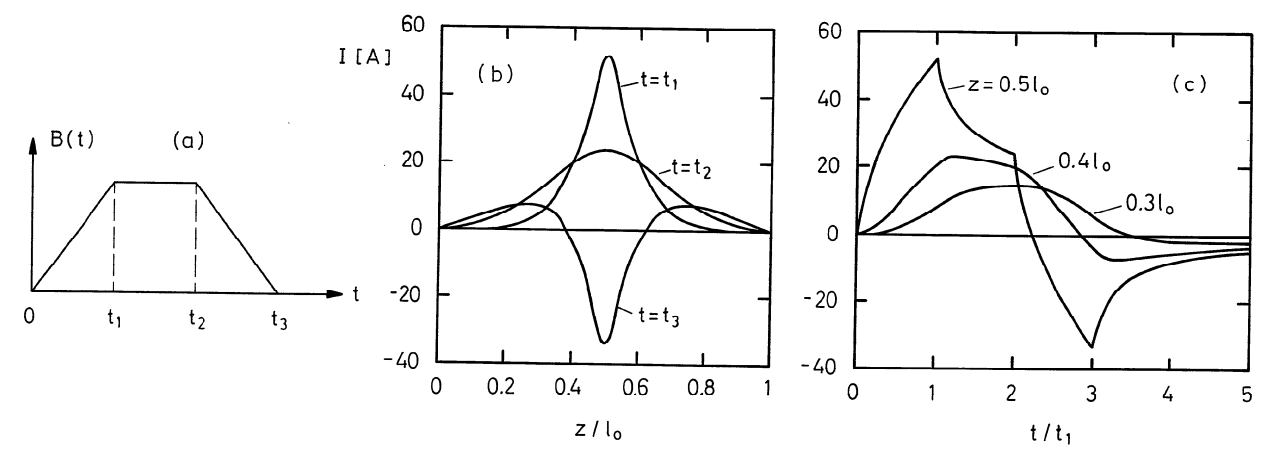

Figure 37: (a) Ramp cycle of magnetic field. (b) Position dependence of the current $I(z, t)$ for the times $t_{1}, t_{2}=2 t_{1}, t_{3}=3 t_{1}$. (c) Time dependence of $I(z, t)$ at various positions.

An experiment has been performed [38] to study the effect on a 1.3-m-long Rutherford cable. The cable was clamped with $15 \mathrm{MPa}$ over a length of $1.1 \mathrm{~m}$ to achieve a low cross-over resistance and was subjected to a timevarying magnetic field at one end. The magnetic field along the cable, measured with a Hall probe arrangement, exhibits indeed an oscillatory pattern with the cable transposition pitch as its period (see Fig. 38).

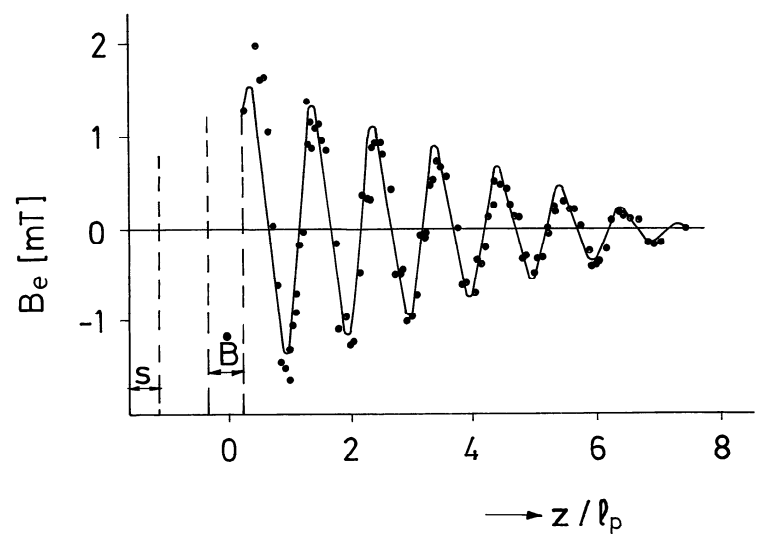

Figure 38: Experimental study of 'boundary-induced' eddy currents in a Rutherford cable [38]. The time-varying magnetic field $(\dot{B}=0.016 \mathrm{~T} / \mathrm{s})$ is concentrated around $z=0$. At the left end the cable has been soldered (labelled by $s$ in the drawing) to simulate the effect of an internal solder connection in a dipole coil. The eddy-current field $B_{e}$ along the cable is measured with an array of Hall probes. Continuous curve: model calculation.

\subsection{Influence on the persistent-current effects in magnets}

The induction effect caused by the variation of $\dot{B}$ along the cable of a coil can qualitatively account for the longitudinal periodicity seen in all magnets. The observed multipole field pattern results from a complex superposition of the bipolar currents induced in any pair of strands and in all turns of the coil. Since the cross-over resistances and the number of pitch lengths between the coil heads will vary from turn to turn and from magnet to magnet, rather different oscillation patterns of the various multipoles must be expected for different magnets. For this reason a quantitative analysis appears not very meaningful and predictions for a new magnet will be difficult.

Nevertheless, some important features can be understood in a qualitative way. We have seen in Fig. 30 that the oscillation amplitude is very small at the initial excitation of a magnet but grows considerably with increasing field. This is obvious from the factor $\left(1-\exp \left(-t / \tau_{n}\right)\right)$ in Eq. (70): if the ramp time is much shorter than the characteristic time constant $\tau$ or, alternatively, if $\dot{B}$ is very small, the induced current will be tiny. On the contrary, long ramps at sufficiently large $d B / d t$ will generate strong oscillations. Even if the field is reduced to zero afterwards or to a small value, the oscillations remain large as is evident from Fig. 37c.

These 'boundary-induced' eddy currents offer also a qualitative explanation why the decay rates of persistentcurrent multipoles depend so much on the pre-cycle (compare Fig. 29). If a large field sweep has been performed, significant eddy currents will be flowing in the strands whose decay times are in the order of many hours. The total current $I_{s}$ in a strand is the sum of the transport current $I_{t}$ and the respective eddy current $I_{e}$; it may decrease or increase in the course of time depending on the sign of $I_{e}$. What is the effect of this time dependence on the superconductor magnetization? We make the important observation that any change of the strand current, either positive or negative, is accompanied with a reduction of the overall strand magnetization. This can be understood as follows. A current change $+\Delta I_{s}$ creates an azimuthal magnetic field change $\Delta B_{\phi}$ inside the strand. From the large previous field sweep all filaments in the strand have been left fully magnetized. In one hemisphere of the strand, $\Delta B_{\phi}$ has a component parallel to the field that magnetized the strand. The filaments in this region keep their magnetization because they are al- 
ready saturated. In the other hemisphere, however, $\Delta B_{\phi}$ opposes the previous magnetizing field and here the filament magnetization is reduced.

For a negative current change $-\Delta I_{s}$ the two hemispheres are interchanged but the overall strand magnetization is reduced as well. Our conclusion is that the strong magnetization decay, observed after large field sweeps, is caused by a current redistribution among the strands in the cable. The above model provides the underlying physical mechanism. Again quantitative predictions appear difficult because of the many unknowns in the problem. The large magnet-to-magnet variation in the decay rates is most likely caused by large variations in cross-over resistances and different numbers of transposition pitch lengths between the coil ends.

\subsection{Transmission line characteristics of a long string of magnets}

\subsubsection{Equivalent circuit of a dipole}

A superconducting dipole cannot be considered as a pure inductance, in spite of the vanishing resistance of the conductor. We have seen above that eddy current losses play a significant role. In an equivalent circuit they correspond to a frequency-dependent real part of the impedance. Furthermore, the tight compression of the windings, provided by the collars, introduces a sizeable capacitance. Following R. Shafer [57] we consider as a simple model a dipole equipped with an electrically conducting beam pipe and neglect for the moment eddy currents in the cable or collars. Let $L_{1}$ be the d.c. inductance of the magnet coil (measured in the limit of vanishing frequency), $l_{1}$ its length and $a$ its average radius. The beam pipe is influenced by the time-varying field over the same length $l_{1}$. The inductance and resistance for induced eddy currents are

$$
L_{2}=\mu_{0} l_{1} /(4 \pi), \quad R_{2}=\frac{2 l_{1} \rho}{\pi b t} .
$$

Here $b$ is the pipe radius, $\rho$ its resistivity and $t$ the wall thickness. The mutual inductance of coil and pipe is given by the expression

$$
M=\frac{b}{a} \sqrt{L_{1} L_{2}}
$$

Connecting the coil to an a.c. voltage $U_{1} \exp i \omega t$ we obtain the equations

$U_{1}=i \omega L_{1} I_{1}+i \omega M I_{2} \quad 0=\left(R_{2}+i \omega L_{2}\right) I_{2}+i \omega M I_{1}$.

The impedance of the magnet coil with inserted beam pipe is

$$
Z_{\text {coil }}(\omega)=\frac{U_{1}}{I_{1}}=\left(i \omega L_{1}+\frac{\omega^{2} M^{2}}{R_{2}+i \omega L_{2}}\right)
$$

Separating real and imaginary parts this can be written as

$$
Z_{\text {coil }}(\omega)=\frac{\kappa L_{1} \tau \omega^{2}}{1+\omega^{2} \tau^{2}}+i \omega\left[(1-\kappa) L_{1}+\frac{\kappa L_{1}}{1+\omega^{2} \tau^{2}}\right] .
$$

Here $\kappa=b^{2} / a^{2}$ and $\tau=L_{2} / R_{2}=\mu_{0} b t /(2 \rho)$. The impedance of Eq. (72) corresponds to the circuit drawn in Fig. 39a.
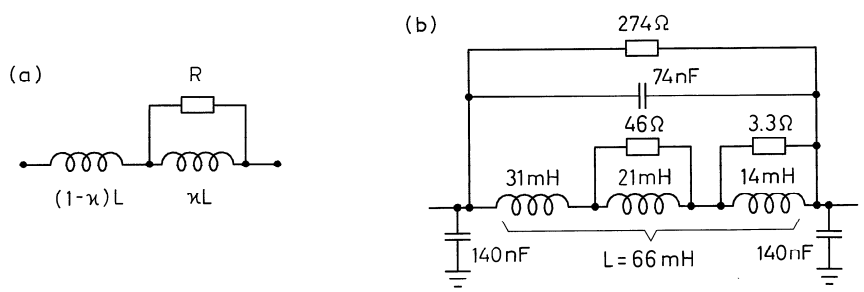

Figure 39: (a) Equivalent circuit for a dipole with inductively coupled conducting beam pipe. (b) Equivalent circuit of SSC dipole including cable capacitance [58].

The capacity between windings can be replaced by an equivalent capacitance against ground potential. If all eddy currents are taken into consideration one arrives at the equivalent circuit in Fig. 39b. The frequency response of a superconducting dipole is indeed that of a damped resonance circuit, see Fig. 40. The parameters
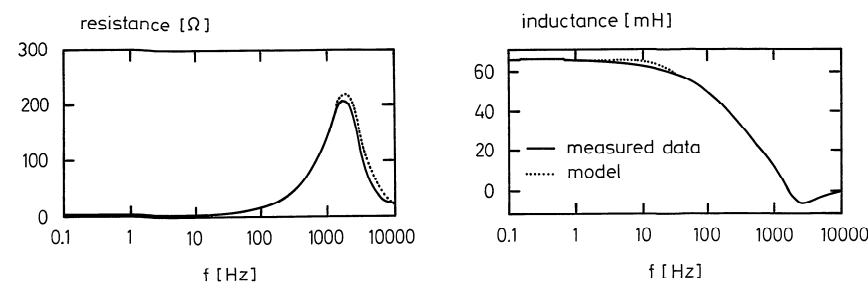

Figure 40: The measured resistance (real part of the impedance) and inductance (imaginary part divided by $\omega$ ) of an SSC dipole as a function of frequency. The prediction based on the equivalent circuit of Fig. 39b is shown for comparison [58].

of the equivalent circuit have been adjusted to yield the best agreement with the measurement.

\subsubsection{String of magnets}

A long string of dipoles can be considered as a transmission line. In the low-frequency limit, $\omega \ll 1 / \tau$, the string has a characteristic impedance and a phase velocity

$$
Z=\sqrt{\frac{L^{\prime}}{C^{\prime}}} \approx 900 \Omega, \quad v=\frac{1}{\sqrt{L^{\prime} C^{\prime}}}
$$

where $L^{\prime}$ and $C^{\prime}$ are the inductance and capacitance per unit length. The phase velocity is in the order of 

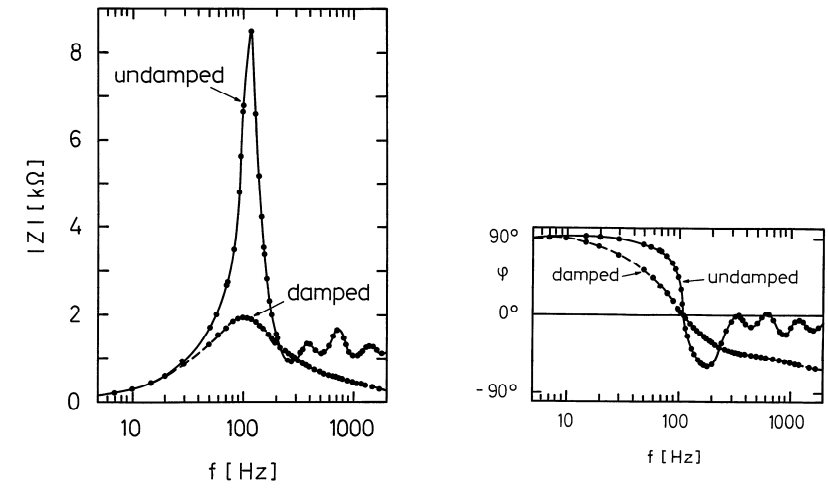

Figure 41: Observed resonance behaviour in a 96-magnet string in the Tevatron. Shown is the absolute value of the impedance and the phase as a function of frequency for the undamped and the damped case. Curves: model calculation. Dots: measured data [58]

$100 \mathrm{~km} / \mathrm{s}$ or about 10.000 to 20.000 magnets per second. At frequencies $\omega>1 / \tau$ transmission line effects become significant. Figure 41 shows data from a section of the Tevatron comprising 96 magnets. The absolute magnitude of the impedance and the phase are plotted as a function of frequency. A pronounced resonance is observed at $100 \mathrm{~Hz}$ and some higher resonances in addition. Measurement and model calculation agree very well. Such a resonance is very undesirable as it may lead to voltage enhancements during a magnetic field ramp or to a variation of the dipole field along the chain if power supply ripple excites the resonance frequency. Fortunately it can be strongly damped by an external resistors of some $100 \Omega$ which is connected parallel to the thyristor or diode used for quench protection (see sect. 4.5.5). The transmission-line properties of the superconducting magnet chain are a potential danger in the case of magnet quenches. Large voltage transients may propagate along the magnet chain leading to enhanced coil-to-ground voltages.

\section{Design Principles and Proper- ties of Superconducting Cavi- ties}

\subsection{Choice of superconductor}

In principle the critical temperature of the superconductor should be as high as possible. However, copper cavities coated with a high- $T_{c}$ superconductor layer have shown unsatisfactory performance [24], therefore the helium-cooled low- $T_{c}$ superconductors are applied. In contrast to magnets were hard superconductors with large upper critical field (10-20 T) are needed, the superconductor in microwave applications is not limited by the upper critical field but rather by the thermodynamic critical field (or possibly the 'superheating field') which is well below $0.5 \mathrm{~T}$ for all known superconducting elements and alloys. Moreover, strong flux pinning appears undesirable as it is coupled with hysteretic losses. Hence a 'soft' superconductor must be used, and pure niobium is the best candidate although its critical temperature is only $9.2 \mathrm{~K}$ and the thermodynamic critical field about $200 \mathrm{mT}$. Niobium-tin $\left(\mathrm{Nb}_{3} \mathrm{Sn}\right)$ looks more favorable at first sight since it has a higher critical temperature of $18 \mathrm{~K}$ and a superheating field of $400 \mathrm{mT}$; however, the gradients achieved in $\mathrm{Nb}_{3} \mathrm{Sn}$ coated copper cavities were below $15 \mathrm{MV} / \mathrm{m}$, probably due to grain boundary effects in the $\mathrm{Nb}_{3} \mathrm{Sn}$ layer [59]. For these reasons pure niobium has been chosen in all large scale installations of sc cavities. Here two alternatives exist: the cavities are fabricated from solid niobium sheets, or a thin niobium layer is sputtered onto the inner surface of a copper cavity. Both approaches have been successfully applied, the former one at Cornell (CESR), KEK (TRISTAN), DESY (PETRA, HERA, TESLA Test Facility TTF), Darmstadt (S-DALINAC), Jefferson Lab (CEBAF) and other laboratories, the latter one in particular at CERN in the electron-positron storage ring $\mathrm{LEP}^{9}$.

The microwave surface resistance is according to the Bardeen Cooper Schrieffer theory

$$
R_{B C S} \propto \lambda_{L}^{3} \omega^{2} \ell \exp \left(-1.76 T_{c} / T\right) .
$$

This formula applies if the mean free path $\ell$ of the unpaired electrons is much larger than the coherence length $\xi$. In niobium this condition is usually not fulfilled and one has to replace $\lambda_{L}$ in the above equation by [60]

$$
\Lambda=\lambda_{L} \sqrt{1+\xi / \ell} .
$$

Combining equations (73) and (74) we arrive at the surprising statement that the surface resistance does not assume its minimum value when the superconductor is of very high purity $(\ell \gg \xi)$ but rather in the range $\ell \approx \xi$. Experimental results [61] and theoretical models [62] confirm this prediction. The effect is also observed in copper cavities with a thin niobium sputter coating in which the electron mean free path is in the order of $\xi$. At $4.2 \mathrm{~K}$ the quality factors in the LEP cavities are indeed a factor of two higher than in pure niobium cavities [63].

In addition to the BCS term there is a residual resistance caused by impurities, frozen-in magnetic flux or lattice distortions.

$$
R_{\text {surf }}=R_{B C S}+R_{\text {res }} .
$$

$R_{r e s}$ is temperature independent and amounts to a few $\mathrm{n} \Omega$ for a clean niobium surface but may readily increase if the surface is contaminated.

\footnotetext{
${ }^{9}$ The parameters of the colliders CESR, TRISTAN, PETRA, HERA, LEP are summarized in [1]. The S-DALINAC and the Continuous Electron Beam Accelerator Facility CEBAF [11] are recirculating superconducting linacs for nuclear physics and free electron laser application.
} 
For niobium the BCS surface resistance at $1.3 \mathrm{GHz}$ amounts to about $800 \mathrm{n} \Omega$ at $4.2 \mathrm{~K}$ and drops to $15 \mathrm{n} \Omega$ at $2 \mathrm{~K}$, see Fig. 42. The exponential temperature dependence is the reason why operation at $2 \mathrm{~K}$ is essential for achieving high accelerating gradients in combination with very high quality factors. Superfluid helium is an excellent coolant owing to its high heat conductivity.

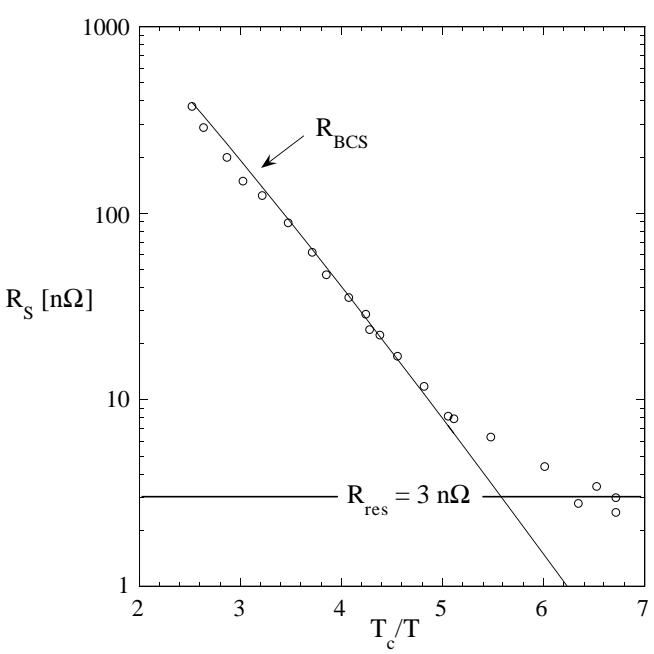

Figure 42: The surface resistance of a 9-cell TESLA cavity plotted as a function of $T_{c} / T$. The residual resistance of $3 n \Omega$ corresponds to a quality factor $Q_{0}=10^{11}$.

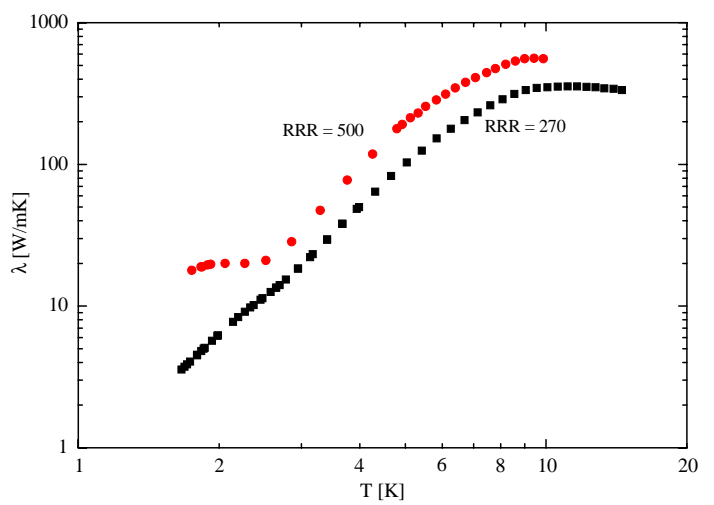

Figure 43: Measured heat conductivity in niobium samples with $R R R=270$ and $R R R=500$ as a function of temperature [65].

\subsection{Heat conduction in niobium and heat transfer to the coolant}

The heat produced at the inner cavity surface has to be guided through the cavity wall to the superfluid helium bath. At 2 - $4 \mathrm{~K}$, impurities have a strong impact on the thermal conductivity of metals. Niobium of very high purity is needed (contamination in the ppm range). The heat conductivity drops by about an order of magnitude when lowering the temperature from 4 to $2 \mathrm{~K}$, as shown in Fig. 43. The residual resistivity ratio $R R R=R(300 K) / R(10 K)$ is a good measure for the purity of the material: large $R R R$ means high electrical and thermal conductivity at low temperature.

Low frequency cavities (350-500 MHz) have a small BCS surface resistance at $4.2 \mathrm{~K}$ and are effectively cooled by normal liquid helium. The heat flux should not exceed a few $\mathrm{kW} / \mathrm{m}^{2}$ to obtain nucleate boiling with a close contact between liquid and metal. At higher heat fluxes one enters the film boiling regime where a vapour film covers the surface. Here the cavity may easily warm up beyond $T_{c}$ at areas of excessive heating. The $f^{2}$ dependence of the BCS resistance implies that for cavities of higher frequency superfluid helium at $1.8-2 \mathrm{~K}$ is more appropriate. At the metal-helium interface a temperature jump is observed which is attributed to phonon mismatch. The so-called Kapitza resistance amounts to about $1.5 \cdot 10^{-4} \mathrm{~m}^{2} \mathrm{~K} / \mathrm{W}$ [64] for a clean niobium surface in contact with superfluid helium.

\subsection{Influence of magnetic fields}

\subsubsection{Limitation of gradient}

Superconductivity breaks down when the microwave magnetic field at the cavity surface exceeds a critical value which is close to the thermodynamic critical field $B_{c}$ (200 mT for niobium at 2 Kelvin). The corresponding accelerating field on the cavity axis is about $50 \mathrm{MV} / \mathrm{m}$. It has been claimed that rf superconductivity persists up to a so-called 'superheating field', exceeding $B_{c}$ by $20 \%$ in the case of $\mathrm{Nb}$ [23], [66]. However it remains to be proven that rf cavities cooled by superfluid helium can be reliably operated near or even beyond $B_{c}$.

\subsubsection{Trapped magnetic flux}

Niobium is a soft type II superconductor without strong flux pinning, however, weak magnetic dc fields are not expelled upon cooldown but remain trapped in the niobium. Each flux line contains a normal-conducting core. Trapped magnetic dc flux therefore results in a surface resistance [60]

$$
R_{m a g}=\frac{B_{e x t}}{2 B_{c 2}} R_{n}
$$

where $B_{e x t}$ is the externally applied field, $B_{c 2}$ the upper critical field and $R_{n}$ the surface resistance in the normal state. At $1.3 \mathrm{GHz}$ the surface resistance caused by trapped flux amounts to $3.5 \mathrm{n} \Omega / \mu \mathrm{T}$ for niobium. Cavities from solid niobium which are not shielded from the Earth's magnetic field are limited to $Q_{0}$ values below $10^{9}$. However, copper cavities with a thin niobium sputter coating are found to be quite insensitive to magnetic fields and must not be shielded. The reason is that the sputter layer has a larger $B_{c 2}$ than bulk niobium because of impurities and defects. 


\subsection{Pill box cavity}

The simplest model of an accelerating cavity is a hollow cylinder which is often called pill box. When the beam pipes are neglected the field pattern inside the resonator and all relevant cavity parameters can be calculated analytically.

\subsubsection{Field pattern}

For particle acceleration we need a longitudinal electric field on the axis, hence we look for TM (transverse magnetic) eigenmodes of the cylindrical resonator. The field lines are sketched in Fig. 44. We use cylindrical co-

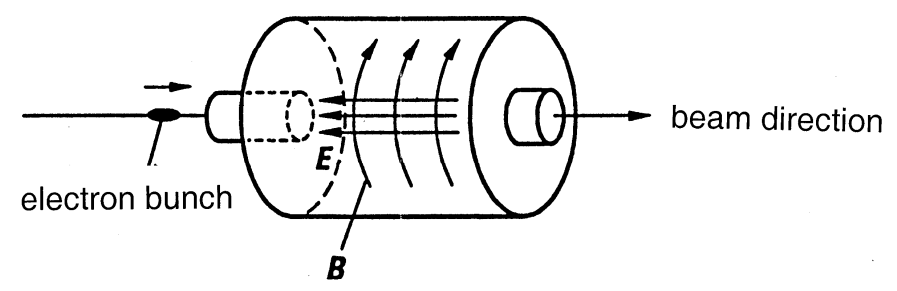

Figure 44: Electric and magnetic field in a pillbox cavity for the accelerating mode $T M_{010}$.

ordinates $(r, \theta, z)$ where $z$ denotes the beam direction (cavity axis), $r=\sqrt{x^{2}+y^{2}}$ and $\theta$ the azimuthal angle. We search for an eigenmode with cylindrical symmetry (independence of $\theta$ ) and with longitudinal electric and azimuthal magnetic field. The wave equation for the electric field reads

$$
\frac{\partial^{2} E_{z}}{\partial r^{2}}+\frac{1}{r} \frac{\partial E_{z}}{\partial r}=\frac{1}{c^{2}} \frac{\partial^{2} E_{z}}{\partial t^{2}} .
$$

For a harmonic time dependence $E_{z}(r) \cos (\omega t)$ and with the new variable $u=r \omega / c$ one obtains

$$
\frac{\partial^{2} E_{z}}{\partial u^{2}}+\frac{1}{u} \frac{\partial E_{z}}{\partial u}+E_{z}(u)=0
$$

This is the Bessel equation of zero order with the solution $J_{0}(u)$. Hence the radial dependence of the electric field is

$$
E_{z}(r)=E_{0} J_{0}\left(\frac{\omega r}{c}\right)
$$

For a perfectly conducting cylinder of radius $R$ the longitudinal electric field must vanish at $r=R$, so $J_{0}(\omega R / c)=0$. The first zero of $J_{0}(u)$ is at $u=2.405$. This defines the frequency of the lowest eigenmode (we call it the fundamental mode in the following):

$$
f_{0}=\frac{2.405 c}{2 \pi R}, \quad \omega_{0}=\frac{2.405 c}{R} .
$$

In a cylindrical cavity the frequency does not depend on the length $L_{c}$. The magnetic field can be computed from the equation

$$
\frac{\partial E_{z}}{\partial r}=\mu_{0} \frac{\partial H_{\theta}}{\partial t}
$$

Hence we obtain for the fundamental TM mode

$$
\begin{aligned}
& E_{z}(r, t)=E_{0} J_{0}\left(\frac{\omega_{0} r}{c}\right) \cos \left(\omega_{0} t\right), \\
& H_{\theta}(r, t)=-\frac{E_{0}}{\mu_{0} c} J_{1}\left(\frac{\omega_{0} r}{c}\right) \sin \left(\omega_{0} t\right) .
\end{aligned}
$$

Electric and magnetic field are $90^{\circ}$ out of phase. The azimuthal magnetic field vanishes on the axis and assumes its maximum close to the cavity wall.

\subsubsection{Stored energy}

The electromagnetic field energy is computed by integrating the energy density $\left(\varepsilon_{0} / 2\right) E^{2}$ (at time $t=0$ ) over the volume of the cavity. This yields

$$
\begin{aligned}
U & =\frac{\varepsilon_{0}}{2} 2 \pi L_{c} E_{0}^{2} \int_{0}^{R} J_{0}^{2}\left(\frac{\omega_{0} r}{c}\right) r d r \\
& =\frac{\varepsilon_{0}}{2} 2 \pi L_{c} E_{0}^{2}\left(\frac{c}{\omega_{0}}\right)^{2} \int_{0}^{a} J_{0}^{2}(u) u d u
\end{aligned}
$$

where $a=2.405$ is the first zero of $J_{0}$. Using the relation $\int_{0}^{a} J_{0}^{2}(u) u d u=0.5\left(a J_{1}(a)\right)^{2}$ we get for the energy stored in the cavity

$$
U=\frac{\varepsilon_{0}}{2} E_{0}^{2}\left(J_{1}(2.405)\right)^{2} \pi R^{2} L_{c} .
$$

\subsubsection{Power dissipation in the cavity}

We consider first a cavity made from copper. The rf electric field causes basically no losses since its tangential component vanishes at the cavity wall while the azimuthal magnetic field penetrates into the wall with exponential attenuation and induces currents within the skin depth ${ }^{10}$. These alternating currents give rise to Ohmic heat generation. The skin depth is given by

$$
\delta=\sqrt{\frac{2}{\mu_{0} \omega \sigma}}
$$

where $\sigma$ is the conductivity of the metal. For copper at room temperature and a frequency of $1 \mathrm{GHz}$ the skin depth is $\delta=2 \mu \mathrm{m}$. Consider now a small surface element. From Ampere's law $\oint \vec{H} \cdot \overrightarrow{d s}=I$ follows that the current density in the skin depth is related to the azimuthal magnetic field by $j=H_{\theta} / \delta$. Then the dissipated power per unit area is ${ }^{11}$

$$
\frac{d P_{\text {diss }}}{d A}=\frac{1}{2 \sigma \delta} H_{\theta}^{2}=\frac{1}{2} R_{\text {surf }} H_{\theta}^{2} .
$$

Here we have introduced a very important quantity for rf cavities, the surface resistance:

$$
R_{\text {surf }}=\frac{1}{\sigma \delta} .
$$

\footnotetext{
${ }^{10}$ For a very careful discussion of the skin effect see J.D. Jackson, Classical Electrodynamics, chapt. 8.

${ }^{11}$ In equation (86) the quantity $H_{\theta}$ denotes the amplitude of the magnetic field without the periodic time factor $\sin \left(\omega_{0} t\right)$.
} 
In a superconducting cavity $R_{\text {surf }}$ is given by equations (73) to (75). The power density has to be integrated over the whole inner surface of the cavity. This is straightforward for the cylindrical mantle where $H_{\theta}=\frac{E_{0}}{\mu_{0} c} J_{1}\left(\omega_{0} R / c\right)$ is constant. To compute the power dissipation in the circular end plates one has to evaluate the integral $\int_{0}^{a}\left(J_{1}(u)\right)^{2} u d u=a^{2}\left(J_{1}(a)\right)^{2} / 2$. Again $a=2.405$ is the first zero of $J_{0}$. The total dissipated power in the cavity walls is then

$$
P_{\text {diss }}=R_{\text {surf }} \cdot \frac{E_{0}^{2}}{2 \mu_{0}^{2} c^{2}}\left(J_{1}(2.405)\right)^{2} 2 \pi R L_{c}\left(1+R / L_{c}\right) \text {. }
$$

\subsubsection{Quality factor}

The quality factor is an important parameter of a resonating cavity. It is defined as $2 \pi$ times the number of cycles needed to dissipate the stored energy, or, alternatively, as the ratio of resonance frequency $f_{0}$ to the full width at half height $\Delta f$ of the resonance curve

$$
Q_{0}=2 \pi \cdot \frac{U f_{0}}{P_{\text {diss }}}=\frac{f_{0}}{\Delta f} .
$$

Using the formulas (84) and (88) we get the important equation

$$
Q_{0}=\frac{G}{R_{\text {surf }}} \quad \text { with } \quad G=\frac{2.405 \mu_{0} c}{2\left(1+R / L_{c}\right)}
$$

which states that the quality factor of a cavity is obtained by dividing the so-called 'geometry constant' $G$ by the surface resistance. $G$ depends only on the shape of the cavity and not on the material. A typical value is 300 $\Omega$. We want to point out that the quality factor $Q_{0}$ defined here is the intrinsic or 'unloaded' quality factor of a cavity. If the cavity is connected to an external load resistor by means of a coupler another quality factor $\left(Q_{\text {ext }}\right)$ has to be introduced to account for the energy extraction through the coupler (see Sect. 8).

\subsubsection{Accelerating field, peak electric and mag- netic fields}

A relativistic particle needs a time $L_{c} / c$ to travel through the cavity. During this time the longitudinal electric field changes. The accelerating field is defined as the average field seen by the particle

$$
E_{a c c}=\frac{1}{L_{c}} \int_{-L_{c} / 2}^{L_{c} / 2} E_{0} \cos \left(\omega_{0} z / c\right) d z, \quad V_{a c c}=E_{a c c} L_{c}
$$

Choosing a cell length of one half the rf wavelength, $L_{c}=$ $c /\left(2 f_{0}\right)$, we get $E_{a c c}=0.64 E_{0}$ for a pill box cavity.

The peak electric field at the cavity wall is $E_{0}$. The peak magnetic follows from eq. (82). We get

$$
E_{\text {peak }} / E_{\text {acc }}=1.57, B_{\text {peak }} / E_{\text {acc }}=2.7 \mathrm{mT} /(\mathrm{MV} / \mathrm{m}) \text {. }
$$

If one adds beam pipes to the cavity these number increase by $20-30 \%$.

\subsection{Cavity shape}

The first sc cavities were built in the late 1960's with the conventional pill-box shape. They showed unexpected performance limitations: at fields levels of a few $\mathrm{MV} / \mathrm{m}$ a phenomen called multiple impacting (or multipacting for short) was observed. The effect is as follows: stray electrons which are emitted from the wall (for instance by cosmic rays) gain energy in one half-period of the electromagnetic field and return to their origin in the next half period were they impinge with a few $100 \mathrm{eV}$ onto the wall and release secondary electrons which repeat the same procedure. This way an avalanche of electrons is created which absorbs energy from the rf field, heats the superconductor and eventually leads to a breakdown of superconductivity. It was found out many years later that this problem is avoided in cavities having the shape of a rotational ellipsoid. When electrons are emitted near the iris of an elliptical cavity and accelerated by the rf field, they return to a point away from their origin in the next half period, and the same applies for the possible next generations of electrons. Thereby the daughter electrons move more and more into the equator region where the rf electric field is small and the multiplication process dies out. For a thorough discussion I refer to $[23]$.

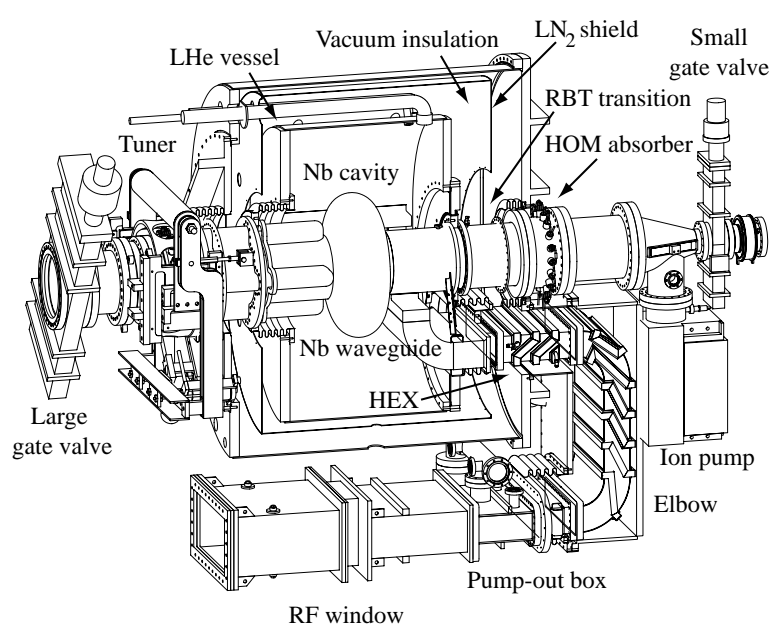

Figure 45: A $500 \mathrm{MHz}$ 1-cell niobium cavity used in the CESR storage ring at Cornell University. The cavity is equipped with a wave guide power coupler and cooled by normal liquid helium.

In electron-positron storage rings quite often singlecell cavities are used. These are particularly well suited for the large beam currents of up to $1 \mathrm{~A}$ in the high luminosity 'B meson factories'. Figure 45 shows a $500 \mathrm{MHz}$ cavity of the CESR machine which is made from solid niobium and equipped with a waveguide input coupler. At larger energies like in LEP (104 GeV per beam) multicell cavities are more efficient to compensate for the huge synchrotron radiation losses $(3 \mathrm{GeV}$ per revolution 
in LEP). In a linear collider almost the full length of the machine must be filled with accelerating structures and then long multicell cavities are mandatory. There are, however, several effects which limit the number of cells $N_{c}$ per resonator. With increasing $N_{c}$ it becomes more and more difficult to tune the resonator for equal field amplitude in every cell. Secondly, in a very long multicell cavity 'trapped modes' may be excited by the short particle bunches. These are coupled oscillations at high frequency which are confined to the inner cells and have such a low amplitude in the beam pipe sections that they cannot be extracted by a higher-order mode coupler. Trapped modes have a negative influence on the following bunches and must be avoided. The number $N_{c}=9$ chosen for TESLA appears a reasonable upper limit. The TESLA cavity [67] is shown in Fig. 46.
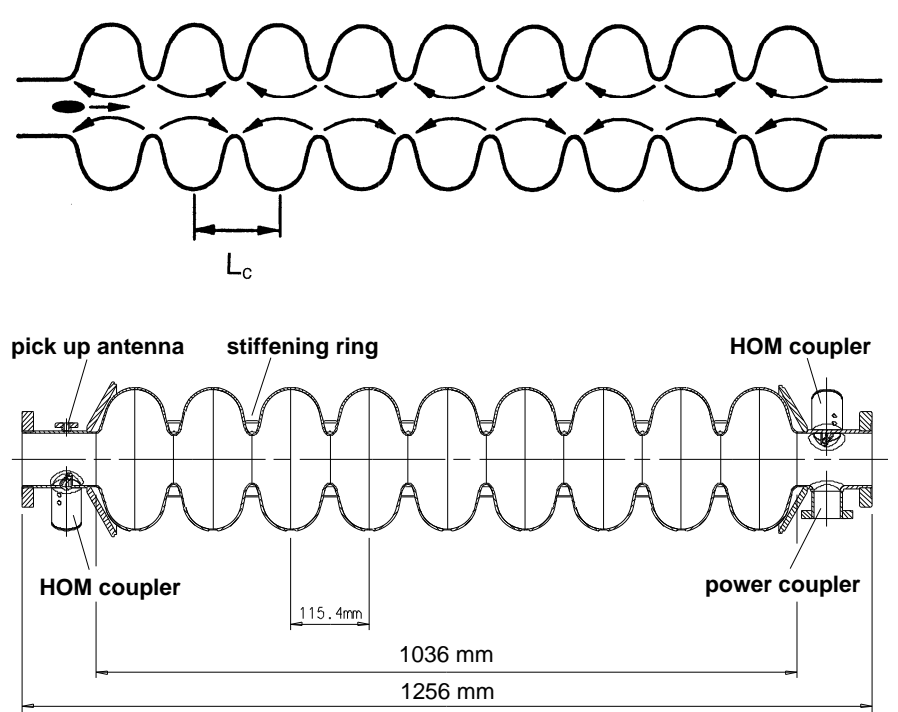

Figure 46: Top: Schematic cross section of the $1 \mathrm{~m}$ long 9-cell TESLA cavity with electric field lines. The resonance frequency is $1.3 \mathrm{GHz}$ and the cavity is operated in the $\pi$ mode with $180^{\circ}$ phase advance of the rf wave from cell to cell. The cell length equals $1 / 2$ the $r f$ wavelength so that relativistic electrons recieve the same energy gain in each cell. Bottom: Technical layout of the TESLA cavity with stiffening rings between neighbouring cells, two higher-order mode (HOM) couplers and flanges for mounting the rf power coupler and the pick-up antenna.

Superconducting cavities are always operated in standing-wave mode ${ }^{12}$. The fundamental $\mathrm{TM}_{010}$ mode

\footnotetext{
${ }^{12}$ In normal-conducting linacs like SLAC the travelling wave mode may be chosen. Basically the electrons 'ride' on the crests of the rf wave which propagates with the speed of light. In a superconducting linac a travelling wave is not attenuated by wall losses, and in order to preserve the basic advantage of superconductivity - almost no rf power is wasted - one would have to extract the rf wave after some length and feed it back through a superconducting wave guide to the input coupler. The required precision in rf phase would be extremely demanding and would make such a system far more complicated than a standing-wave linac.
}

is chosen with longitudinal electric field on the axis. In a cavity with $N_{c}$ cells the fundamental mode splits into $N_{c}$ coupled modes. The $\pi$ mode with $180^{\circ}$ phase difference between adjacent cells transfers the highest possible energy to the particles. The cell length $L_{c}$ is determined by the condition that the electric field has to be inverted in the time a relativistic particle needs to travel from one cell to the next, so $L_{c}=c /\left(2 f_{0}\right)$. For nonrelativistic protons or ions the cell length is $L_{c}=v /\left(2 f_{0}\right)$. The iris radius influences the cell-to-cell coupling parameter $k_{\text {cell }}$ which is in the order of $1-2 \%$. The frequencies of the coupled modes are given by the formula

$$
f_{m}=\frac{f_{0}}{\sqrt{1+2 k_{\text {cell }} \cos \left(m \pi / N_{c}\right)}}, \quad 1 \leq m \leq N_{c} .
$$

\subsection{Choice of frequency}

The losses in a microwave cavity are proportional to the product of conductor area and surface resistance. For a given length of a multicell resonator, the area scales with $1 / f$ while the surface resistance scales with $f^{2}$ for $R_{B C S} \gg R_{\text {res }}$ (see eq. (73)) and becomes independent of $f$ for $R_{B C S} \ll R_{\text {res }}$. At $T=2 \mathrm{~K}$ the BCS term dominates above $3 \mathrm{GHz}$ and here the losses grow linearly with frequency, whereas below $300 \mathrm{MHz}$ the residual resistance dominates and the losses are proportional to $1 / f$. To minimize power dissipation in the cavity wall one should therefore select $f$ in the range $300 \mathrm{MHz}$ to $3 \mathrm{GHz}$. Cavities in the 350 to $500 \mathrm{MHz}$ regime are commonly used in electron-positron storage rings. Their large size is advantageous to suppress wake field effects and losses from higher order modes. However, for a linac of several $10 \mathrm{~km}$ length the niobium and cryostat costs would be prohibitive for these bulky cavities, hence a higher frequency has to be chosen. Considering material costs $f=3 \mathrm{GHz}$ might appear the optimum but there are compelling arguments for choosing about half this frequency.

- The wake fields generated by the short electron bunches depend on radius as $1 / r^{2}$ for longitudinal and as $1 / r^{3}$ for transverse wakes. Since the iris radius of a cavity is inversely proportional to its eigenfrequency, the wake field losses scale with the second resp. third power of the frequency. Beam emittance growth and beam-induced cryogenic losses are therefore much higher at $3 \mathrm{GHz}$.

- The $f^{2}$ dependence of the BCS resistance makes a $3 \mathrm{GHz}$ cavity thermally unstable at gradients above $30 \mathrm{MV} / \mathrm{m}^{13}$, hence choosing this frequency would preclude a possible upgrade of the TESLA collider to $35 \mathrm{MV} / \mathrm{m}$ [68].

\footnotetext{
${ }^{13}$ See Fig. 11.22 in [23].
} 


\subsection{Technical limitations of sc cavities}

The fundamental advantage of superconducting cavities is their extremely low surface resistance of about $10 \mathrm{n} \Omega$ at $2 \mathrm{~K}$ leading to $\mathrm{rf}$ losses which are 5 to 6 orders of magnitude lower than in copper cavities. The drawback is that even tiny surface contaminations are potentially harmful as they decrease the quality factor and may even lead to a thermal breakdown (quench) of the superconductor due to local overheating.

\subsubsection{Thermal instability and field emission}

Field emission of electrons from sharp tips has been a notorious limitation of high-gradient sc cavities. The typical indication is that the quality factor drops exponentially above a certain threshold field, and $\mathrm{X}$ rays are observed. Temperature mapping at the outer cavity wall usually reveals that the heating by rf losses is not uniform over the whole surface but that certain spots exhibit larger temperature rises, often beyond the critical temperature of the superconductor. Hence the cavity becomes partially normal-conducting, associated with strongly enhanced power dissipation. Because of the exponential increase of surface resistance with temperature this may result in a run-away effect and eventually a quench of the entire cavity. Analytical models and numerical codes are available to describe this effect. The tolerable defect size depends on the purity of the material. As a typical number, the diameter of a normalconducting spot must be less than $50 \mu \mathrm{m}$ to avoid a thermal instability at $25 \mathrm{MV} / \mathrm{m}$.

The field emission current density is given by the Fowler-Nordheim equation [69], adapted for rf fields:

$$
j_{F E} \propto \frac{E_{l o c}^{2.5}}{\Phi} \exp \left(-C \Phi^{3 / 2} / E_{l o c}\right) \text {. }
$$

Here $\Phi$ is the work function of the metal, $C$ a constant and $E_{l o c}$ the local electric field which may be several 100 times larger than the accelerating field at sharp tips on the surface. The exponential behaviour can be observed by plotting the temperature rise at a 'hot spot' as a function of the reciprocal accelerating field. Perfect cleaning by rinsing with high-pressure ultrapure water is the most effective remedy against field emission. Using the clean room techniques developed in semiconductor industry it has been possible to raise the threshold for field emission in multicell cavities from about $10 \mathrm{MV} / \mathrm{m}$ to more than $20 \mathrm{MV} / \mathrm{m}$ in the past few years. Thermal instabilities and field emission are discussed at much greater detail in $[23]$.

\subsubsection{Lorentz-force detuning and microphonics}

The electromagnetic field exerts Lorentz forces on the currents which are induced in a thin surface layer. The resulting pressure acting on the cavity wall leads to a deformation of the cells in the $\mu \mathrm{m}$ range and a shift in resonance frequency which grows quadratically with the accelerating field. For a nine-cell $1.3 \mathrm{GHz}$ cavity with $2.5 \mathrm{~mm}$ wall thickness the frequency shift at $25 \mathrm{MV} / \mathrm{m}$ amounts to about $800 \mathrm{~Hz}$ and exceeds the width of the resonance curve of the cavity equipped with the main power coupler (see sect. 8.3). To reduce the detuning by a factor of two the TESLA cavities are mechanically reinforced by stiffening rings joining adjacent cells, see Fig. 46.

When the rf power is not applied continuously but in pulsed mode there is a time dependent detuning of the cavity. A measurement [70] is shown in Fig. 47. By using a piezo-electric actuator to adjust the cavity length appropriately the cavity eigenfrequency can be kept constant during the time interval used for beam acceleration

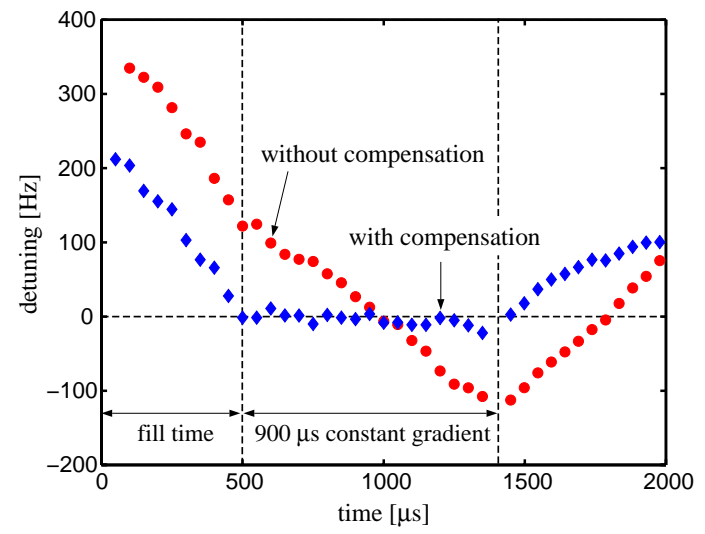

Figure 47: Frequency detuning of a TESLA cavity in pulsed operation at $23.5 \mathrm{MV} / \mathrm{m}$. Also shown is the frequency stabilization by means of a piezo-controlled tuner.

Mechanical vibrations of the cavity (so-called microphonics) result in random fluctuations of the cavity eigenfrequency. The rms frequency spread measured at the TESLA Test Facility is in the order of $10 \mathrm{~Hz}$ and well below the cavity bandwidth of $\approx 500 \mathrm{~Hz}$. It should be noted that microphonics becomes a critical issue in the multicell cavities foreseen for proton or ion acceleration at $\beta=v / c=0.5-0.8$ since the cells are flatter than in $\beta=1$ cavities and vulnerable to longitudinal oscillations.

\section{Performance of Practical Cavi- ties}

\subsection{Cavity fabrication}

As a typical example, I describe the fabrication of the TESLA cavities. Similar procedures were previously applied for the 340 five-cell resonators of the Continuous Electron Beam Accelerator Facility CEBAF [11]. In the first step, half-cells are produced from $2.8 \mathrm{~mm}$ thick niobium discs by deep-drawing. The half-cells are machined 
at the iris, cleaned by ultrasonic degreasing, $20 \mu \mathrm{m}$ chemical etching and clean water rinsing. Two half-cells are then joined at the iris by electron-beam (EB) welding to form a dumb-bell structure. The welding at the iris is usually done from the inside to ensure a smooth weld seam at the location of the highest electric field in the resonator. Since niobium is a strong getter material for oxygen it is important to carry out the EB welds in a vacuum of better than $5 \cdot 10^{-5}$ mbar. The next step is the welding of a stiffening ring. Frequency measurements are made on the dumb-bells to determine the correct amount of machining at the equators. After proper cleaning (a few $\mu \mathrm{m}$ etching followed by clean water rinsing and clean room drying), eight dumb-bells and two end half-cells with attached beam-pipe sections are stacked in a precise fixture. The EB welds at the equators are made from the outside. A reliable method for obtaining a smooth weld seam of a few mm width at the inner surface is to raster a slightly defocused electron beam in an elliptic pattern and to apply $50 \%$ of beam power during the first weld pass and $100 \%$ of beam power in the second pass.

\subsection{Cavity treatment}

Experience has shown that a damage layer in the order of $100 \mu \mathrm{m}$ has to be removed from the inner cavity surface to obtain good rf performance in the superconducting state. The standard method is called Buffered Chemical Polishing (BCP), using an acid mixture of $\mathrm{HF}$ (48\%), $\mathrm{HNO}_{3}(65 \%)$ and $\mathrm{H}_{3} \mathrm{PO}_{4}(85 \%)$ in the ratio 1:1:2 or 1:1:1. Afterwards the cavities are rinsed with ultra-clean water and then annealed at $800^{\circ} \mathrm{C}$ in an Ultra High Vacuum (UHV) oven to remove dissolved hydrogen from the niobium and to relieve mechanical stress. Many TESLA cavities have been tested after this step but the majority is subjected to a $1400^{\circ} \mathrm{C}$ heat treatment in another UHV furnace. At this high a temperature, heavier dissolved gases (oxygen, nitrogen) diffuse out of the material and the residual resistivity ratio $R R R$ as well as the heat conductivity increase by a factor of two. To bind the oxygen diffusing out of the niobium and to prevent oxidation by the residual gas in the oven (pressure $<10^{-7} \mathrm{mbar}$ ) a thin titanium layer is evaporated on the inner and outer cavity surface, Ti being a stronger getter than Nb. The titanium layer is removed afterwards by chemical etching. A severe drawback of the $1400^{\circ} \mathrm{C}$ treatment is a considerable grain growth accompanied with a softening of the niobium. The cavities are vulnerable to plastic deformation and must be handled with great care.

\subsection{Results on cavity performance}

In this section I restrict myself to multicell cavities which are more demanding than single cell resonators. The 9-cell cavities developed for the TESLA collider are the most advanced structures concerning high gradients. Figure 48 shows the 'excitation curve' of a very good TESLA resonator. Plotted is the quality factor $Q_{0}$ as

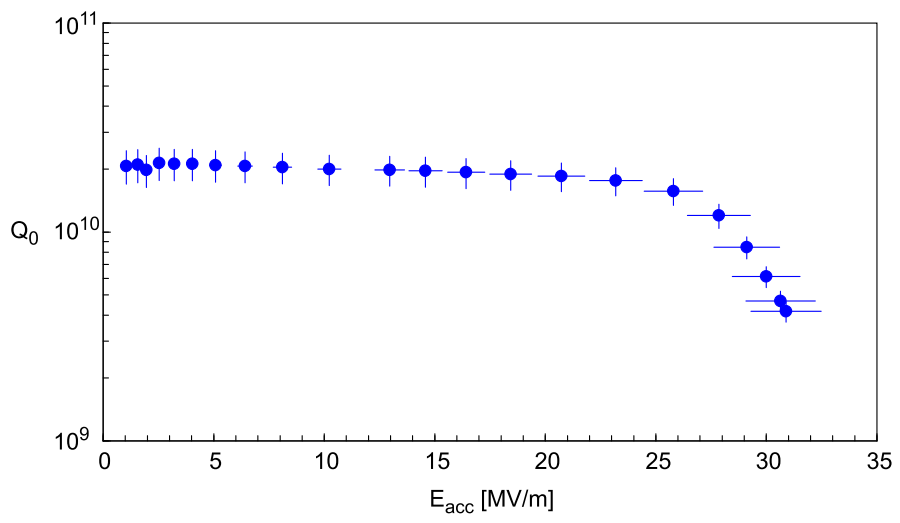

Figure 48: Excitation curve $Q_{0}=Q_{0}\left(E_{a c c}\right)$ of an excellent TESLA 9-cell cavity, cooled by superfluid helium of $2 \mathrm{~K}$.

a function of the accelerating electric field. An almost constant and high value of $2 \cdot 10^{10}$ is observed up to 25 $\mathrm{MV} / \mathrm{m}$ beyond which $Q_{0}$ decreases until the cavity becomes thermally unstable at $31 \mathrm{MV} / \mathrm{m}$. It is by no means easy to achieve such a good performance. Various treatment steps to prevent a degradation are illustrated in the next figure. A strong decrease in quality factor is usually observed if a foreign particle is sticking on the cavity surface, leading either to field emission of electrons or to local overheating in the rf field. At Cornell University a method for destroying field emitters was invented [71], called 'high power processing', which in many cases can improve the high-field capability, see Fig. 49a. Removal of field-emitting particles by high-pressure water rinsing, a technique developed at CERN [72], may dramatically improve the excitation curve (Fig. 49b). After the water rinsing the cavity must be dried and handled in a clean room to avoid a new contamination. The beneficial effect of a $1400^{\circ} \mathrm{C}$ heat treatment, first tried out at Cornell [73] and Saclay [74], is seen in Fig. 49c. The cavity performance is very poor if the titanium getter layer is not completely removed after this treatment (Fig. 49d).

By combining all mentioned steps - heat treatment at $800^{\circ} \mathrm{C}$ and $1400^{\circ} \mathrm{C}$, rinsing with ultra-pure water at high pressure, assembly in a class 100 clean room - it has been possible to achieve a dramatic improvement in the performance of industrially manufactured multicell cavities, see Fig. 50. In the most recent production of 24 nine-cell cavities the average gradient is $26 \pm 2 \mathrm{MV} / \mathrm{m}$, safely above the design gradient of $23.5 \mathrm{MV} / \mathrm{m}$ needed for a centre-of-mass energy of $500 \mathrm{GeV}$ in TESLA.

\subsection{Niobium-coated copper cavities}

Copper cavities with a thin niobium layer on the inner surface offer two advantages: a considerable cost saving and a much higher thermal conductivity of the wall and thereby better thermal stability of the cavity, see [27] for a detailed discussion. The most successful method for thin film deposition is magnetron sputtering for about 

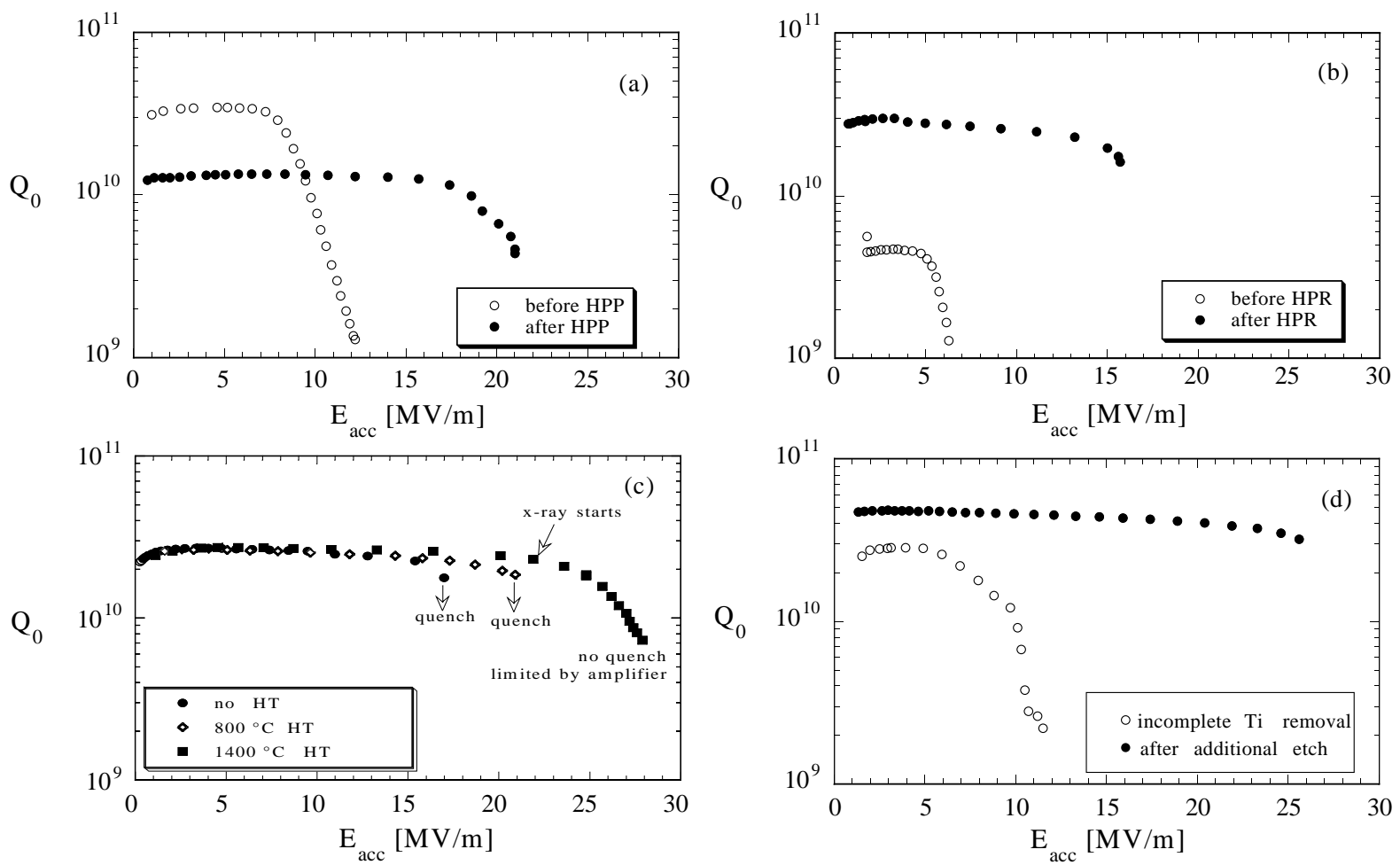

Figure 49: Impact on cavity performance of various treatment steps: (a) high power processing (HPP), (b) high-pressure water rinsing (HPR), (c) successive application of $800^{\circ} \mathrm{C}$ and $1400^{\circ} \mathrm{C}$ heat treatments $(H T)$, (d) degradation by remainders of the titanium getter layer and improvement after complete removal of this layer.

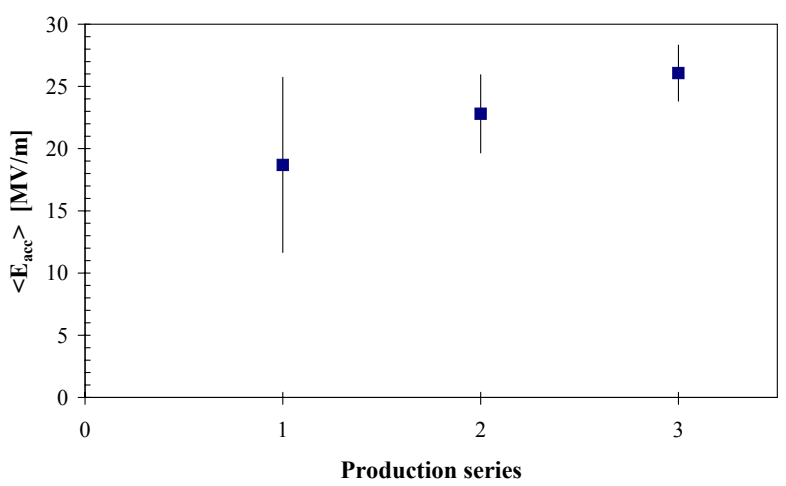

Figure 50: Average accelerating field of the industrially produced 9-cell cavities for the TESLA Test Facility (TTF) accelerator for the three production series. The error bars represent the rms spread of the distribution.
4 hours in an argon atmosphere of $1.5 \cdot 10^{-3}$ mbar and at a temperature of the clean copper substrate of 180 $200^{\circ} \mathrm{C}$. The niobium film has a thickness of a few micrometers and is usually not homogeneous but consists of rod-like grains with a diameter below $100 \mathrm{~nm}$. The residual resistivity ratio is rather low, $R R R \geq 10$. The four-cell $350 \mathrm{MHZ}$ cavities of LEP were made this way and showed excellent performance [13] at moderate gradients $(7.2 \mathrm{MV} / \mathrm{m})$, allowing to raise the centre-massenergy beyond $200 \mathrm{GeV}$ and opening the way to highly interesting investigations on pair production of $\mathrm{W}$ bosons and searches for the Higgs particle.

Considerable effort has been spent at CERN and Saclay to determine the high-field capability of single cell $1.3-1.5 \mathrm{GHz}$ copper cavities with a niobium sputter layer. Various sputter gases were tried out and many systematic studies on magnetic field depedencies were made [75], [76]. Some test results are shown in Fig. 51. For the best sputter layer $25 \mathrm{MV} / \mathrm{m}$ have been reached but with a monotonous decrease of the quality factor as a function of the accelerating field. One possible origin of this 'Q drop' are grain boundary effects in the niobium layer.

\subsection{Quest for highest gradients}

The energy upgrade of TESLA to $800 \mathrm{GeV}$ requires accelerating fields of $35 \mathrm{MV} / \mathrm{m}$, and therefore a substantial 


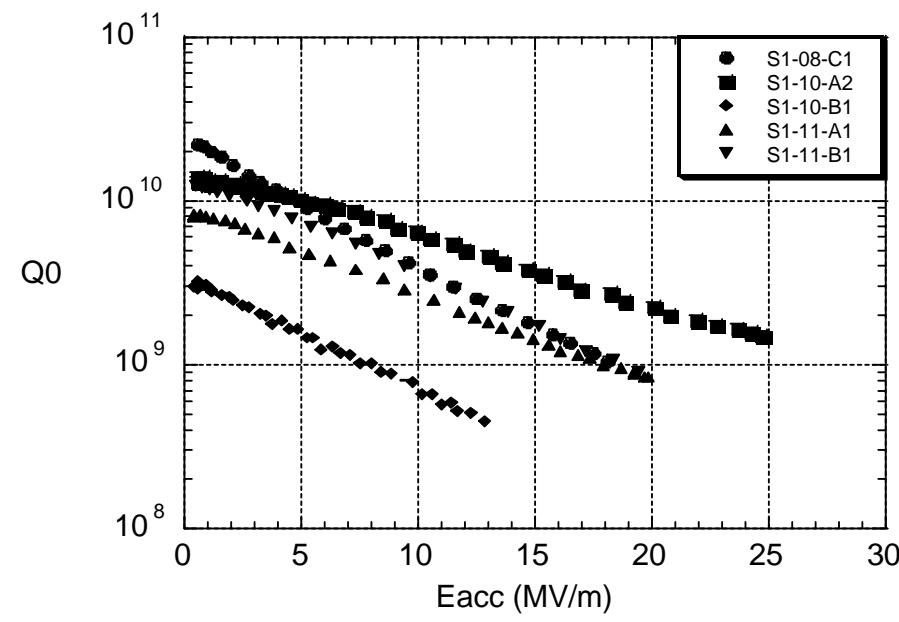

Figure 51: Quality factor as a function of accerating field for various niobium sputter coatings in a $1.3 \mathrm{GHz}$ 1-cell copper cavity.

increase in field capability is needed. The main obstacles in approaching the theoretical limit of $50 \mathrm{MV} / \mathrm{m}$ in multicell niobium cavities are foreign material contamination in the niobium and an insufficient quality and cleanliness of the inner rf surface.

\subsubsection{Quality improvement of niobium}

Niobium for microwave resonators has to be of extreme purity, for two reasons. Dissolved gases like hydrogen, oxygen and nitrogen reduce the heat conductivity at 2 $\mathrm{K}$, and contamination by normal-conducting or weakly superconducting clusters close to the rf surface may cause a premature breakdown of the superconducting state. The TTF cavities have been made from niobium with gas contents in the few ppm range and an $R R R$ of 300 . The niobium sheets were scanned with an eddy-current device to exclude sheets with foreign material contamination from the cavity production [77].

Ten 9-cell cavities were tested before and after the heat treatment at $1400^{\circ} \mathrm{C}$. The average gain in gradient was $4 \mathrm{MV} / \mathrm{m}$ which means that this heat treatment is an essential prerequisite for achieving the TESLA-500 goal, at least with the present surface preparation by chemical etching. From tests at the KEK laboratory in Tsukuba, Japan there is some evidence that the tedious and costly $1400^{\circ} \mathrm{C}$ heat treatment may not be needed in electropolished cavities (see below).

A promising new development are cavities made by hydraulic forming from a seamless tube with an inner 0.5 mm thick niobium layer and an outer $3 \mathrm{~mm}$ thick copper layer [78]. The two metals were tightly joined by explosion bonding. In a 1-cell cavity $38 \mathrm{MV} / \mathrm{m}$ were reached after chemical etching (BCP). While this technique needs still more R\&D it has the potential of a considerable savings in material cost. Another advantage is the high heat conductivity of copper allowing for a large wall thickness to suppress Lorentz-force detuning and microphonics.

\subsubsection{Electrolytic polishing}

Buffered Chemical Polishing (BCP) produces a rough niobium surface with strong etching in the grain boundaries. An gentler method to remove the damage layer is 'electropolishing' (EP). Sharp edges and burrs are smoothed out by EP and a shiny surface can be obtained, see Fig. 52. Since 1995 gradients of more than $35 \mathrm{MV} / \mathrm{m}$ have been obtained at KEK in electropolished 1-cell niobium cavities [79]. In cooperation with Saclay it has been convincingly demonstrated [80] that EP raises the accelerating field by more than $7 \mathrm{MV} / \mathrm{m}$ with respect to BCP while electropolished cavities suffer a strong degradation when they are subjected to a subsequent $\mathrm{BCP}^{14}$. In a joint R\&D program of CERN, DESY, KEK and Saclay many 1-cell cavities have been electropolished yielding gradients between 35 and $42 \mathrm{MV} / \mathrm{m}$ [81], see Fig. 52 .

The transfer of the EP technology to multicell cavities is in progress. Preliminary results are available from several 9-cell TESLA resonators which first went through the standard BCP treatment and were then electropolished by a Japanese company. The best cavity is shown in Fig. 53. By exciting the various coupled modes in the 9-cell structures it is possible to drive selected cells to higher field than the others. This way one can determine the high-field capability of individual cells ${ }^{15}$. The single-cell statistics derived from this mode analysis (Fig. 53) proves that a large number of cells exceeds $35 \mathrm{MV} / \mathrm{m}$.

Based on the test results presented in this section there is confidence that the gradient of $35 \mathrm{MV} / \mathrm{m}$ needed for $800 \mathrm{GeV}$ in TESLA can be achieved in multicell cavities within the next few years, but continuous effort will be needed to avoid field emission at these field levels. According to present knowledge, only solid niobium cavities or possibly cavities made from bonded $\mathrm{NbCu}$ tubes will be suitable for such high-field applications, while niobium-sputtered copper cavities are an economic solution for medium energy machines.

\section{Coupling of RF Power into the Cavity and Energy Transfer to the Beam}

The purpose of an accelerating cavity is to transfer radio frequency power to the particle beam. The rf cavities in a storage ring are necessarily operated in the continuous wave (cw) mode while in a high energy linear accelerator usually a pulsed operation is needed. In a normal conducting linac like SLAC the rf pulses must

\footnotetext{
${ }^{14}$ It should be mentioned that P. Kneisel of Jefferson Lab. has reached a world record of $45 \mathrm{MV} / \mathrm{m}$ in a BCP treated 1-cell cavity [82] but very high gradents were repeatedly seen only in EP treated cavities.

${ }^{15}$ There is a ambiguity in the procedure: the cells 1 and 9, 2 and 8,3 and 7,4 and 6 cannot be distinguished.
} 

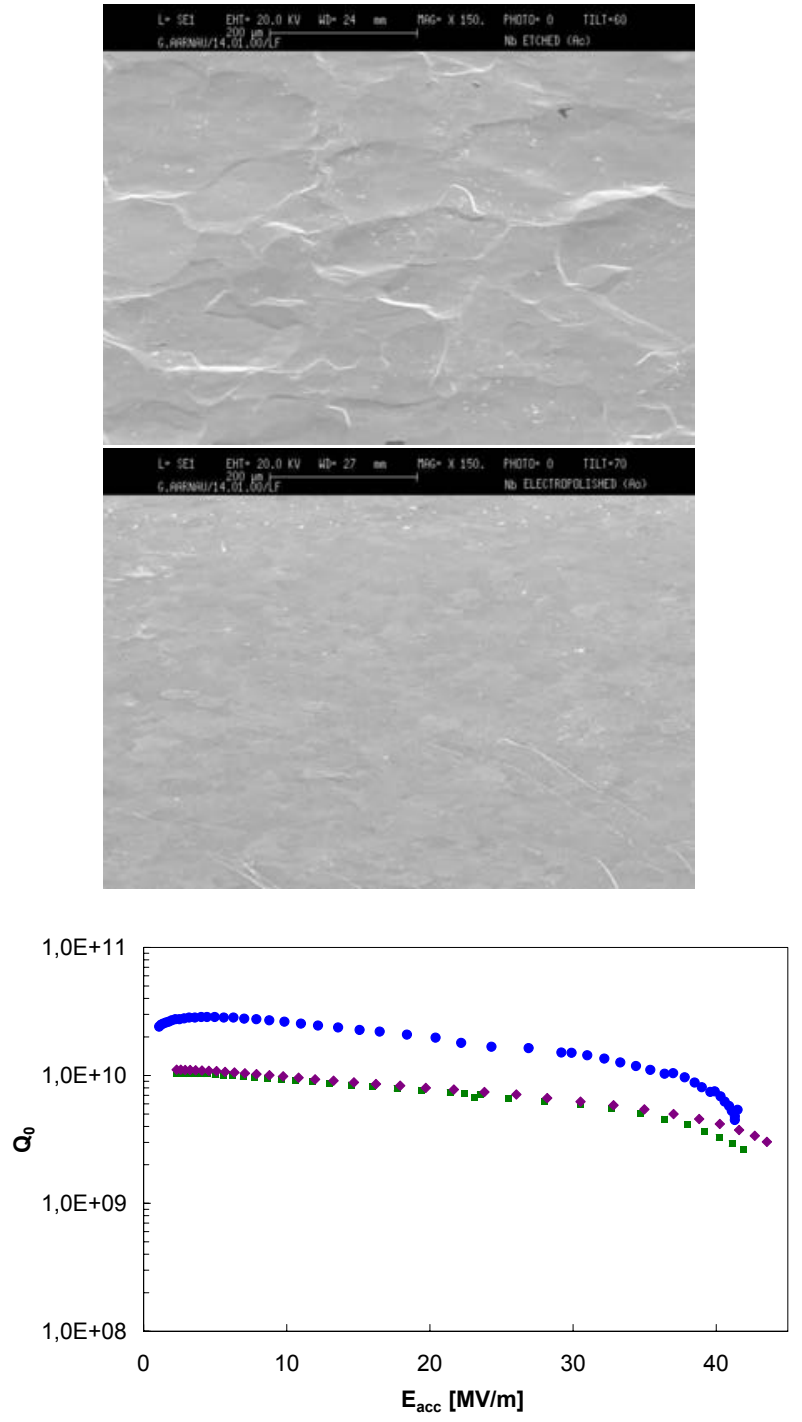

Figure 52: Top: Micrographs of a chemically etched and an electrolytically polished niobium surface. The pictures extend over $500 \mu \mathrm{m}$ horizontally. Bottom: Excitation curves of three 1-cell cavities prepared by electrolytic polishing.

be quite short, in the order of microseconds, to prevent overheating of the copper structures, in the superconducting TESLA machine the pulse duration may be a millisecond. Thereby the heat load on the refrigeration system is kept within tolerable limits. In the following I consider first the cw mode.

\subsection{Equivalent circuit diagram for a cav- ity coupled to an $\mathrm{rf}$ power source}

We represent the sc cavity by an LCR circuit (Fig. 55a) where the parallel resistor $R_{0}$, called the shunt impedance $^{16}$, is very large $\left(>10^{12} \Omega\right.$ at $\left.T=2 \mathrm{~K}\right)$. The

\footnotetext{
${ }^{16}$ The ratio $(R / Q)=R_{0} / Q_{0}=V_{a c c}^{2} /\left(2 \omega_{0} U\right)$ is an important cavity parameter which depends only on the cavity shape but not on its absolute size nor on the wall material. An alternative con-
}
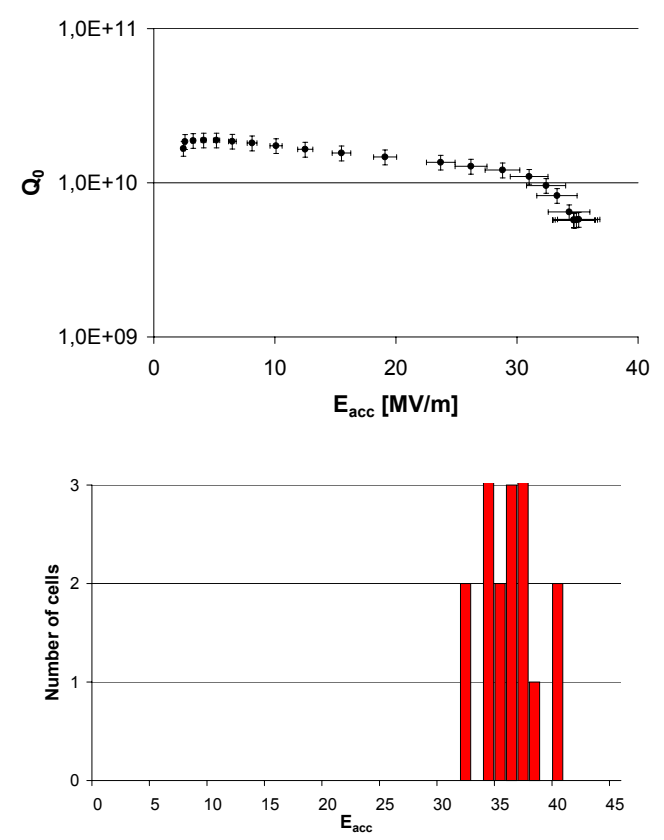

Figure 53: Results from electropolished 9-cell cavities. Top: excitation curve of the best cavity at $2 \mathrm{~K}$. Bottom: distribution of the highest acceleration fields obtained in the individual cells of two 9-cell cavities.

incorporation of the rf power source into this circuit diagram requires some care because the rf wave is guided to the cavity through a transmission line, and reflections may occur at the input to the cavity. The standard rf power sources for sc cavities used for particle acceleration are klystrons with a power rating of typically a MW, while in cavity tests without beam solid state amplifiers of $100-1000 \mathrm{~W}$ can be used. The rf wave is transported to the cavity through a transmission line (waveguide at high power, coaxial cable at low power) and coupled into the resonator by means of an input coupler. This can be a waveguide coupler or a coaxial coupler. The simplest input coupler, which is commonly used in the acceptance test of an sc cavity, is a coaxial antenna in the beam pipe section of the cavity (Fig. 54). The radius of the pipe is chosen such that its cut-off frequency is above the resonant frequency of the cavity. Hence the standing wave in the resonator is exponentially attenuated in the pipe. This means that the electric field amplitude at the tip of the coaxial antenna depends strongly on the antenna position and can be varied by several orders of magnitude when the antenna is moved longitudinally by just a few cm. Effectively, the input coupler acts as a transformer with a variable transformation ratio $1: N$ (usually $N \gg 1$ ).

It often happens that the rf wave is partly reflected at the input coupler and travels back to the rf source. Klystrons but also amplifiers may be destroyed by re-

vention is to call $R_{a}=2 R_{0}$ the shunt impedance. 


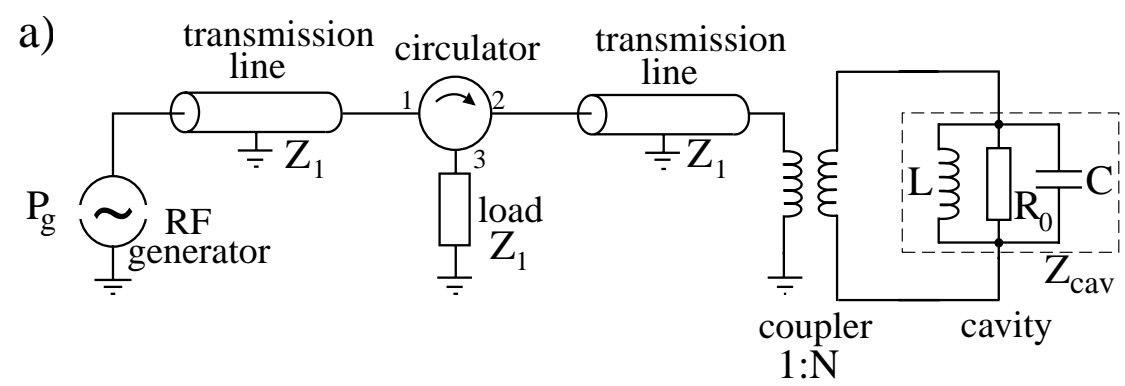

b)

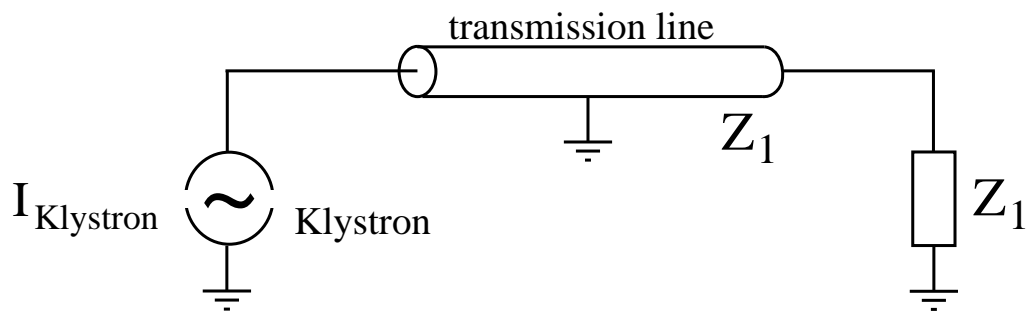

c)

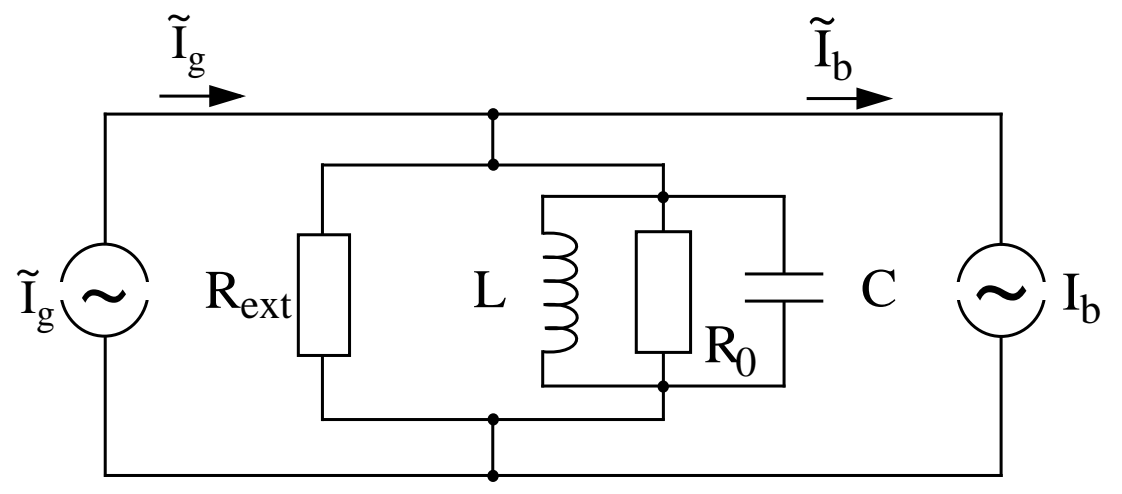

Figure 55: (a) Equivalent circuit diagram for an rf cavity with accessories: rf generator (klystron or amplifier), transmission line (wave guide or coaxial cable), circulator, input coupler, LCR circuit. (b) Circuit diagram as seen from the klystron.

(c) Circuit diagram as seen from the cavity side. The impedance of the terminated wave guide has been transformed to the cavity side and is represented by the resistor $R_{\text {ext }}=N^{2} R_{1}$. The current $I_{g}$ is given by eq. (104). Also shown is the beam current. 


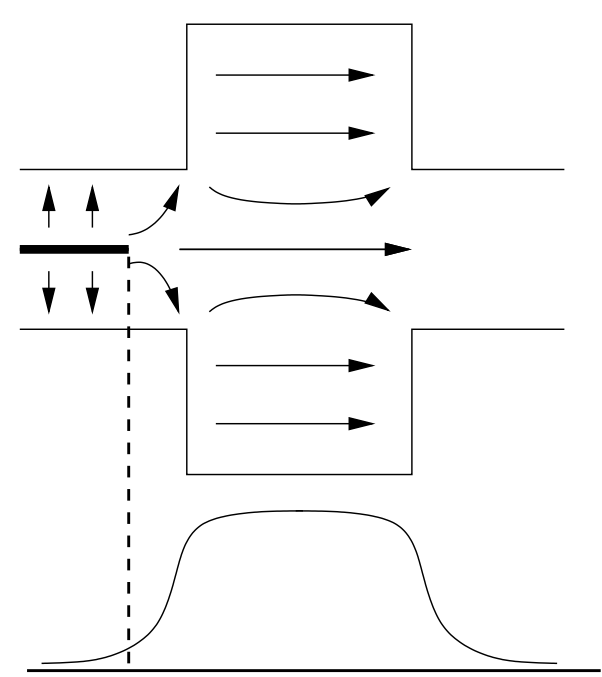

Figure 54: Schematic drawing of a pill box cavity with coaxial input coupler in the beam pipe section. The bottom curve shows the amplitude of the electric field along the cavity axis. The field decays with exponential attenuation in the beam pipe. The antenna is movable in axis direction to permit adjustment of the coupling strength.

flected rf waves. For that reason a circulator is introduced into the wave guide section between klystron and cavity, see Fig. 55a. The circulator has the following property: an rf wave entering at port 1 leaves the circulator at port 2, while a wave entering at port 2 leaves at port 3. This port is terminated with a load resistor having the characteristic impedance $Z_{1}$ of the transmission line. The wave reflected at the cavity input is thus guided into the load resistor at port 3 and completely absorbed there. Seen from the klystron, the wave guide is therefore always properly terminated, see the simplified circuit diagram of Fig. 55b. A simplified circuit diagram which does not contain transmission line components can also be drawn for the cavity side, see Fig. 55c. The wave guide with its termination $Z_{1}$ at port 3 of the circulator is transformed into the circuit as a parallel external resistance $R_{\text {ext }}=N^{2} \cdot Z_{1}$. The klystron is represented as an alternating current source.

The intrinsic quality factor of the cavity reads in terms of the lumped-circuit components

$$
Q_{0}=\frac{R_{0}}{\omega_{0} L} \quad \text { with } \quad \omega_{0}=\frac{1}{\sqrt{L C}} .
$$

The external load causes an additional damping of a free oscillation in the LC circuit. The corresponding quality factor is called the external $\mathrm{Q}$ of the cavity, $Q_{e x t}=R_{e x t} /\left(\omega_{0} L\right)$. The parallel resistors $R_{0}$ and $R_{e x t}$ can be replaced by $R_{\text {load }}=\left(1 / R_{0}+1 / R_{\text {ext }}\right)^{-1}$ which then defines the loaded quality factor

$$
Q_{\text {load }}=\frac{R_{\text {load }}}{\omega_{0} L}, \quad \frac{1}{Q_{\text {load }}}=\frac{1}{Q_{0}}+\frac{1}{Q_{\text {ext }}} .
$$

Now we consider a driven oscillation of the circuit by applying a harmonic current. For simplicity we assume that the generator frequency coincides with the cavity eigenfrequeny. Kirchhoff's rule yields

$$
C \frac{d V}{d t}+\frac{V}{R_{\text {load }}}+\frac{1}{L} \int V d t=I_{g} \cos \left(\omega_{0} t\right) .
$$

Taking the derivate with respect to time we get the equation of a driven harmonic oscillator

$$
\frac{d^{2} V}{d t^{2}}+\frac{\omega_{0}}{Q_{\text {load }}} \frac{d V}{d t}+\omega_{0}^{2} V=-\frac{I_{g} \omega_{0}}{C} \sin \left(\omega_{0} t\right)
$$

The stationary solution is

$$
V(t)=I_{g} R_{\text {load }} \cos \left(\omega_{0} t\right) .
$$

When the current is switched off we get a damped free oscillation with the time constant

$$
\tau=\frac{2 Q_{\text {load }}}{\omega_{0}}
$$

The voltage transients in pulsed operation are discussed below.

\subsection{Test of cavity without beam}

The ideal condition is that the rf generator frequency equals the cavity eigenfrequency, $\omega_{g}=\omega_{0}$, and that no power is reflected at the input coupler. This is realized if the transformed cavity impedance is equal to the characteristic impedance of the wave guide:

$$
Z_{\text {cav }} / N^{2}=R_{0} / N^{2}=Z_{1} .
$$

Note that for $\omega_{g}=\omega_{0}$ the impedance of the LCR circuit is purely real and given by $Z_{\text {cav }}=R_{0}$. Under these conditions $Q_{\text {load }}=Q_{0} / 2$, and from the time decay of the oscillation after switching off the rf generator one can easily determine the intrinsic quality factor $Q_{0}$. The generator power is fully transmitted into the cavity and dissipated in the walls, so $P_{g}=P_{d i s s}$. Measuring $P_{g}$ with a power meter allows one then to compute the stored energy $U=Q_{0} P_{d i s s} / \omega_{0}$ and the accelerating field in the cavity (in case of a pill box cavity with the help of Eqs. (84) and (91), for other cavity shapes by means of numerical codes).

It is convention to define a coupling parameter $\beta_{c}$ by

$$
\beta_{c}=\frac{R_{0}}{N^{2} Z_{1}} .
$$

Proper termination of the transmission line means $\beta_{c}=$ 1. If this is not the case, the incident $\mathrm{rf}$ generator power $P_{g}$ will be partly reflected at the input coupler, partly transmitted through the coupler and dissipated in the cavity walls:

$$
P_{\text {ref }}=\frac{\left(\beta_{c}-1\right)^{2}}{\left(\beta_{c}+1\right)^{2}} P_{g}, \quad P_{\text {trans }}=P_{\text {diss }}=\frac{4 \beta_{c}}{\left(\beta_{c}+1\right)^{2}} P_{g} .
$$


For unity coupling $\left(\beta_{c}=1\right)$ the klystron power $P_{g}$ is fully transmitted and dissipated in the resistance $R_{0}$. In the simplified circuit diagram of Fig. 55c, however, the generator current flows not only through the cavity shunt impedance $R_{0}$ but also through the external resistance $R_{\text {ext }}$. Hence the generator current $I_{g}$ is a fictitious quantity and different from the real klystron current. It can be computed for arbitrary $\beta_{c}$ from the generator power in the following way: According to Fig. $55 \mathrm{c}$ the power dissipated in $R_{0}$ is $I_{g}^{2} R_{0} /\left(2\left(\beta_{c}+1\right)^{2}\right)$. Comparing with Eq. (103) and using Eq. (102) we obtain

$$
I_{g}=2 \sqrt{\frac{2 \beta_{c} P_{g}}{R_{0}}}=\frac{2}{N} \sqrt{\frac{2 P_{g}}{Z_{1}}}=2 \frac{I_{k l y s t}}{N}
$$

which means that the fictitious current $I_{g}$ is twice the transformed klystron current $I_{k l y s t} / N$.

\subsection{Acceleration of a bunched beam}

A radio frequency cavity cannot transfer energy to a continuous beam because half of the time the field is decelerating. On the contrary, the particles must be grouped in short bunches which are synchronized with the rf wave to pass the cavity always at the desired rf phase. Respresenting the accelerating electric field by $E_{z}(t)=E_{0} \cos \left(\omega_{g} t\right)$, the nominal phase for a relativistic beam in a linear accelerator is $\phi_{0} \approx 0$, i.e. the particles are accelerated 'on crest'.

A bunch train consists of many equally spaced bunches whose repetition time $T_{\text {rep }}$ is an integer multiple of the rf period, $T_{\text {rep }}=h T_{g}=h 2 \pi / \omega_{g}$ (the integer $h$ is called the harmonic number). The rms bunch length is small, $\sigma_{b} \ll T_{g}$. In good approximation one can treat the bunch train as a periodic sequence of $\delta$-function pulses whose Fourier component at the rf frequency $\left(\omega_{g}=h \omega_{\text {rep }}\right)$ interacts with the rf wave in the cavity. This Fourier component is twice the dc component $I_{0}$ of the beam. In the lumped-circuit diagram of figure $55 \mathrm{c}$ the beam is represented by a current whose direction is chosen opposite to the generator current because the beam extracts energy from the cavity. The generator and beam currents are respectively

$$
\tilde{I}_{g} \exp \left(i \omega_{g} t\right), \quad \tilde{I}_{b} \exp \left(i \omega_{g} t\right)
$$

where $\tilde{I}_{g}$ and $\tilde{I}_{b}$ are complex phasors with $\left|\tilde{I}_{g}\right|=I_{g}$ and $\left|\tilde{I}_{b}\right|=2 I_{0}$. We consider here only the case that the generator frequeny and cavity eigenfrequency are equal, $\omega_{g}=\omega_{0}$, and that the bunches are accelerated 'on crest'. The generator-induced and beam-induced voltages in the LCR circuit are

$$
\tilde{V}_{g}=\tilde{I}_{g} \frac{R_{0}}{\beta_{c}+1}, \quad \tilde{V}_{b}=\tilde{I}_{b} \frac{R_{0}}{\beta_{c}+1}
$$

The accelerating voltage is the vectorial sum of these two voltages

$$
\tilde{V}_{a c c}=\tilde{V}_{g}+\tilde{V}_{b} .
$$

It is this quantity which changes the particle energy. For on-crest acceleration, the generator- and beam-induced voltages point in opposite direction and the net acceleration voltage is $V_{a c c}=V_{g}-V_{b}$. Using Eq. (104) to express the fictitious generator current $I_{g}$ by the generator power $P_{g}$ (i.e. the klystron power) and replacing the Fourier component of the beam current at $\omega_{g}$ by the dc component $\left(I_{b}=2 I_{0}\right)$ we get the following expression for the accelerating voltage

$$
V_{a c c}=\frac{2 \sqrt{2 \beta_{c} P_{g} R_{0}}}{\beta_{c}+1} \cdot\left(1-\frac{K}{\sqrt{\beta_{c}}}\right), \quad K=I_{0} \sqrt{\frac{R_{0}}{2 P_{g}}} .
$$

The dimensionless quantity $K$ is called the beam loading parameter. The power transferred to the beam is

$$
P_{b}=\frac{I_{b} V_{a c c}}{2}=I_{0} V_{a c c} .
$$

The efficiency of power transfer from generator (klystron) to beam is

$$
\eta_{g}=\frac{P_{b}}{P_{g}}=\frac{4 K \sqrt{\beta_{c}}}{\beta_{c}+1}\left(1-\frac{K}{\sqrt{\beta_{c}}}\right) .
$$

Finally, the power reflected at the input coupler is given by

$$
P_{\text {ref }}=P_{g}-P_{b}-P_{d i s s}=\frac{\left(\beta_{c}-1-2 K \sqrt{\beta_{c}}\right)^{2}}{\left(\beta_{c}+1\right)^{2}} \cdot P_{g} .
$$

Without beam $\left(I_{0}=0\right.$ and $\left.K=0\right)$ we recover the condition $\beta_{c}=1$ for zero power reflection at the input coupler. With beam, however, the coupling parameter of a superconducting cavity must be chosen much larger than 1 in order to achieve zero reflection. As an example we consider the 9-cell TESLA cavity at $25 \mathrm{MV} / \mathrm{m}$. The intrinsic quality factor at $T=2 \mathrm{~K}$ is $Q_{0} \approx 10^{10}$. For a bunched beam with a dc current $I_{0}=8 \mathrm{~mA}$ the generator power is $P_{g}=200 \mathrm{~kW}$. This yields a coupling parameter $\beta_{c}=3330$. The efficiency of power transfer from generator to beam is very large, $\eta_{g}=99.97 \%$. Hence almost the full generator power of $200 \mathrm{~kW}$ is transferred to the beam and only about $60 \mathrm{~W}$ are dissipated in the cavity walls, see also Table 3 . In a copper cavity, more than $50 \%$ of the generator power is wasted in heating the walls.

In a circular accelerator the nominal phase for a relativistic beam $(v \approx c)$ must be chosen on the falling slope of the rf wave $\left(0<\phi_{0}<\pi / 2\right)$ in order to achieve longitudinal focusing. In that case there is a phase angle between the generator voltage $\tilde{V}_{g}$ and the accelerating voltage $\tilde{V}_{a c c}$ which would lead to reflections at the input coupler of the cavity. It can be shown [23] that the reflection is suppressed when the generator frequency $\omega_{g}$ is detuned with respect to the cavity frequency $\omega_{0}$ by

$$
\omega_{g}-\omega_{0}=\omega_{0} \frac{I_{0} R_{0}}{Q_{0} V_{a c c}} \sin \phi_{0} .
$$



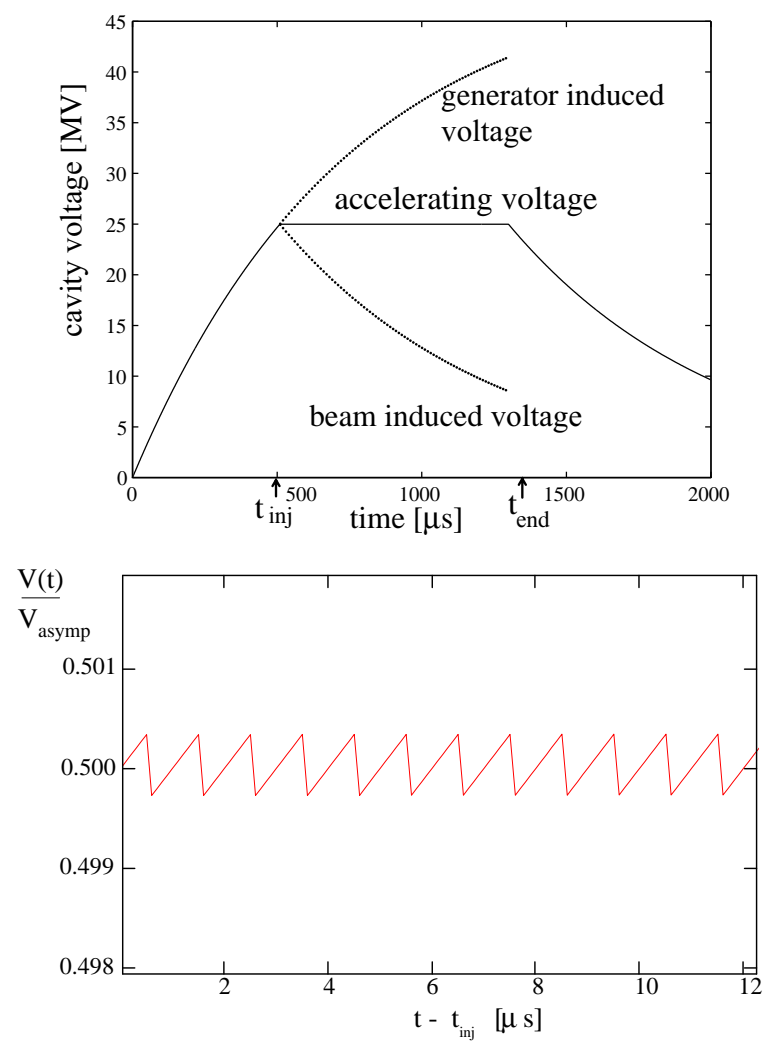

Figure 56: Top: Accelerating voltage of a TESLA cavity in pulsed-mode operation. The generator-induced voltage rises exponentially towards an asymptotic value of $50 \mathrm{MV}$. The injection of the bunched beam starts when $25 \mathrm{MV}$ have been reached. From then on the generator- and beam-induced voltages balance each other so that the accelerating voltage is maintained at the constant value of $25 \mathrm{MV}$. Bottom: Enlarged view of the plateau region. Each bunch causes a rapid decrease in cavity voltage which is replenished by the incident generator power until the arrival of the next bunch.
Table 3: Typical parameters of the $1.3 \mathrm{GHz}$ superconducting 9-cell TESLA cavity in cw operation: dc component $I_{0}$ of beam current, external quality factor $Q_{\text {ext }}$, bandwidth $\Delta f$, coupling parameter $\beta_{c}$, time constant $\tau$, klystron power $P_{g}$, dissipated power $P_{\text {diss }}$. In pulsed operation with a duty factor of 0.01 the average dissipated power is less than $1 \mathrm{~W}$.

\begin{tabular}{|c|c|c|c|c|c|c|}
\hline$I_{0}$ & $Q_{\text {ext }}$ & $\Delta f$ & $\beta_{c}$ & $\tau$ & $P_{g}$ & $P_{\text {diss }}$ \\
\hline 0 & $10^{10}$ & $0.26 \mathrm{~Hz}$ & 1 & $1.2 \mathrm{~s}$ & $60 \mathrm{~W}$ & $60 \mathrm{~W}$ \\
$8 \mathrm{~mA}$ & $3 \cdot 10^{6}$ & $433 \mathrm{~Hz}$ & 3330 & $734 \mu \mathrm{s}$ & $200 \mathrm{~kW}$ & $60 \mathrm{~W}$ \\
\hline
\end{tabular}

One gets $\omega_{g}>\omega_{0}$. This is in accordance with the Robinson criterion for avoiding collective instabilities in the synchrotron oscillations [29].

\subsection{Pulsed cavity operation}

Although the dissipated power in an sc cavity amounts to only $0.02-0.03 \%$ of the generator power, a heat deposition of about $50 \mathrm{~W}$ would require a primary electric power of $40 \mathrm{~kW}$ in the cryogenic plant to cool a 1 metre long cavity. This is far too big for a long linac. To reduce the heat load the cavities are operated in pulsed mode with a duty factor of less than $1 \%$. In pulsed operation the rf power is switched on at time $t=0$, kept constant during the interval $0 \leq t \leq t_{\text {end }}$ and then switched off. The generator-induced voltage rises exponentially towards an asymptotic value $V_{a s y m p}=I_{g} R_{0} /\left(\beta_{c}+1\right)$

$$
V_{g}(t)=V_{\text {asymp }}(1-\exp (-t / \tau)) .
$$

The injection of the bunched beam starts at a time $t_{i n j}=$ $\tau \ln 2$ when the generator-induced voltage has reached $V_{a c c}=V_{\text {asymp }} / 2$ (see Fig. 56). Each bunch of charge $q_{0}$ acts as a $\delta$-like current pulse, inducing the voltage $v_{b}=$ $-q_{0} /(2 C)$. For a periodic train of bunches these voltages add up and yield the beam-induced voltage shown in Fig. 56. The accelerating voltage is the vectorial sum of the generator- and beam-induced voltages and remains nearly constant from $t=t_{i n j}$ until $t=t_{\text {end }}$ at which time both generator power and beam are switched off. Then the cavity voltage decays according to

$$
V_{c a v}\left(t-t_{\text {end }}\right)=V_{a c c} \exp \left(-\left(t-t_{\text {end }}\right) / \tau\right) \text {. }
$$

A closer look at the plateau of the accelerating voltage reveals a sawtooth structure: each bunch causes an almost instantaneous drop of the cavity voltage which is then restored by the rising generator-induced voltage until the next bunch arrives. The beam energy spread caused by the sawtooth structure and by wake field effects is well below $0.1 \%$ for the example shown in Fig. 56 . It can be further reduced by lowering the bunch charge and increasing the number of bunches in the train correspondingly. 
Figure 56 illustrates how important it is to choose the proper cavity time constant $\tau$ for obtaining a constant accelerating voltage over the entire bunch train. Since $Q_{\text {ext }} \ll Q_{0}$ one gets in good approximation $\tau=2 Q_{\text {ext }} / \omega_{0}$ so the coupling strength of the input coupler (i.e. the winding ratio $N$ of the equivalent transformer of Fig. 55) must be properly adjusted for the given accelerating field and beam current. The TESLA input coupler is of the coaxial type and mounted on a flange at the side of the beam pipe (see Fig. 46). The central antenna is movable to permit adjustment of the coupling strength.

The short bunches excite not only the fundamental mode of the cavity but a large number of higher-order modes (HOM's). These must be damped quickly to prevent a detrimental effect on subsequent bunches. In the $500 \mathrm{MHz}$ Cornell cavity, for example, microwaveabsorbing material is installed in the beam pipe (Fig. 45), while in the TESLA cavities two HOM couplers extract the $\mathrm{HOM}$ power and guide it to damping resistors.

\section{Closing Remarks}

Superconducting magnets for high energy hadron accelerators have reached a high degree of maturity and can be considered superior to normal magnets in almost every aspect: field capability, operational costs and reliability. Proton colliders in the $\mathrm{TeV}$ regime are impossible to realize with conventional magnet technology. Superconducting cavities for particle acceleration need still research and development to arrive at a similar level of maturity, but the progress achieved in recent years gives confidence that these devices will play a major role in future accelerators.

The ideas and results outlined in this paper represent the work of many scientists at various laboratories. I am very grateful to my friends and colleagues at Brookhaven, CERN, Cornell, DESY, FNAL, INFN, Jefferson Lab., Orsay and Saclay for numerous fruitful discussions. Special thanks go to Lutz Lilje for his help in preparing the manuscript and to Matthias Liepe for valuable comments.

\section{A Gibbs Free Energy}

To illustrate the purpose of the free energy I consider first an ideal gas. The internal energy is the sum of the kinetic energies of all atoms

$$
U=\sum_{i=1}^{N} \frac{m}{2} v_{i}^{2}=\frac{3}{2} N k_{B} T
$$

and depends only on temperature but not on volume. The first law of thermodynamics describes energy conservation:

$$
d U=\delta Q+\delta W
$$

The internal energy increases either by adding heat $\delta Q$ or mechanical work $\delta W=-p d V$ to the gas. For a reversible process one has $\delta Q=T d S$ where $S$ is the entropy. Now consider an isothermal expansion of the gas. Thereby the gas transforms heat into mechanical work:

$$
d U=0 \quad \text { for } T=\text { const } \Rightarrow \delta Q=-\delta W=p d V .
$$

The gas extracts heat from a reservoir and transforms it into mechanical work. However its internal energy does not change, hence $U$ is not an adequate variable to describe the process. What is the correct energy variable? We will see that this is the Helmholtz free energy, given by

$F=U-T S \Rightarrow d F=d U-S d T-T d S=\delta W-S d T$.

For an isothermal expansion $(d T=0)$ we get $d F=\delta W$, i.e. $-d F=p d V$ : the work produced by the gas is identical to the reduction of its free energy.

Now we consider a magnetic material of permeability $\mu$ inside a coil which generates a field $H$. The magnetization is $\vec{M}=(\mu-1) \vec{H}$. Its potential energy (per unit volume) in the magnetic field is

$$
E_{p o t}=-\mu_{0} \vec{M} \cdot \vec{H} .
$$

If the magnetization changes by $d \vec{M}$ the work is $\delta W=$ $\mu_{0} d \vec{M} \cdot \vec{H}$. Defining again the Helmholtz free energy by eq. (118) we get by analogy with the ideal gas $d F=\delta W$, hence $F$ can in fact be used to describe the thermodynamics of magnetic materials in magnetic fields. One drawback is, however, that the magnetization of a substance cannot be directly varied by the experimenter. What can be varied at will is the magnetic field $H$, namely by choosing the coil current. For this reason another energy function is more appropriate, the Gibbs free energy

$$
G=F-\mu_{0} \vec{M} \cdot \vec{H}=U-T S-\mu_{0} \vec{M} \cdot \vec{H} .
$$

For an isothermal process we get $d G=-\mu_{0} \vec{M} \cdot d \vec{H}$. Let us apply this to a superconductor in the Meissner phase. Then $\mu=0$ and $\vec{M}=-\vec{H}$ from which follows $d G_{\text {sup }}=\mu_{0} M(H) d H=\frac{\mu_{0}}{2} d\left(H^{2}\right)$ and therefore

$$
G_{\text {sup }}(H)=G_{\text {sup }}(0)+\frac{\mu_{0}}{2} H^{2} .
$$

This equation is used in sect. 2.2. 


\section{References}

[1] Review of Particle Properties, Europ. Phys. Journ. C15 (2000)

[2] Helen Edwards, The Tevatron Energy Doubler: A Superconducting Accelerator, Ann. Rev. Nucl. Part. Science, 1985

[3] B.H. Wiik, HERA : machine and experiment, Proc. 24th Int. Conf. on High-Energy Physics, Munich, Germany, 1988, p. 404

[4] Design study of the Large Hadron Collider (LHC) : a multiparticle collider in the LEP tunnel, CERN report91-03 (1991)

[5] RHIC Design Manual, Brookhaven National Laboratory BNL 1993, http://www.agsrhichome.bnl.gov/NTshare/rhicdm

[6] C. Adolphsen et al., SLAC Report 474 (1996)

[7] JLC Design Study, KEK Report 97-1 (1997)

[8] G. Guignard et al., CERN Report 2000-008 (2000)

[9] B.H. Wiik, Part. Acc. 62 (1998) 563

[10] TESLA, Technical Design Report, The Accelerator, Eds. R. Brinkmann, K. Flöttmann, J. Rossbach, P. Schmüser, N. Walker, H. Weise, DESY report 2001-23 (2001)

[11] G.A. Krafft, Status of the Continuous Electron Beam Accelerator Facility, Proc 1994 Linear Accel. Conf., 9 (1994)

[12] International Linear Collider Technical Review Committee Report II (2002), ed. G.A. Loew, to be published

[13] P. Brown et al., CERN report SL-2002-004 (HRF) and Proc. 10th Workshop on RF Superconductivity, Tsukuba, Japan, 2001, SA001

[14] H. Brechna, Superconducting Magnet Systems, Springer, Berlin 1973

[15] M.N. Wilson, Superconducting Magnets, Oxford University Press, 1997

[16] K.-H. Mess, P. Schmüser, S. Wolff, Superconducting Accelerator Magnets, World Scientific, Singapore, 1996

[17] A.V. Tollestrup, IEEE Trans. NS-28 (1981) 3198

[18] P. Schmüser, Rep. Progr. Phys. 54 (1991) 683

[19] S. Wolff, in: M. Month, M. Dienes (Eds.), The Physics of Particle Accelerators, AIP Conf. Proc. 249 (1992) 1159

[20] H. Desportes, Cryogenics 34, ICEC Suppl. (1994) 47

[21] A. Greene, P.C. Dent and C. Hallquist, Superconductor Industry Vol. 8 (1995) 16, Rodman Publ., New Jersey

[22] R. Perin, Part. Acc. 28 (1990) 147 and IEEE Trans. ASC-5 (1995) 189
[23] H. Padamsee, J. Knobloch and T. Hays, RF Superconductivity for Accelerators, John Wiley, New York 1998.

[24] H. Piel in: Proc. of the 1988 CERN Accelerator School Superconductivity in Particle Accelerators, CERN report 89-04 (1989)

[25] W. Weingarten in: Proc. of the 1995 CERN Accelerator School Superconductivity in Particle Accelerators, CERN report 96-03 (1996)

[26] D. Proch, Rep. Prog. Phys. 61 (1999) 1

[27] H. Padamsee, Supercond. Sci. Technol. 14 (2001) R28R51

[28] Proceedings of the 1988 and 1995 CERN Accelerator Schools Superconductivity in Particle Accelerators, ed. S. Turner, CERN reports 89-04, 96-03

[29] A. Chao, M. Tigner (Ed.), Handbook of Accelerator Physics and Technology, World Scientific, Singapore, 1999

[30] D.C. Larbalestier and P.J. Lee, Proc. PAC99, New York (1999) 177

[31] W. Buckel, Supraleitung, VCH Verlagsgesellschaft, Weinheim 1990

[32] D.R. Tilley and J. Tilley, Superfluidity and Superconductivity, Institute of Physics Publishing Ltd, Bristol 1990

[33] Y.B. Kim, C.F. Hempstead and A.R. Strnad, Phys. Rev. A 139 (1965) 1163

[34] E.W. Collings et al., Adv. Cryog. Eng. 36 (1990) 169

[35] Z. J. Stekly and J. L. Zar, IEEE Trans. NS-12 (1965) 367

[36] D.E. Baynham et al., IEEE Trans. MAG-17 (1981) 732

[37] J. G. Cottingham, Brookhaven report BNL-16816 (1971)

[38] A.P. Verweij and H.H.J. ten Kate, IEEE Trans. ASC-5 (1995) 404

[39] C.P. Bean, Phys. Rev. Lett. 8 (1962) 250 and Rev. Mod. Phys. (1964) 31

[40] J.-L. Duchateau, Department Saturne internal report SEDAP/72-109 (1972)

[41] M.A. Green, IEEE Trans. NS-18 (1971) 664

[42] C. Völlinger, M. Aleksa, and S. Russenschuck, Phys. Rev. STAB, Acc. and Beams 3 (2000) 122402

[43] H. Brück, R. Meinke, F. Müller, P. Schmüser, Z. Physik C44 (1989) 385

[44] D.A. Finley et al., Proc. PAC87, Washington D.C. 1987, p. 151

[45] Y.B. Kim, C.F. Hempstead and A.R. Strnad, Phys. Rev. Lett. 9 (1962) 306 
[46] P.W. Anderson, Phys. Rev. Lett. 9 (1962) 309

[47] H. Brück et al., Proc. 11th Int. Conf. Magn. Techn. MT11, Tsukuba, Japan (1989) 141

[48] M. Halemeyer, P. Schmüser et al., IEEE Trans. ASC-3 (1993) 168

[49] L. Krempaski and C. Schmidt, Appl. Phys. Lett. 66 (1995) 1545, and J. Appl. Phys. 78 (1995) 5800

[50] H. Brück, P.D. Gall et al., Proc. PAC91, San Francisco 1991, p. 2149

[51] A.K. Ghosh, K.E. Robins, W.B. Sampson, Proc. PAC93, Washington D.C., 1993

[52] G.H. Morgan, J. Appl. Phys. 44 (1973) 3319

[53] A. Devred, T. Ogitsu, KEK report 94-156 (1994)

[54] A. Akhmetov, A. Devred, T. Ogitsu, J. Appl. Phys. 75 (1994) 3176

[55] A.P. Verweij, PhD thesis, Twente University 1995

[56] J.P. Ozelis et al., IEEE Trans. ASC-3 (1993) 678

[57] R. Shafer, IEEE Trans. MAG-17 (1981) 722

[58] R. Shafer, K.M. Smedley, Proc. Int. Conf. High Energy Acc., Hamburg 1992, World Scientific 1993, p. 298 and 629

[59] G. Müller, Proc. 3rd Workshop on RF Superconductivity, ed. K.W. Shepard, Argonne, USA (1988), p. 331

[60] B. Bonin, CERN Accelerator School Superconductivity in Particle Accelerators, CERN 96-03, ed. S. Turner, Hamburg (1995)

[61] F. Palmer, Proc. 3rd Workshop on RF Superconductivity, K. Shephard (ed.), Argonne Natl. Lab. 1988, p. 309

[62] J. Halbritter, Z. Physik 238 (1970) 466

[63] C. Benvenuti et al., Proc. PAC91, San Francisco (1991), p. 1023.

[64] A. Boucheffa et al., Proc. 7th Workshop on RF Superconductivity, ed. B. Bonin, Gif-sur-Yvette, France (1995), p. 659

[65] T. Schilcher, TESLA-Report, TESLA 95-12, DESY (1995)

[66] T. Hays, H. Padamsee, Proc. 8th Workshop on RF Superconductivity, V. Palmieri, A. Lombardi (eds.), Abano Terme Italy 1997, p. 789

[67] B. Aune et al., Phys. Rev. Spec. Top. Acc. Beams PRSTAB 3 (2000) 092001

[68] H. Padamsee, Proc. 5th Workshop on Superconductivity, Hamburg, Germany (1991), p. 904

[69] R.H. Fowler and L. Nordheim, Proc. of the Roy. Soc. A 119, 173 and Math. Phys. Sci. 119 (1928) 173.

[70] M. Liepe, Proc. Linac 2000 Conf. (2000) p. 678
[71] J. Graber, Nucl. Instrum. and Meth. A 350 (1994) 572, C. Crawford et al., Part. Acc. 49 (1995) 1

[72] P. Bernard et al., Proc. 1992 Europ. Part. Accel. Conf., ed. H. Henke et al., Editions Frontieres (1992), p. 1269

[73] H. Padamsee et al., Proc. 4th Workshop on RF Superconductivity, ed. Y. Kojima, Tsukuba, Japan (1990)

[74] H. Safa et al., Proc. 7th Workshop on RF Superconductivity, ed. B. Bonin, Gif-sur-Yvette, France (1995), p. 649

[75] V. Arbet-Engels et al., Nucl. Instr. and Meth. in Phys. Res. A 463 (2001) 1

[76] P. Bosland et al., IEEE Trans. ASC-98, Vol. 9, No. 2 (1999)

[77] W. Singer et al., Proc. 8th Workshop on RF Superconductivity, Eds. V. Palmieri, A. Lombardi, Abano Terme, Italy (1999), p. 850

[78] W. Singer et al., Proc. 10th Workshop on RF Superconductivity, Tsukuba, Japan, 2001, FA009.

[79] M. Ono et al., Proc. 8th Workshop on RF Superconductivity, Eds. V. Palmieri and A. Lombardi, Abano Terme, Italy (1997), p. 472

[80] E. Kako, Proc. 9th Workshop on RF Superconductivity, Santa Fe, USA (1999), TUP011, p. 179

[81] L. Lilje et al., Proc. 9th Workshop on RF Superconductivity, Santa Fe, USA (1999), TUA001, p. 74

[82] P. Kneisel, R. Röth and H.-G. Kürschner, Proc. 7th Workshop on RF Superconductivity, ed. B. Bonin, 1995, Gif-sur-Yvette, France, p. 449. 